\title{
The Application of Cryogenic Laser Physics to the Development of High Average Power Ultra-Short Pulse Lasers
}

\author{
David C. Brown ${ }^{1, *}$, Sten Tornegård ${ }^{1}$, Joseph Kolis ${ }^{2}$, Colin McMillen ${ }^{2}$, Cheryl Moore ${ }^{1,2}$, \\ Liurukara Sanjeewa ${ }^{2}$ and Christopher Hancock ${ }^{1}$
}

Received: 21 November 2015; Accepted: 5 January 2016; Published: 20 January 2016

Academic Editor: Malte C. Kaluza

1 Snake Creek Lasers, LLC, 26741 State Route 267, Friendsville, PA 18818, USA; stornegard@snakecreeklasers.com (S.T.); cherylm@clemson.edu (C.M.);

chancock@snakecreeklasers.com (C.H.)

2 Department of Chemistry, Clemson University, Clemson, SC 29634-0973, USA; kjoseph@clemson.edu (J.K.); cmcmill@g.clemson.edu (C.M.); lsanjee@g.clemson.edu (L.S.)

* Correspondence: dbrown@snakecreeklasers.com; Tex: +570-553-1122; Fax: +570-553-1139

\begin{abstract}
Ultrafast laser physics continues to advance at a rapid pace, driven primarily by the development of more powerful and sophisticated diode-pumping sources, the development of new laser materials, and new laser and amplification approaches such as optical parametric chirped-pulse amplification. The rapid development of high average power cryogenic laser sources seems likely to play a crucial role in realizing the long-sought goal of powerful ultrafast sources that offer concomitant high peak and average powers. In this paper, we review the optical, thermal, thermo-optic and laser parameters important to cryogenic laser technology, recently achieved laser and laser materials progress, the progression of cryogenic laser technology, discuss the importance of cryogenic laser technology in ultrafast laser science, and what advances are likely to be achieved in the near-future.
\end{abstract}

Keywords: ultrafast lasers; diode-pumped; cryogenic lasers; high-peak-power lasers; high-average-power lasers

\section{Introduction and History of Cryogenic Lasers}

The history of the development of cryogenic lasers may roughly be divided into two distinct eras, which we refer to as the historical and the modern. The historical era covers the early use of cryogenic technology to effect the demonstration of new solid-state lasers, and was focused on the spectroscopic and lasing properties using flashlamp optical pumping, while in the modern era, the advent of diode optical pumping and the increased understanding of the physical properties of laser crystals has led to a comprehensive approach to the development of cryogenic lasers. The comprehensive approach embraces not just the spectroscopic and lasing properties, but includes all of the physical properties needed to successfully design and demonstrate advanced cryogenic laser devices. Such properties include mechanical, thermal, thermo-optic and optical properties such as the index of refraction as well as the crystal dispersion properties. Other defining features of the modern era are the maturation of open and closed cycle cryogenic cooling methods and the rapid development and availability of powerful diode laser sources for pumping solid-state lasers.

In 1991, the first demonstration of a liquid nitrogen $\left(\mathrm{LN}_{2}\right)$ cooled diode-pumped $\mathrm{Yb}$ :YAG laser was reported by Lacovara et al. [1], and we rather arbitrarily define the year of publication of that paper as the start of the modern era, because it combined cryogenic-cooling with a diode-pumped Yb:YAG laser crystal. Some may argue or imply that the development of cryogenic lasers in the modern 
era is really nothing new, simply because cryogenically-cooled lasers were first demonstrated in the early years of the development of solid-state lasers and there exist a number of early cryogenic laser patents. Such arguments however ignore the reality that during the historical period all cryogenic lasers were flashlamp-pumped with concomitant large heat generation in the solid-state laser crystal, significantly reducing the effectiveness of cryogenic-cooling. In addition, the modern era has as its goal the demonstration of cryogenic laser technology whose average power scaling is well-understood and is firmly grounded in the detailed measured properties of a number of key laser host physical parameters. The long-sought goal of laser physicists, engineers, and designers to produce near diffraction-limited output lasers while operating at very high average powers and efficiency, and whose output parameters are invariant to average power, is now being realized. It is important to emphasize and acknowledge the countless individual contributors whom have made this goal a reality, spanning both the historical and modern periods of together almost seven decades. It is gratifying that the promise of cryogenic lasers, articulated in an article one decade ago [2] and another 8 years ago [3], is beginning to be realized. Yet it is also exciting to contemplate that we have only just begun to realize the vast potential of cryogenic lasers, and that could not be more true than in the nascent field of cryogenic ultrafast lasers.

This article discusses cryogenic laser technology, with a particular emphasis on the application of that technology to the development of ultrafast lasers. The intersection of cryogenic and ultrafast laser technologies will undoubtedly provide powerful new laser sources capable of producing extraordinary associative peak and average powers in the near future. We refer to these new lasers as HAPP (High Average and Peak Power) lasers.

\subsection{The Historical Era}

Good reviews of the use of cryogenic-cooling in the development of solid-state lasers have been presented by members of our group [2], the Massachusetts Institute of Technology (MIT) Lincoln Laboratory laser group [3], and by the laser group at Q-Peak [4]. Of particular note are the works of Keyes and Quist [5] whom used a GaAs diode laser to pump a $\mathrm{U}^{3+}: \mathrm{CaF}_{2}$ laser (the first diode-pumped laser), wherein both the diode laser and the laser crystal were cryogenically-cooled, the pioneering Ti: $\mathrm{Al}_{2} \mathrm{O}_{3}$ work of Moulton [6] which demonstrated increased power output using a liquid nitrogen cooled (at $77 \mathrm{~K}$ ) crystal, an effect that was attributed to the increased $\mathrm{Al}_{2} \mathrm{O}_{3}$ thermal conductivity, and the development of a number of tunable laser materials by Moulton [7-10]. The pioneering work of Schulz and Henion [11] whom demonstrated $350 \mathrm{~W}$ of output power from a liquid-nitrogen cooled Ti: $\mathrm{Al}_{2} \mathrm{O}_{3}$ crystal, over 200 times what could be obtained at room temperature, is particularly noteworthy since those researchers were the first to our knowledge to carefully examine the crucial role materials properties such as thermal conductivity, thermal expansion coefficient, and the thermo-optic coefficient $(\mathrm{d} n / \mathrm{d} T)$ play in substantially reducing thermal aberrations and stresses as temperature is lowered from room temperature to $77 \mathrm{~K}$ in sapphire. An evaluation of the data available at the time, summarized in Table I of [11], revealed that the thermal conductivity of $\mathrm{Al}_{2} \mathrm{O}_{3}$ increased from 0.33 to $10 \mathrm{~W} /(\mathrm{cm} \cdot \mathrm{K})$ as temperature was lowered from $300 \mathrm{~K}$ to $77 \mathrm{~K}$, a greater than 30 times improvement. The thermo-optic coefficient decreased from $12.8 \times 10^{-6} / \mathrm{K}$ at $300 \mathrm{~K}$ to $1.9 \times 10^{-6} / \mathrm{K}$, a factor of 6.7 , while the thermal expansion coefficient decreased from $5 \times 10^{-6} / \mathrm{K}$ at $300 \mathrm{~K}$ to $0.34 \times 10^{-6} / \mathrm{K}$ at $77 \mathrm{~K}$, a factor of 14.7. In cooling the Ti: $\mathrm{Al}_{2} \mathrm{O}_{3}$ laser to $77 \mathrm{~K}$, the authors were able to demonstrate about a 200 times decrease in the thermo-optical refractive index changes. Other early references of note include the demonstration of a tunable phonon-terminated $\mathrm{Ni}^{2+}: \mathrm{MgF}_{2}$ laser by Johnson, Guggenheim, and Thomas [12] at Bell Telephone Laboratories in 1963, and the development of a compact liquid nitrogen cooled system that produced $\mathrm{CW}$ emission from $\mathrm{Nd}^{3+}$ :YAG laser crystal as well as an ABC-YAG laser crystal [13]. In Europe, an early cryogenic Ho:YAG laser was demonstrated in 1975; an output of $50 \mathrm{~W}$ was achieved with liquid nitrogen cooling [14].

In addition to laser demonstrations, cryogenic cooling has previously been used in spectroscopic solid-state laser crystal investigations to help elucidate the physics, energy level structure, and 
determine energy level assignments, for many rare-earth and transition metal activator ions. The laser crystal books by Kaminskii $[15,16]$ are particularly noteworthy, documenting many of the Stark level structures and energy assignments in use today at room temperature, $77 \mathrm{~K}$, and in some cases down to liquid He temperature at about $4 \mathrm{~K}$. Those publications are very useful resources that include historical spectroscopic and laser investigations from around the world, including many from Russia and former Soviet Republics whose many contributions were not fully acknowledged until after the dissolution of the Soviet Union in 1991.

Early cryogenic laser patents were issued in the United States to Bowness [17] for a liquid nitrogen cooled ruby laser, and to McMahon [18] for a cryogenically-cooled ruby laser. Other early cryogenic work in the United States was performed at Sanders Associates (now BAE Systems, Inc., Arlington, VA, USA), in Nashua, NH.

\subsection{The Modern Era}

The genesis of our cryogenic laser program was the publication of the Schulz and Henion paper in 1991 [11]. Starting in 1995, we investigated whether or not the favorable laser and thermal effects exhibited by Ti: $\mathrm{Al}_{2} \mathrm{O}_{3}$ might not be operative in other laser crystals as well, and in particular for the well-developed laser material $\mathrm{Y}_{3} \mathrm{Al}_{5} \mathrm{O}_{12}$. After a thorough literature search, we found enough high quality previously published thermal conductivity and thermal expansion coefficient data to begin to at first examine thermal and stress induced changes in undoped YAG, with a view towards understanding the detailed ramifications of lowering the temperature from room temperature to $77 \mathrm{~K}$. That work led to the award and completion of a Phase I Small Business Innovation Research (SBIR) contract in 1997, with the Final Report published in 1998 [19]. Work completed prior to and during that contract led to three publications [20-22] during 1997 and 1998 in which the average power scaling behavior and the reduction of thermally-induced aberrations were thoroughly explored and quantified for the first time using a unified theoretical framework. In [20], we were the first group to use a finite element thermal and stress analysis code with continuously variable materials parameters (thermal conductivity, thermal expansion coefficient, and the elastic parameters Young's modulus and Poisson's ratio, as a function of temperature), to simulate the expected performance of solid-state YAG amplifiers at $77 \mathrm{~K}$. The continuously variable thermal and elastic parameters were generated by good fits to the data existing at the time. Applying the theory to Nd:YAG and Yb:YAG lasers, we showed that the previous results obtained for Ti: $\mathrm{Al}_{2} \mathrm{O}_{3}$ lasers [11] could indeed be emulated using other oxide laser hosts such as YAG, and that very favorable, but non-linear, scaling to very high average powers could be obtained by operating YAG at $77 \mathrm{~K}$. We also showed for the first time that when a continuously varying thermal conductivity is employed in the finite-element simulations, non-quadratic temperature profiles are obtained.

In addition to exploring the scaling properties of cryogenically-cooled YAG lasers, in [20] we also explored how thermally-induced aberrations vary with temperature, and showed that without taking the thermo-optic effect into account, approximately a factor of 10 improvement could take place by operating at $77 \mathrm{~K}$, due to the deduction of the thermal profile by an increased thermal conductivity, and by the near elimination of stress-optic effects. We also lamented the apparent lack of thermo-optic $(\beta=\mathrm{d} n / \mathrm{d} T)$ data for YAG and other optical materials as temperature is lowered from 300 to $77 \mathrm{~K}$, but pointed out that the expected trend of $\beta$ towards zero as temperature is lowered would further increase the reduction in thermo-optic effects in cryogenic lasers. The basic principles of low thermo-optic effects in the host YAG were correctly predicted in this article, confirming our previous conjecture that YAG and other oxide laser host crystals would behave very much like the oxide crystal Ti: $\mathrm{Al}_{2} \mathrm{O}_{3}$ demonstrated experimentally previously [11].

The publication of the first accurate YAG thermo-optic coefficients by Fan and Daneu in 1998 [23] enabled us to further extend our results to include $\beta$, and in the following papers in 1998 [21,22], we presented the first comprehensive theoretical description of the expected reduced thermal distortion at $77 \mathrm{~K}$ in YAG for rod amplifiers, and nonlinear thermal and stress effects in YAG slabs, as well as the 
scaling behavior. The results presented in [20-22] assumed an undoped host YAG thermal conductivity variation with temperature, and did not include a description of the reduction in thermal conductivity in certain materials as the lasing ion dopant density increases. This effect will be further explored later in this article. During 1998 Fan et al. [24] presented the first experimental confirmation, using a $940 \mathrm{~nm}$ diode-pumped Yb:YAG crystal, that cooling the Yb:YAG crystal to $77 \mathrm{~K}$ resulted in a large reduction in thermo-optic effects, as well as a CW power output of $40 \mathrm{~W}$. Further confirmation of the large reduction in thermal effects, in Nd:YAG, was provided in 2004 by Glur et al. [25].

In 2005 we published an article further confirming the trends discussed in the aforementioned [2]. To provide confirmation that the same general behavior of the thermal conductivity, thermal expansion coefficient, and $\mathrm{d} n / \mathrm{d} T$ could be expected in other crystals, we showed additional literature data. For the thermal conductivity, we showed data for $\mathrm{LiF}, \mathrm{MgO}, \mathrm{Al}_{2} \mathrm{O}_{3}, \mathrm{C}, \mathrm{YbAG}, \mathrm{LuAG}$, and YAG, all of which displayed significant increases at temperatures were lowered to $77 \mathrm{~K}$ and below. As expected, the behavior of the thermal conductivities for undoped crystals is dominated by the specific heat $C_{\mathrm{V}}$, which theoretically increases as approximately the inverse of the absolute temperature between 300 and $77 \mathrm{~K}$. It is not surprising that fluoride and oxide hosts display the same general trend. For the thermal expansion data, we plotted data for seven different crystals, including the recently published data of the MIT Lincoln Lab group [23,26], all of which could be seen converging towards a value close to zero at low temperatures. For the thermo-optic coefficient $\mathrm{d} n / \mathrm{d} T$, we used the MIT data as well as literature data found for both axes of $\mathrm{Al}_{2} \mathrm{O}_{3}, \mathrm{ZnSe}, \mathrm{Ge}$, and $\mathrm{Si}$. In all cases substantial decreases in $\mathrm{d} n / \mathrm{d} T$ were found as temperature is lowered. These results showed conclusively that the same trends for the thermal conductivity, thermal expansion coefficient, and $\mathrm{d} n / \mathrm{d} T$ were apparent across a wide sampling of crystals. In addition to examining critical laser materials parameters, our paper also presented for preliminary cryogenic absorption cross-section data for Yb:YAG, measured between 300 and $77 \mathrm{~K}$, and later presented in a more detailed publication [27]. Previously, absorption coefficients for Yb:YAG at 300 and $77 \mathrm{~K}$ were presented in [24]. A significant finding of that work was that at $77 \mathrm{~K}$ the $940 \mathrm{~nm}$ pump band doubled in intensity and narrowed, but remained broad enough for pumping with conventional diode pump sources. For quasi-three-level Yb materials like Yb:YAG that display finite ground-state laser absorption at room temperature, cryogenic-cooling also results in 4-level performance at $77 \mathrm{~K}$, reducing the pump density needed to achieve transparency from a significant value to almost zero. We point out here that the large increase in the stimulated-emission cross-section from a value of about $1.8 \times 10^{-20} \mathrm{~cm}^{2}$ at room temperature [1] to $1.1 \times 10^{-19} \mathrm{~cm}^{2}$ at $80 \mathrm{~K}$ [3] by approximately a factor of 6 reduces the saturation fluence and intensity by the same factor, thus positively affecting the extraction and overall efficiencies that may be achieved. To achieve good amplifier extraction efficiency the incident amplifier fluence must take a value of at least 2 times the saturation fluence, and for Gaussian beams must be even higher [28]. A much smaller saturation fluence enhances the likelihood of being able to operate below the damage threshold fluence while at the same time achieving efficient extraction efficiency.

Also in 2005, an impressive paper that for the first time measured the detailed thermo-optic properties of a large sampling of laser crystals from 300 to $80 \mathrm{~K}\left(\mathrm{Y}_{3} \mathrm{Al}_{5} \mathrm{O}_{12}(\mathrm{YAG}), \mathrm{Lu}_{3} \mathrm{Al}_{5} \mathrm{O}_{12}\right.$ (LuAG), $\mathrm{YAlO}_{3}(\mathrm{YALO}), \mathrm{LiYF}_{4}(\mathrm{YLF}), \mathrm{LiLuF}_{4}(\mathrm{LuLF}), \mathrm{BaY}_{2} \mathrm{~F}_{8}(\mathrm{BYF}), \mathrm{KGd}\left(\mathrm{WO}_{4}\right)_{2}(\mathrm{KGW})$, and $\mathrm{KY}\left(\mathrm{WO}_{4}\right)_{2}(\mathrm{KYW})$ ) was published by the MIT Lincoln Laboratory group [29]. Measurements of the thermal diffusivity, specific heat at constant pressure, thermal conductivity, thermal expansion coefficient, optical path length thermo-optic coefficient, and the thermo-optic coefficient $\mathrm{d} n / \mathrm{d} T$ were presented. This article also reported measurements of the thermal diffusivity and thermal conductivity for $\mathrm{Yb}$ doped samples of $\mathrm{Y}_{3} \mathrm{Al}_{5} \mathrm{O}_{12}, \mathrm{YAlO}_{3}$, and $\mathrm{LiYF}_{4}$ as well, and identified the doping density as an important parameter to consider in the design of cryogenic lasers. Further materials properties measurements by this group have appeared in subsequent publications. In [3], additional thermo-optic measurements are provided for ceramic and single-crystal YAG, GGG, $\mathrm{GdVO}_{4}$, and $\mathrm{Y}_{2} \mathrm{O}_{3}$. Spectroscopic data are also presented for $\mathrm{Yb}: Y A G$ and $Y b: Y L F$. A third publication [30] provides thermo-optic data for the sesquioxide ceramic laser materials $\mathrm{Y}_{2} \mathrm{O}_{3}, \mathrm{Lu}_{2} \mathrm{O}_{3}, \mathrm{Sc}_{2} \mathrm{O}_{3}$, as well as for YLF, YSO, GSAG, and $\mathrm{YVO}_{4}$. 
The modern cryogenic laser period began with the publications of the first experimental cryogenic $\mathrm{Yb}$ :YAG laser [1], and a significantly performance-enhanced $\mathrm{Ti}_{\mathrm{A}} \mathrm{Al}_{2} \mathrm{O}_{3}$ laser [11], both in 1991. Subsequent theoretical and experimental work in the years 1995-1998 confirmed that not only can enhanced laser properties be obtained by cryogenic-cooling, but that dramatic reductions in thermo-optic distortion and enhanced mechanical properties can be realized as well. Thermo-optic properties were not emphasized in the historical era, where the focus was mainly on laser physics, kinetics, and spectroscopy. Thus in our opinion, what is new is that that in the modern era, laser physicists, designers, and engineers have for the first time fully appreciated that the entire set of physical crystal properties (optical, laser kinetics, thermal, thermo-optic, and elastic) must be taken into account to effect successful laser outcomes. Appreciation of this reality has and will continue to drive the demonstration of much higher average power lasers, as well as HAPP lasers, in the future. The concurrent work of our group and that at the MIT Lincoln Laboratory has solved the longest standing problem in the development of solid-state lasers: the near-elimination of deleterious thermal effects. This point cannot be emphasized enough. The manipulation of the thermo-optic properties of solid-state laser hosts through the use of cryogenic-cooling has been proven capable of providing both high average power and near-vanishing thermally-induced aberrations. This results in output beams whose divergence and transverse beam size are virtually constant, without the use of any external beam correction. Beam-parameter independent lasers are highly desirable for harmonic generation, as well as for many other scientific, commercial, and military applications.

In addition to the development of other oxide lasers, and in particular the development of $\mathrm{Yb}$ based lasers, cryogenic-cooling has advanced the performance of Ti: $\mathrm{Al}_{2} \mathrm{O}_{3}$ ultrafast laser technology as well. Unlike $\mathrm{Yb}$ materials like $\mathrm{Yb}: \mathrm{YAG}, \mathrm{Yb}: \mathrm{LuAG}, \mathrm{Yb}: \mathrm{Lu}_{2} \mathrm{O}_{3}, \mathrm{Yb}: \mathrm{YLF}$, and others which all have small quantum defects and thus minimal heat loads, for Ti: $\mathrm{Al}_{2} \mathrm{O}_{3}$ lasers, where optical-pumping usually takes place near $532 \mathrm{~nm}$, the quantum defect for lasing near the peak of the gain profile near $790 \mathrm{~nm}$ is about $33 \%$, a value $>3-5$ times that of typical $\mathrm{Yb}$ based materials. The large heat load of $\mathrm{Ti}_{2} \mathrm{Al}_{2} \mathrm{O}_{3}$ however can be largely be mitigated by cooling the sapphire-based material to $77 \mathrm{~K}$ where the thermal conductivity is dramatically larger, and by the substantial reductions in the thermal expansion and thermo-optic coefficients [11]. The first use of a Ti: $\mathrm{Al}_{2} \mathrm{O}_{3}$ cryogenically-cooled amplifier was reported in [31]; the second-stage amplifier, pumped with $25 \mathrm{~W}$ of $527 \mathrm{~nm}$ power from doubled Nd:YLF lasers, was cooled to $125 \mathrm{~K}$, resulting in an increase of the thermal focal length from 0.45 to $5.6 \mathrm{~m}$, by a factor of about 125. This approach has been discussed and used by a number of researchers [32-35]. The use of cryogenic-cooling in ultrafast lasers will be further discussed later in this article.

\section{Review of Cryogenic-Cooling Benefits}

In this section we review the many benefits that accrue from the implementation of cryogenic-cooling in solid-state lasers. We begin by first examining in Section A. the origin of thermal aberrations in solid-state lasers, in $B$. the most important crystal thermal parameters: the thermal conductivity $k$, followed by the thermal expansion coefficient $\alpha$, and the thermo-optic coefficient $\beta=\mathrm{d} n / \mathrm{d} T$. In $C$. we discuss crystal elastic parameters and in $D$. crystal spectroscopic, kinetic, optical, lasing, nonlinear, and dispersion parameters. We also discuss the motivations for the critical examination of the functional dependence of each parameter on absolute temperature.

\subsection{Thermal Aberrations in Solid-State Lasers}

Crystalline solid-state lasers are optically-pumped devices. The first solid-state laser, demonstrated by Maiman [36], was a flashlamp-pumped ruby laser. Xe and $\mathrm{Kr}$ flashlamps and other similar sources, still in use today for some applications, were the pump source of choice for many decades of the twentieth century, and beginning in the 1980's their dominance has been slowly eroded as narrow band diode pump sources of increasing power have become available. Diode laser technology has developed rapidly and today is the pumping source of choice for most applications, with only the specific cost (Monetary Unit/Watt) preventing its adoption in the highest 
power applications. Typical Xe flashlamps used in inertial confinement fusion (ICF) applications produced output irradiances with black-body temperatures near $9000 \mathrm{~K}$ or more, and with spectral outputs spanning the range of 150 to $1700 \mathrm{~nm}$. Due to the finite number of $\mathrm{Nd}$ absorption bands and the continuous nature of the black-body radiation, the absorption efficiency was poor, with most of the Xe pump radiation being deposited in the pump chamber housing the lasing element, even for typical Nd:Glass compositions where the absorption bands were significantly wider than say Nd:YAG. For the absorbed light, ratios of inversion to heat density were typically only 1.5 , hence for every joule of inversion energy produced, 0.67 joules of heat were generated as well. $\mathrm{Nd}$ lasers dominated the development landscape, and $\mathrm{Yb}$ based lasers were largely ignored due to their poor absorption efficiency. The low repetition rate of ICF laser systems may largely be attributed to the use of flashlamp pump sources. As of this writing, the conversion from flashlamp-pumping to diode-pumping is well under way and accelerating, and modern diode-pumped lasers, many based upon $\mathrm{Yb}$ crystalline materials, produce only about $80 \mathrm{~mJ}$ of heat energy per joule absorbed, a decrease when compared to Nd:Glass laser systems of a factor of over 8 . The reduction in the amount of heat generated is greater than two orders of magnitude when the poor absorption of Xe flashlamp radiation is taken into account.

Thermal aberrations are produced in laser crystals by the production of heat, primarily from the optical-pumping process, by stimulated-emission, and by fluorescence. In some crystals, upconversion, two-photon absorption, non-unity quantum efficiencies, and other effects may also provide additional heating. The total index change $\Delta n_{T}$ in an isotropic (anaxial) laser crystal due to temperature changes may be written as the sum of three terms:

$$
\Delta n_{T}=\Delta n_{\beta}+\Delta n_{\alpha}+\Delta n_{S}
$$

where $\Delta n_{\beta}$ is due to the thermo-optic coefficient $\beta, \Delta n_{\alpha}$ due to the thermal expansion coefficient, and $\Delta n_{S}$ is produced by the stresses (or equivalently the strains) in the crystal. For a laser rod whose barrel is maintained at a constant temperature, the proportional optical phases $\Delta \phi_{r, \varphi}$ in the radial $(r)$ and azimuthal $(\varphi)$ directions due to thermal heating producing a temperature change $\Delta T$ can be written [21]

$$
\begin{aligned}
\Delta \phi_{r, \phi} & =\frac{2 \pi}{\lambda} \cdot \Delta n_{r, \phi} L \\
& =\left(\frac{2 \pi}{\lambda}\right) \cdot\left(\frac{Q_{0}}{4 k}\right)\left[\left[\beta+4 n_{0}^{3} \alpha \cdot C_{0}\right] \cdot r_{0}^{2}-\left[\beta+2 n_{0}^{3} \alpha C_{r, \phi}\right] \cdot r^{2}\right] \cdot L
\end{aligned}
$$

In Equation (2), $L$ is the crystal length, $n_{0}$ the index of refraction, $Q_{0}$ the uniform heat density, $k$ the thermal conductivity, $r_{0}$ the rod radius, and $C_{0}, C_{r}$, and $C_{\varphi}$ are photoelastic constants. It can be seen that the phase varies quadratically with the radius $r$. It is also clear that if $\alpha=\beta=0$, the phases $\Delta \varphi_{r, \varphi}$ would be zero for all $r$, regardless of the value of $Q_{0}$. Equation (2) results from the plane-strain approximation in which the aspect ratio of the $\operatorname{rod}\left(L / 2 r_{0}\right)>>1$. In that approximation, physical changes in the optical pathlength are ignored. In real amplifiers, bulging of the rod faces occurs, an effect that is directly attributable to the thermal expansion coefficient. A similar equation can be derived for the plane stress case $\left(\left(L / 2 r_{0}\right)<<1\right)$, typical of active-mirror or thin disk amplifiers:

$$
\begin{aligned}
\Delta \phi_{r, \varphi} & =\frac{2 \pi}{\lambda} \cdot \Delta n_{r, \phi} L \\
& =\left(\frac{2 \pi}{\lambda}\right) \\
& \cdot\left(\frac{Q_{0}}{4 k}\right)\left[\left[\beta+4 n_{0}^{3} \alpha \cdot C_{0}\right] \cdot r_{0}^{2}+\left[\left(n_{0}-1\right) \alpha(v+1)-2 n_{0}^{3} \alpha C_{r, \phi}\right] \cdot r^{2}\right] \cdot L
\end{aligned}
$$

For the plane-stress case of Equation (3), by inspection again we see that if $\alpha=\beta=0$, the phases $\Delta \phi_{r, \varphi}$ are again zero.In rare cases, most notably for certain Nd:Glass laser compositions with negative 
values of $\beta$, and when stress-induced index changes can be ignored, one can achieve $\Delta n_{T}=0$. We can write Equation (1), ignoring the stress term, as

$$
\Delta n_{T}=\Delta n_{\beta}+\Delta n_{\alpha}=\left[\beta+\left(n_{0}-1\right) \alpha\right] \Delta T
$$

Thus the index variation and resulting phase can be identically zero if

$$
S=\left(\beta+\left(n_{0}-1\right) \alpha\right)=0
$$

This is the condition for athermal operation, and was first employed in connection with the development of $\mathrm{Nd}$ laser phosphate glasses for high-peak-power inertial confinement fusion laser systems to reduce thermal distortion [37].

For cryogenic-cooling, we employ a different strategy. While our goal is to achieve $\Delta \varphi=0$, for most crystals finite values of $\alpha, \beta, v$, and $\mathrm{E}$ at $77 \mathrm{~K}$ allow us to simply approach that condition. As we will see in the following paragraphs, the most successful strategy to date is to reduce the thermal aberrations to residual levels by exploiting the favorable scaling of critical parameters by reducing the operating temperature to the cryogenic regime, which we define here as $<150 \mathrm{~K}$. There are a few crystalline cases in which condition Equation (5) can be approached or satisfied over some temperature range. For Nd:YLF lasers for example, it is well known that one can exploit the negative value of $\beta$ using the $a$-axis $(\sigma)$ transition at $1047 \mathrm{~nm}$. More recent measurements [29] of $\beta$ for the $c$ and $a$ axes of the uniaxial crystal YLF show that negative values of $\beta$ for both axes persist even down to $100 \mathrm{~K}$, allowing the condition Equation (5) to be approached across the entire temperature range for both axes, with $S_{\pi}=-1.88 \times 10^{-6} / \mathrm{K}$ and $S_{\sigma}=1.81 \times 10^{-6} / \mathrm{K}$ at $300 \mathrm{~K}$. At $100 \mathrm{~K}$, one finds that $S_{\pi}=-0.31 \times 10^{-6} / \mathrm{K}$ and $S_{\sigma}=0.56 \times 10^{-6} / \mathrm{K}$, so condition Equation (5) is even more closely approached at $100 \mathrm{~K}$. Another rare example is the closely related material LuLF, a uniaxial crystal which also possesses the property that $\beta$ is negative for the $c$ and a axes [29], however as temperature is lowered both axes become increasingly negative down to about $170 \mathrm{~K}$ where $\beta$ begins to increase, the opposite of YLF in which $\beta$ increases as temperature is lowered. Calculations show that at a temperature near $250 \mathrm{~K}$, Equation (5) can be satisfied identically. As we will see in the data we examine later in this article, for most crystals parameters $k, \beta$, and $\alpha$ scale favorably as temperature is lowered such that thermal aberrations nearly vanish.

For the case where the prime motivation is to eliminate or reduce aberrations to residual levels, we can define two figures-of-merit $\Gamma$ as follows:

$$
\begin{gathered}
\Gamma_{T}=\frac{k}{\alpha \beta \eta_{h}^{T}} \\
\Gamma_{S}=\frac{k(1-v)}{\alpha E \eta_{h}^{T}}
\end{gathered}
$$

Figure of merit $\Gamma_{T}$ addresses changes in the index of refraction due to temperature gradients, and $\Gamma_{S}$ the change in the index due to stress (or strain) effects. To reduce thermal aberrations, we wish to maximize the value of $\Gamma_{T}$. This is achieved by maximizing the value of $k$ to minimize the thermal gradients, minimizing the values of $\alpha$ and $\beta$, and by minimizing the amount of heat generated by minimizing the value of the total heat fraction $\eta_{h}{ }^{T}$. The heat fraction is formally defined as the fraction of the absorbed light converted to heat. It includes contributions from the quantum defect, fluorescence, and stimulated-emission. $\mathrm{Yb}$ based materials are particularly attractive for producing low $\eta_{h}{ }^{T}$ values, due to the lack of concentration-quenching, excited-state absorption, and upconversion, processes that plague other laser materials like Nd:YAG or Nd:YVO ${ }_{4}$, and $\mathrm{Nd}$ :YLF for example. For $\mathrm{Yb}$ based laser materials such as Yb:YAG, we have published a detailed kinetics model [38] that allows the simple calculation of $\eta_{h}{ }^{T}$. The model recognizes two distinct heating mechanisms, the first due to 
Boltzmann heating (or cooling), and the second due to the photon related processes of fluorescence and stimulated-emission. In general, the heat fraction for Yb:YAG is defined by the equation

$$
\eta_{h}^{T}=\frac{Q_{h}^{B}+Q_{h}^{P}}{Q_{A}}=A+B \eta_{e x}+C\left(1-\eta_{e x}\right)
$$

where $Q_{A}$ is the total absorbed power density, $Q_{h}^{B}$ the Boltzmann heat density, and $Q_{h}^{P}$ the photon heat density. The quantities $A, B$, and $C$ are constants and $\eta_{e x}$ is the laser extraction efficiency. The constants $A, B$, and $C$ are associated with Boltzmann heating or cooling, stimulated-emission, and fluorescence respectively. For complete laser extraction only the first and second terms contribute, since the fluorescence is zero, whereas for no laser extraction the first and third terms contribute. In most cases the extraction efficiency is between those two limits, and Equation (8) allows the simple calculation of the total heat load when both stimulated-emission and fluorescence are operative.

Equation (7) is the figure of merit for considering stress effects only. It arises naturally from the consideration of the stresses generated in laser crystals due to thermal gradients. For a rod amplifier, using the plane-strain approximation [21], the stresses can be calculated from the well-known equations

$$
\begin{aligned}
\sigma_{r r} & =\frac{Q_{0}}{16 M_{s}}\left(r^{2}-r_{0}^{2}\right) \\
\sigma_{\varphi \varphi} & =\frac{Q_{0}}{16 M_{s}}\left(3 r^{2}-r_{0}^{2}\right) \\
\sigma_{z z} & =\frac{Q_{0}}{8 M_{s}}\left(2 r^{2}-r_{0}^{2}\right)
\end{aligned}
$$

and for a slab amplifier in the plane-strain approximation, from [22]

$$
\sigma_{x x}=\sigma_{z z}=\frac{Q_{0}}{2 M_{s}}\left(2 y^{2}-\frac{t^{2}}{12}\right)
$$

Equations (9)-(12) show that the obtained stress is proportional to the heat density and inversely proportional to $M_{s}$, a quantity known as the materials parameter, and expressed as

$$
M_{S}=\frac{k(1-v)}{\alpha E}
$$

The materials parameter is then a mixture of thermal and elastic constants. To reduce the stress one wants to maximize the value of $M_{s}$, and that is the motivation for the use of the figure of merit $\Gamma_{S}$. To minimize stress contributions to the change in the index of refraction, the appropriate strategy is to maximize $k$, minimize $\alpha, E$, and $\eta_{h}^{T}$, and minimize the value of $v$. While other similar figures of merit have been used [3], we prefer our approach where the contributing factors to the change in index of refraction from purely thermal effects and thermally-induced stress are clearly separated. If desired, however, after eliminating duplicate parameters ( $k$ and $\alpha$ in Equations (6) and (7)) we can write a simple overall multiplicative figure-of-merit $\Gamma$ as

$$
\Gamma=\Gamma_{T} \cdot \Gamma_{S} \rightarrow \frac{k(1-v)}{\alpha E \beta \eta_{h}}=\frac{M_{S}}{\beta \cdot \eta_{h}}
$$

Laser materials with the highest figure of merit therefore should have a large value for $M_{S}$, and minimum values for $\beta$ and $\eta_{h}$. 


\subsection{Thermal Parameters}

\subsubsection{Thermal Conductivity}

Thermal conductivity $k$ is a very important laser design parameter, and understanding its' dependence on temperature and dopant density is crucial to implementing cryogenic laser designs. We first examine the standard quadratic equation used for determining the $\mathrm{CW}$ temperature distribution in a rod or slab laser [21,22]. For a slab laser the temperature $T(y)$ as a function of slab thickness is given by

$$
T(y)=T_{0}-\frac{Q_{0} y^{2}}{2 k}
$$

The slab center temperature is $T_{0}, Q_{0}$ is the (assumed uniform) heat power density, $k$ the thermal conductivity, and $y$ the distance from the slab center, where $0 \leqslant y \leqslant(t / 2)$ and $t$ is the slab thickness. Newton's law of cooling is used as he boundary condition at $y= \pm t / 2$.To reduce the temperature differential between the center and the edge of the slab, important in reducing the thermal aberrations, we want to reduce or eliminate the thermal gradient given by

$$
\frac{d T}{d y}=-\frac{Q_{0}}{k} y
$$

From Equation (16) we see that reducing the thermal gradient is equivalent to reducing the ratio $\left(Q_{0} / k\right)$. Reducing the heat fraction in a laser material reduces $Q_{0}$; the other way to reduce the gradient is to increase $k$. Both of these strategies are used in modern diode-pumped lasers; for diode-pumped $\mathrm{Yb}$ lasers in particular, heat fractions are much reduced when compared to older materials such as $\mathrm{Nd}: Y A G, \mathrm{Nd}: Y L F$, or Nd:YVO 4 . Here we recount how cryogenic-cooling has been used to obtain much larger $\mathrm{k}$ values. We can also see from Equation (16) that for flashlamp-pumped lasers where $Q_{0}$ values can be an order of magnitude or two larger than the modest values obtained with diode-pumped lasers, thermal gradients are very much larger.

Thermal aberrations can be substantially reduced by using diode-pumping to reduce the heat fraction, and cryogenic-cooling to increase $k$. In Equation (15), the thermal conductivity is treated as a constant, independent of temperature or doping density. One can include the temperature dependence using a number of methods. We may write the CW heat equation for an infinite slab element as

$$
-\frac{d}{d y}\left[k(T) \cdot \frac{d T}{d y}\right]=Q_{0}
$$

As we will explore later in this article, theoretically the thermal conductivity varies approximately as $(1 / T)$ in the region of interest from room temperature to $77 \mathrm{~K}$. We can write a simple relationship for the thermal conductivity dependence on $T$ as

$$
k(T)=k_{0} \cdot \frac{T_{A}}{T}
$$

where $k_{0}$ is the thermal conductivity at ambient temperature $T_{A}=300 \mathrm{~K}$. Substituting in Equation (16) and integrating results in the analytical solution

$$
T(y)=T_{0} \cdot \operatorname{Exp}\left(-\frac{Q_{0} y^{2}}{2 k_{0} T_{A}}\right)
$$

This equation allows the computation of the temperature in any slab in which the thermal conductivity varies as $(1 / T)$. It should be noted that, as first reported in [20], the equation is then 
no longer quadratic. The derived equation satisfies the boundary condition that $T(y=0)=T_{0}$. For $y= \pm t / 2$, we find the edge temperature $T_{e}$ :

$$
T_{e}=T_{0} \cdot \operatorname{Exp}\left(-\frac{Q_{0} t^{2}}{8 k_{0} T_{A}}\right)
$$

Equation (20) allows the calculation of the edge temperature, knowing $T_{0}$. Often $T_{e}$ is fixed at room temperature by a temperature controlled water chiller, or by liquid nitrogen $\left(\mathrm{LN}_{2}\right)$, and in that case Equation (20) may be used to calculate the slab center temperature $T_{0}$. It should be noted that as expected, for small $Q_{0}$, Equation (20) can be expanded in a Taylor series. Keeping only the first term, we find

$$
T(y)=T_{0}\left(1-\frac{Q_{0} y^{2}}{2 k_{0} T_{A}}\right)=\lim _{\left(T_{0} / T_{A}\right) \rightarrow 1} T_{0}-\frac{Q_{0} y^{2}}{2 k_{0}} \cdot\left[\frac{T_{0}}{T_{\mathrm{A}}}\right]=T_{0}-\frac{Q_{0} y^{2}}{2 k_{0}}
$$

or the equation reverts to the $k=$ constant case. For any finite value of $Q_{0}$, the temperature, and thus the thermal aberrations are not quadratic with $y$, and thus not correctable using a simple lens. From a practical standpoint, for lower values of $Q_{0}$, this effect can be ignored.

Equation (19) may be differentiated, to find the temperature gradient

$$
\frac{d T}{d y}=-\left[\frac{T_{0}}{T_{A}}\right] \cdot\left[\frac{Q_{0}}{k_{0}}\right] y \cdot \operatorname{Exp}\left(-\frac{Q_{0} y^{2}}{2 k_{0} T_{A}}\right)
$$

Here we see that reducing the ratio $\left(Q_{0} / k_{0}\right)$ is required in this case as well to reduce the thermal profile, except that in this more realistic case, the decrease in the thermal gradient is faster that in the standard case. Figure 1 shows calculation results from the standard Equations (15) and (19) derived here. Temperature as a function of the slab thickness coordinate $y$ is shown for three cases of heat density, 500, 1500, and $2500 \mathrm{~W} / \mathrm{cm}^{3}$, and for a slab edge temperature of $300 \mathrm{~K}$. Note that we assume that Equation (18) is valid above room temperature as well. The same plot is shown in Figure 2 for an edge temperature of $80 \mathrm{~K}$. The thermal conductivity is scaled according to Equation (18), and was assumed to be $0.112 \mathrm{~W} /(\mathrm{cm} \cdot \mathrm{K})$ at $300 \mathrm{~K}$, and $0.424 \mathrm{~W} /(\mathrm{cm} \cdot \mathrm{K})$ at $80 \mathrm{~K}$. The difference between the standard model and ours is small at $500 \mathrm{~W} / \mathrm{cm}^{3}$ and below, whereas at the largest heat densities of 1500 and $2500 \mathrm{~W} / \mathrm{cm}^{3}$, the difference strongly increases. For $300 \mathrm{~K}$, the difference between the two models for $2500 \mathrm{~W} / \mathrm{cm}^{3}$ amounts to $23.2 \mathrm{~K}$; for $80 \mathrm{~K}$ the difference is 6.10 degrees. The fundamental reason for the temperature increase using our model is the decrease in the thermal conductivity that occurs as one moves towards the slab center where the temperature is maximum. This effect is ignored in the standard model. As the ambient or cooling temperature decreases to $80 \mathrm{~K}$, the enhanced thermal conductivity for all temperatures reduces the difference between the two models.

We have solved the CW heat equation, ignoring the $z$-dependence, for rod elements as well, and find the following similar equation:

$$
T(r)=T_{0} \cdot \operatorname{Exp}\left(-\frac{Q_{0} r^{2}}{4 T_{A}}\right)
$$

For cases in which the thermal conductivity does not vary as $(1 / T)$, but with a power of $T\left(1 / T^{b}\right)$ for example, we can still obtain an analytical solution, albeit a transcendental one. The solution for a slab with $b>1$ is

$$
T(y)=\frac{T_{0}}{\left[\frac{Q_{0}(b-1) y^{2}}{2 k} \cdot \frac{T_{0}^{1-b}}{T_{A}}\right]^{\frac{1}{1-b}}}
$$


whereas before $T_{0}$ is the temperature for $y=0$, and $T_{A}$ is the ambient temperature. For $y=t / 2$, we can find the edge temperaure $T_{e}$, as

$$
T_{e}=\frac{T_{0}}{\left[\frac{Q_{0}(b-1) t^{2}}{8 k} \cdot \frac{T_{0}^{1-b}}{T_{A}}\right]^{\frac{1}{1-b}}}
$$

This equation is transcendental in $T_{0}$, and may be solved numerically for any edge temperature for $T_{0}$ after $Q_{0}, b, k$, and $T_{A}$ are specified.

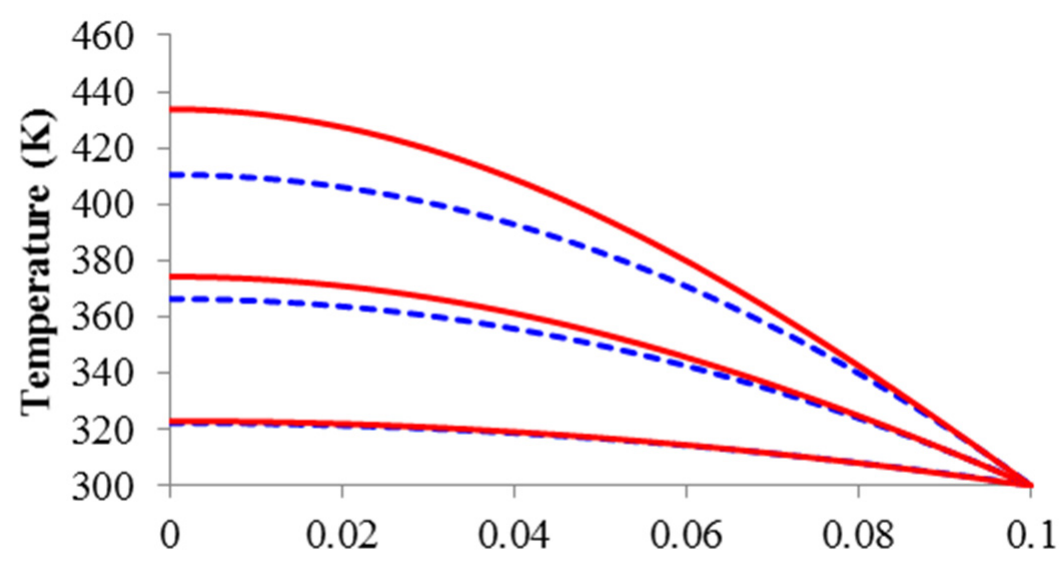

Thickness Coordinate y $(\mathrm{cm})$

Figure 1. Temperature as a function of thickness coordinate $y$, for the standard model (blue) and the new model described here, for heat density values of 500,1500, and $2500 \mathrm{~W} / \mathrm{cm}^{3}$ (bottom to top) and an ambient cooling temperature of $300 \mathrm{~K}$.

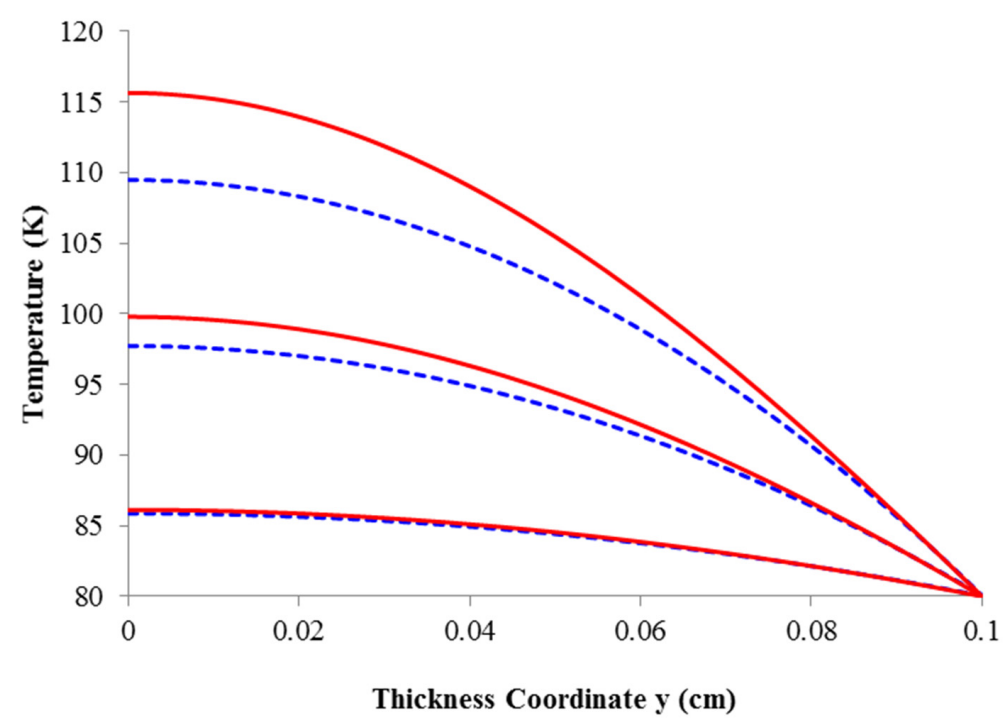

Figure 2. Temperature as a function of thickness coordinate $y$, for the standard model (blue) and the new model described here, for heat density values of 500, 1500, and $2500 \mathrm{~W} / \mathrm{cm}^{3}$ (bottom to top) and an ambient cooling temperature of $80 \mathrm{~K}$. 


\subsubsection{Thermal Conductivity Standard Debye Model}

Using the kinetics theory of gases, applied to a phonon gas, and the Debye specific heat theory [39], the thermal conductivity $k$ in its simplest form can be written as

$$
k=\frac{1}{3} C v_{s} \Lambda=\frac{1}{3} C v_{s}^{2} \tau
$$

where $C$ is the specific heat at constant volume, per unit volume. $C$ is obtained from the relationship

$$
C=C_{V} \rho
$$

Here $C_{V}$ is the specific heat per unit volume per unit mass and $\rho$ the mass density. Note that for dielectric crystals, the specific heats for constant volume and pressure are normally assumed to be equal $\left(C_{V}=C_{P}\right) \cdot v_{S}$ is the mean phonon speed in the crystal, and $\Lambda$ is the phonon mean-free path. $v_{S}$ can be calculated for any crystal using the sound speeds for the acoustic transverse and longitudinal waves, $v_{t}$ and $v_{l}$ respectively, and accounting for the fact that there are twice as many transverse as longitudinal waves, from

$$
v_{s}=\left[\frac{1}{3}\left(\frac{2}{v_{t}^{3}}+\frac{1}{v_{l}^{3}}\right)\right]^{-1 / 3}
$$

The transverse and longitudinal sound speeds may be calculate using the elastic coefficients, or equivalently for anaxial crystals, the bulk and shear moduli and the mass density. For $Y A G\left(\mathrm{Y}_{3} \mathrm{Al}_{5} \mathrm{O}_{12}\right)$ and $\mathrm{YbAG}\left(\mathrm{Yb}_{3} \mathrm{Al}_{5} \mathrm{O}_{12}\right)$, which we will discuss later in this paper, a recent publication [40] provides values for $v_{t}$ and $v_{l}$, as well as for the bulk and shear moduli, Poisson's ratio, Pugh's ratio, and Young's modulus.

$\tau$ is the phonon relaxation time, which for undoped crystals is the Umklapp relaxation time. In the Debye approximation, the total phonon energy can be expressed as

$$
S(\omega)=\left[\frac{9 n N_{A} \rho}{M}\right] \cdot\left[\frac{\hbar}{\omega_{D}^{3}}\right] \cdot \int_{0}^{\omega_{D}} \frac{\omega^{3}}{\left[e^{\frac{\hbar \omega}{k_{B} T}}-1\right]} d \omega
$$

$n$ is the number of atoms in the crystal chemical formula, $N_{A}$ Avogadro's number, $M$ the molecular mass /mole, $\hbar$ Planck's constant divided by $2 \pi, \omega_{D}$ the Debye frequency, $k_{B}$ the Boltzmann constant, $\omega$ the angular phonon frequency, $T$ is the absolute temperature and $\rho$ the mass density. The derivative of Equation (29) with respect to temperature yields the specific heat per unit volume:

$$
C=\frac{d S(\omega)}{d T}=\left[\frac{9 n N_{A} \rho}{M}\right] \cdot\left[\frac{\hbar^{2}}{k_{B}^{2} \omega_{D}^{3} T^{2}}\right] \cdot \int_{0}^{\omega_{D}} \frac{\omega^{4} e^{\frac{\hbar \omega}{k_{B} T}}}{\left[\frac{\hbar \omega}{e^{\frac{k_{B} T}{2}}-1}\right]^{2}} d \omega
$$

The Debye frequency is related to the Debye temperature $\theta_{D}$ through the relationship

$$
\theta_{D}=\frac{\hbar \omega_{D}}{k_{B}}
$$


If we define the ratios $x=\hbar \omega / k_{B} T$ and $x_{D}=\hbar \omega_{D} / k_{B} T$, divide by $\rho$, and paramaterize Equation (29), we find the standard Debye expression for the specific heat [39]:

$$
C_{V}=\left[\frac{9 n N_{A}}{M}\right] \cdot\left(\frac{T}{\theta_{D}}\right)^{3} \cdot \int_{0}^{x_{D}} \frac{x^{4} e^{x}}{\left[e^{x}-1\right]^{2}} d x
$$

where $x$ is integrated from 0 to $x_{D}$.

There are two often-used limits to Equation (32). For large temperature values $\left(T>>\theta_{D}\right)$, the exponential function may be expanded in a Taylor series, with the result being the constant

$$
C_{V}=\frac{3 n N_{A} k_{B}}{M}
$$

This result is known as the Dulong-Petit law. As an approximation to the Debye model, the following equation is often used for any $T$ [41]:

$$
C_{V}=\left[\frac{9 n N_{A} k_{B}}{M}\right] \cdot \frac{1}{1+\frac{5}{4 \pi^{4}}\left(\frac{\theta_{D}}{T}\right)^{3}}
$$

If we take the limit as $T \rightarrow 0$, we find the following equation

$$
C_{V} \simeq\left(\frac{12 \pi^{4}}{5}\right) \cdot\left(\frac{n N_{A} k_{B}}{M}\right)\left[\frac{T}{\theta_{D}}\right]^{3}
$$

This equation correctly predicts the $T^{3}$ dependence of the specific heat for low temperatures, and is known as the Debye- $T^{3}$ law.

In Figure 3, we show a plot of $C_{V}$ for the undoped laser material $\mathrm{Y}_{3} \mathrm{Al}_{5} \mathrm{O}_{12}$, as a function of temperature. We used Equation (32) for calculating $C_{V}$ using the Debye model, and the YAG Debye temperature of $760 \mathrm{~K}$ [29]. Also shown are published data for YAG [29], as well as the approximate results obtained using Equation (34). The Debye model matches the experimental data fairly well, while the approximate model over estimates the specific heat for temperatures starting at about $180 \mathrm{~K}$.

Rather than Equation (26), an alternative approach to calculating the thermal conductivity was used by Klemens [42], whose model we will discuss in more detail in the next section of this paper. The following more sophisticated relationship for $\mathrm{k}$ can be employed:

$$
k=\frac{1}{3} \int S(\omega) v_{s}^{2} \tau(\omega) d \omega
$$

where $S(\omega)$ is defined by Equation (29), and $v_{s}$ and $\tau$ have been previously defined. The use of $\tau(\omega)$ rather than the mean free path makes it simple to introduce phonon scattering from impurity atoms introduced into the crystal lattice, as we will review in the following section. If we take $S(\omega)$ in the high temperature limit, we find the following relationship:

$$
S(\omega)=\frac{3}{2} \frac{k_{B} \omega^{2}}{\pi^{2} v_{S}^{3}}
$$

where we have used the following expression for the Debye frequency:

$$
\omega_{D}=2 \pi\left[\frac{3 n N_{A} \rho}{M}\right]^{1 / 3} \cdot v_{S}
$$


Inserting Equation (37) into Equation (36), and assuming that the phonon speed $v_{s}$ is a constant, we obtain

$$
k=\frac{k_{B}}{2 \pi^{2} v_{s}} \cdot \int_{0}^{\omega_{D}} \omega^{2} \tau(\omega) d \omega
$$

This equation has been used by Klemens [42] to calculate $\mathrm{k}$ when scattering defects are doped into a crystal. It should be noted however that this equation is strictly valid only when $T>\theta_{D}$. For most laser materials, and particularly in the temperature range of interest here, about $77-300 \mathrm{~K}$, this condition is violated but nevertheless often used because it can provide analytical results.

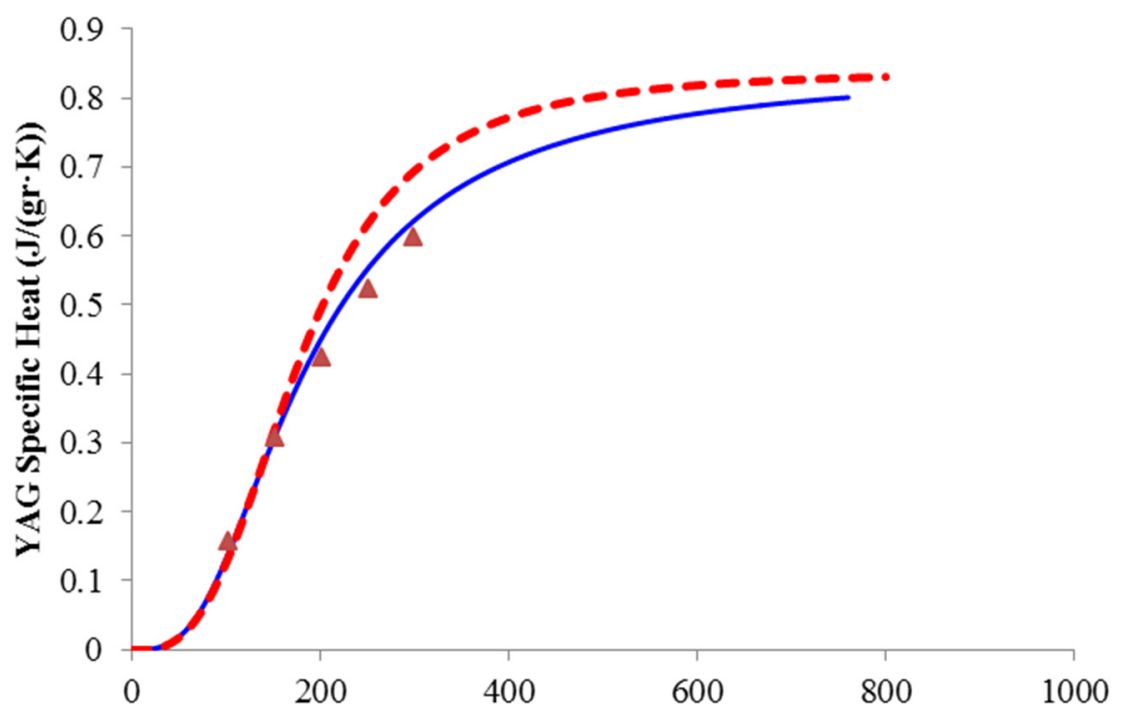

\begin{abstract}
Absolute Temperature (K)
Figure 3. Specific heat for the undoped laser material $\mathrm{Y}_{3} \mathrm{Al}_{5} \mathrm{O}_{12}$ as a function of temperature. Shown are the calculated values (solid blue line) using Equation (32), the values obtained with the specific heat approximation of Equation (34) (dashed red line), and the experimental data from [29].
\end{abstract}

\title{
2.2.3. Thermal Conductivity: The Influence of Dopant Density
}

The thermal conductivity of doped laser materials is strongly affected by the presence of impurities, and in particular the doping density of lasing ions. Small quantities of dopant ions have a minimal effect on thermal conductivity, while ever increasing dopant densities degrade the thermal conductivity in an inverse relationship. This may be seen in Figure 4 wherein we display the thermal conductivity as a function of absolute temperature for the laser material YAG, for the undoped case, and for $\mathrm{Yb}$ concentrations of 2, 4, 10, 15, and 25 at. \%. The 2, 4, and $15 \mathrm{Yb}$ at. \% values are obtained from [29], and the 10 and 25 at. \% values are estimated from computer best fits to the data of [29] for each temperature. If we examine the data at $100 \mathrm{~K}$ for example, compared with the room temperature data at $300 \mathrm{~K}$, we see that for the undoped sample the thermal conductivity value is $0.461 \mathrm{~W} /(\mathrm{cm} \cdot \mathrm{K})$, whereas the thermal conductivity for 10 at. $\% \mathrm{Yb}$ is $0.190 \mathrm{~W} /(\mathrm{cm} \cdot \mathrm{K})$, and for 25 at. \% $\mathrm{Yb}$ only $0.120 \mathrm{~W} /(\mathrm{cm} \cdot \mathrm{K})$. Thus at $100 \mathrm{~K}$ the drop in thermal conductivities amount to reductions of 0.41 and 0.26 respectively, when compared to the undoped value. The thermal conductivity at $300 \mathrm{~K}$ for the undoped case is $0.112 \mathrm{~W} /(\mathrm{cm} \cdot \mathrm{K})$, and $0.061 \mathrm{~W} /(\mathrm{cm} \cdot \mathrm{K})$ for the 25 at. \% Yb doping case. Thus if we compare the undoped cases at $100 \mathrm{~K}$ and $300 \mathrm{~K}$, we find the thermal conductivity larger by a factor of 4.1 at $100 \mathrm{~K}$. For the 25 at. \% Yb case however, if we compare the $100 \mathrm{~K}$ and the $300 \mathrm{~K}$ data, we see that the thermal conductivity rises by a factor of about 1.97 between 300 and $100 \mathrm{~K}$, but also that the thermal conductivity at $300 \mathrm{~K}$ is smaller by a factor of about 0.545 when compared with the undoped case at $300 \mathrm{~K}$. Most Yb:YAG lasers use Yb dopings between 2 and 10 at. \%. For the 10 at. \% Yb case, the 
thermal conductivity at $100 \mathrm{~K}$ is larger by a factor of about 2.57 , a factor of 1.6 times smaller than the increase of 4.1 between 300 and $100 \mathrm{~K}$ for undoped YAG.

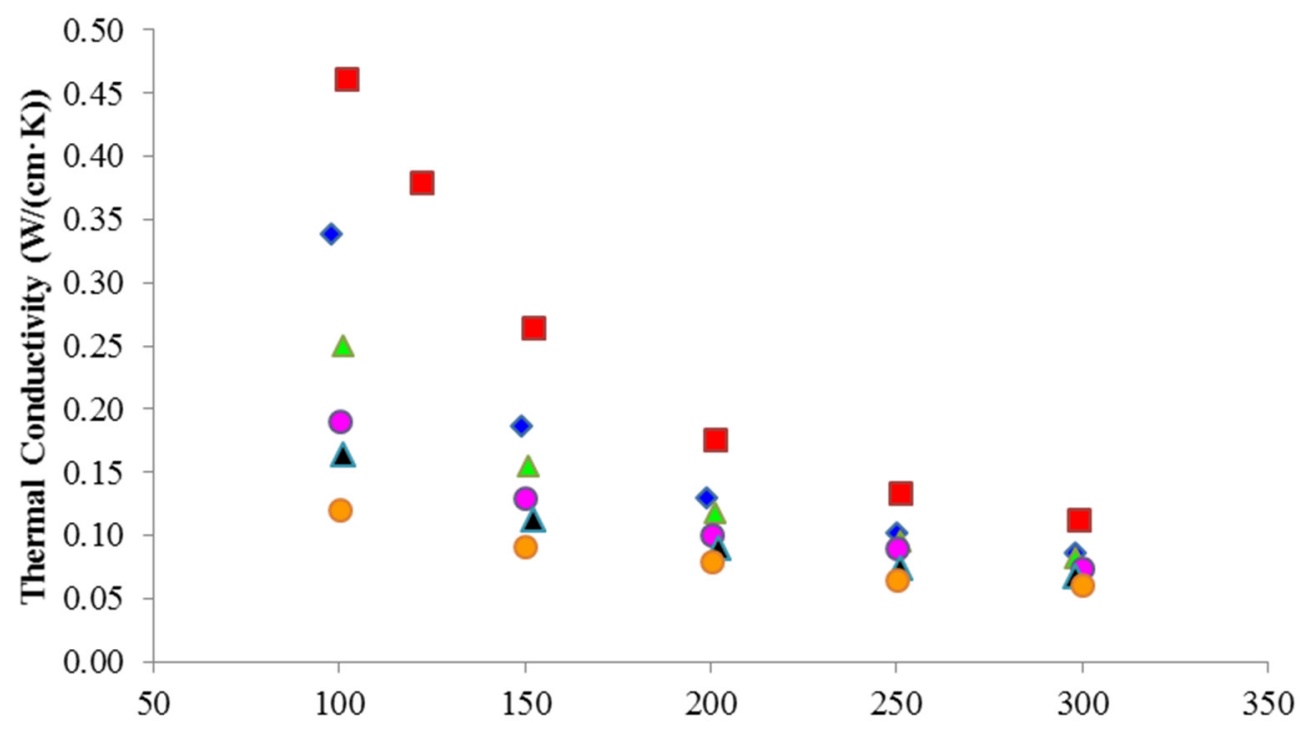

Absolute Temperature (K)

Figure 4. Thermal conductivity as a function of absolute temperature for undoped single-crystal Czochralski-grown YAG (red), and Yb:YAG with doping densities of 2 (blue), 4 (green), 10 (pink), 15 (black), and 25 (orange) at. \%. The experimental data for the 2, 4 and 15 at. \% values are taken from [29], while the 10 and 25 at. \% values were determined from fits to the data of [29] for each temperature.

It has long been known [42] that the mass of a dopant (donor) atom ( $\mathrm{Yb}$ for example), replacing an acceptor atom ( $\mathrm{Y}$ for example) in a crystal lattice results in a decrease in thermal conductivity, as illustrated in Figure 4. Recently, attempts have been made to further quantify the effect of donor-acceptor mismatch. It has been demonstrated experimentally, for example, that if the donor atom mass is well-matched to the acceptor ion, the decrease in thermal conductivity as doping density increases is minimized. A particularly good example of this is the crystal $\mathrm{Yb}_{2} \mathrm{Lu}_{2} \mathrm{O}_{3}$, where the mismatch between $\mathrm{Yb}$ and $\mathrm{Lu}$ is small. The data are shown in Figure 5, where $\mathrm{k}$ is displayed as a function of absolute temperature for undoped $\mathrm{Lu}_{2} \mathrm{O}_{3}$ and for 11 at. \% $\mathrm{Yb}$ doping [43]. At $306 \mathrm{~K}$, the difference between the undoped point and the two adjacent 11 at. \% Yb-doped points amounts to an average of only $9.5 \%$. In the region between 96 and $106 \mathrm{~K}$, the doped and undoped $\mathrm{k}$ values are nearly indistinguishable. In Table 1 , we show the acceptor minus the donor mass mis-match between 11 common dopants and 10 acceptor atoms. For the case of $\mathrm{Yb}: \mathrm{Lu}_{2} \mathrm{O}_{3}$ for example the mass-mismatch between $\mathrm{Lu}$ and $\mathrm{Yb}$ is only 1.93 amu's. The data of Figure 5 reflect the close match between $\mathrm{Lu}$ and $\mathrm{Yb}$ ions, and high thermal conductivity at $80 \mathrm{~K}$ is one of the reasons $\mathrm{Yb}: \mathrm{Lu}_{2} \mathrm{O}_{3}$ has become a very desirable high-power laser material. Table 1 also shows that $\mathrm{Lu}$ is a very good match for Er and $\mathrm{Tm}$, and that $\mathrm{Ce}, \mathrm{Pr}$, and $\mathrm{Nd}$ are very good matches to $\mathrm{La}$. In addition to $\mathrm{Lu}_{2} \mathrm{O}_{3}$, two other sesquioxides, $\mathrm{Sc}_{2} \mathrm{O}_{3}$ and $\mathrm{Y}_{2} \mathrm{O}_{3}$, have been intensely investigated as newer laser materials. No donor results in a good match for $\mathrm{Y}$. For Sc, the rare-earths are a very poor mass match, and this is also reflected in the experimental data. $\mathrm{Yb}$ has the worst mis-match, and $\mathrm{Er}, \mathrm{Tm}$, and Ho are also poor. The poor mismatches of these donor-acceptor ions are reflected in the experimental data as well, as shown in Figure 6 where thermal conductivity is shown as a function of absolute temperature [43,44]. Data are plotted for undoped hydrothermally-grown and Czochralski-grown undoped $\mathrm{Sc}_{2} \mathrm{O}_{3}$, for 2.9 and 9 at. \% Yb doping and for 3 at. \% Er doping. As expected from Table 1, there is a strong drop in $\mathrm{k}$ for Er and an even worse drop for $\mathrm{Yb}$, for all temperatures. Another interesting comparison is Yb:YAG with Yb:LuAG. The mass mismatch for $\mathrm{Yb}$ and $\mathrm{Y}$ is about $-84.13 \mathrm{amu}$. For $\mathrm{Yb}$ and Lu it is only $1.93 \mathrm{amu}$. Based on this 
comparison we would expect the mass scattering to be much smaller in Yb:LuAG than in Yb:YAG. We will see in Section 2.2.7 that as $\mathrm{Yb}$ doping increases, the falloff in thermal conductivity for $\mathrm{Yb}: \mathrm{LuAG}$ is much smaller than for Yb:YAG.

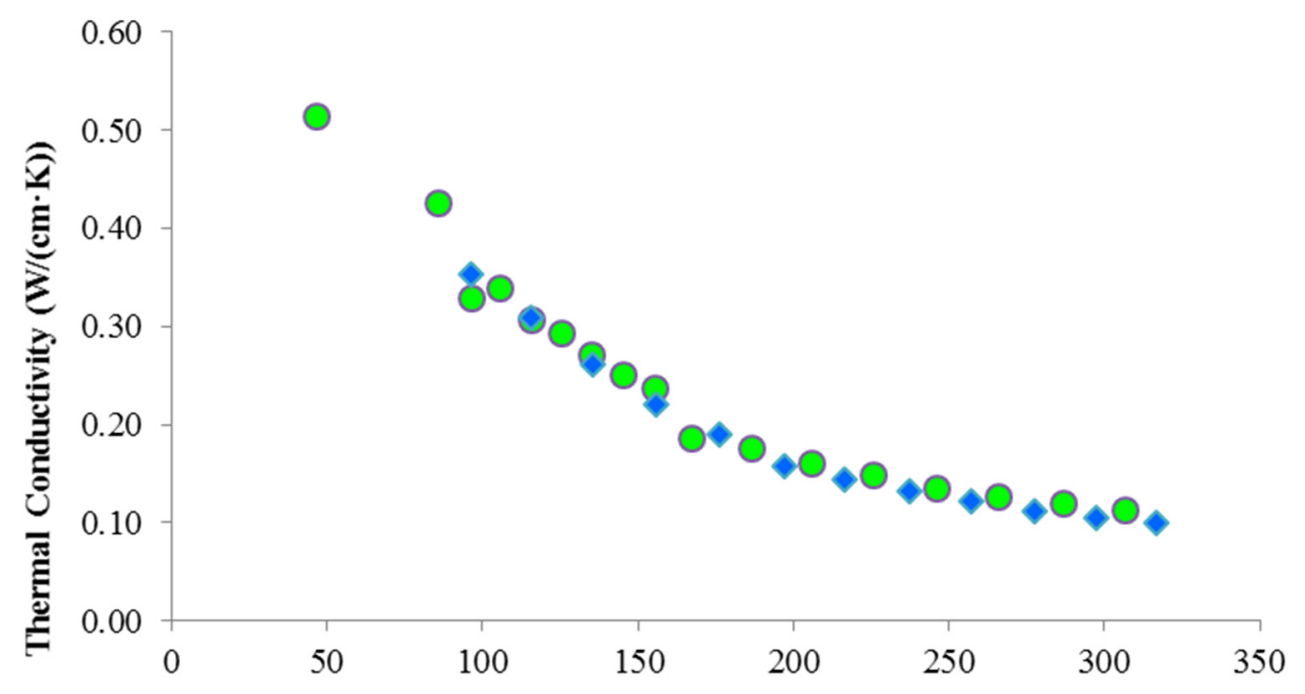

Absolute Temperature (K)

Figure 5. Thermal conductivity as a function of absolute temperature for hydrothermally-grown

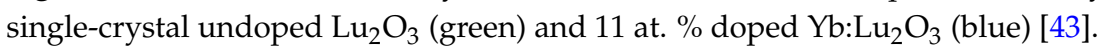

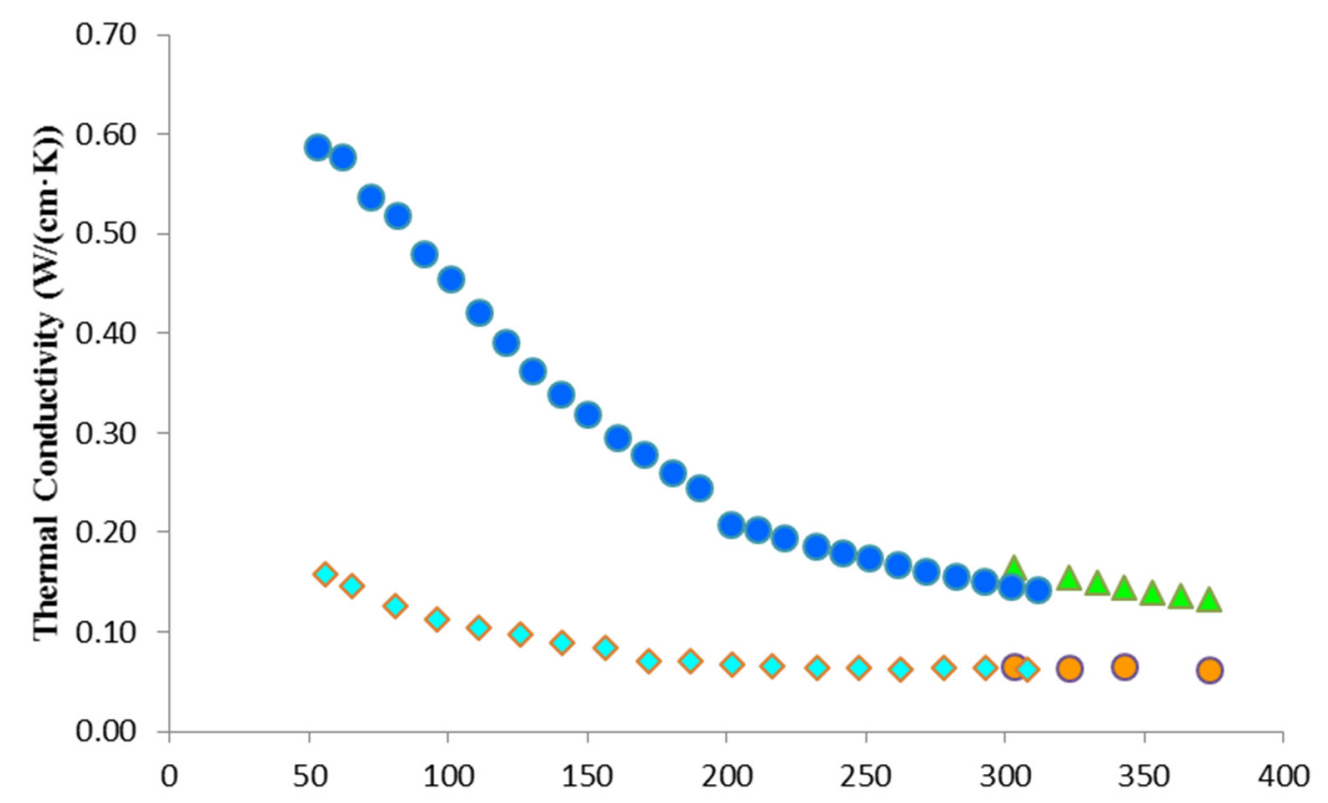

Absolute Temperature (K)

Figure 6. Thermal conductivity as a function of absolute temperature for the sesquioxide material $\mathrm{Sc}_{2} \mathrm{O}_{3}$, for undoped hydrothermal (blue) [43] and Czochralski (green) [44] grown single-crystals, 3 at. \% Er doping (light blue) [44], and 2.8 at. \% Yb doping (orange) [44]. 
Table 1. Donor-Acceptor atomic numbers, weights, and weight differences for common dopants (donors) and acceptors.

\begin{tabular}{ccccccccccccc}
\hline & & Acceptor & $\mathbf{L i}$ & $\mathbf{M g}$ & $\mathbf{A l}$ & $\mathbf{K}$ & $\mathbf{C a}$ & $\mathbf{S c}$ & $\mathbf{Y}$ & $\mathbf{L a}$ & $\mathbf{G d}$ & $\mathbf{L u}$ \\
\cline { 3 - 11 } Donor & At. Number & At. Number & $\mathbf{3}$ & $\mathbf{1 2}$ & $\mathbf{1 3}$ & $\mathbf{1 9}$ & $\mathbf{2 0}$ & $\mathbf{2 1}$ & $\mathbf{3 9}$ & $\mathbf{5 7}$ & $\mathbf{6 4}$ & $\mathbf{7 1}$ \\
\cline { 3 - 10 } & & At. Weight (amu) & $\mathbf{6 . 9 4}$ & $\mathbf{2 4 . 3 1}$ & $\mathbf{2 6 . 9 8}$ & $\mathbf{3 9 . 1 0}$ & $\mathbf{4 0 . 0 8}$ & $\mathbf{4 4 . 9 6}$ & $\mathbf{8 8 . 9 1}$ & $\mathbf{1 3 9 . 9 1}$ & $\mathbf{1 5 7 . 2 5}$ & $\mathbf{1 7 4 . 9 7}$ \\
\hline $\mathrm{Ti}$ & 22 & 47.87 & -44.87 & 56.87 & -20.89 & -8.77 & -7.79 & -2.91 & 41.04 & 92.04 & 109.38 & 127.10 \\
$\mathrm{~V}$ & 23 & 50.94 & -47.94 & 59.94 & -23.96 & -11.84 & -10.86 & -5.99 & 37.96 & 88.96 & 106.31 & 124.03 \\
$\mathrm{Cr}$ & 24 & 52.00 & -49.00 & 61.00 & -25.01 & -12.90 & -11.92 & -7.04 & 36.91 & 87.91 & 105.25 & 122.97 \\
$\mathrm{Co}$ & 27 & 58.93 & -55.93 & 67.93 & -31.95 & -19.84 & -18.86 & -13.98 & 29.97 & 80.97 & 98.32 & 116.03 \\
$\mathrm{Ni}$ & 28 & 58.69 & -55.69 & 67.69 & -31.71 & -19.60 & -18.62 & -13.74 & 30.21 & 81.21 & 98.56 & 116.27 \\
$\mathrm{Ce}$ & 58 & 140.12 & -137.12 & 149.12 & -113.13 & 101.02 & -100.04 & -95.16 & -51.21 & -0.21 & 17.14 & 34.85 \\
$\mathrm{Pr}$ & 59 & 140.91 & -137.91 & 149.91 & -113.93 & 101.81 & -100.83 & -95.95 & -52.00 & -1.00 & 16.34 & 34.06 \\
$\mathrm{Nd}$ & 60 & 144.24 & -141.24 & 153.24 & -117.26 & 105.14 & -104.16 & -99.28 & -55.33 & -4.33 & 13.01 & 30.73 \\
$\mathrm{Sm}$ & 62 & 150.36 & -147.36 & 159.36 & -123.38 & 111.26 & -110.28 & 105.40 & -61.45 & -10.45 & 6.89 & 24.61 \\
$\mathrm{Eu}$ & 63 & 151.97 & -148.97 & 160.97 & -124.98 & 112.87 & -111.89 & 107.01 & -63.06 & -12.06 & 5.28 & 23.00 \\
$\mathrm{Dy}$ & 66 & 162.50 & -159.50 & 171.50 & -135.52 & 123.40 & -122.42 & 117.54 & -73.59 & -22.59 & -5.25 & 12.47 \\
$\mathrm{Ho}$ & 67 & 164.93 & -161.93 & 173.93 & -137.95 & 125.83 & -124.85 & 119.97 & -76.02 & -25.02 & -7.68 & 10.04 \\
$\mathrm{Er}$ & 68 & 167.26 & -164.26 & 176.26 & -140.28 & 128.16 & -127.18 & 122.30 & -78.35 & -27.35 & -10.01 & 7.71 \\
$\mathrm{Tm}$ & 69 & 168.93 & -165.93 & 177.93 & -141.95 & 129.84 & -128.86 & 123.98 & -80.03 & -29.03 & -11.68 & 6.03 \\
$\mathrm{Yb}$ & 70 & 173.04 & -170.04 & 182.04 & -146.06 & 133.94 & -132.96 & 128.08 & -84.13 & -33.13 & -15.79 & 1.93 \\
\hline
\end{tabular}




\subsubsection{Thermal Conductivity Temperature Dependence}

Many thermal conductivity models use the Debye high temperature limit of Equation (33). Since then $C_{v}$ is independent of temperature, the phonon speed is nearly independent of temperature, and the mean free path is inversely proportional to the temperature $T$, we find that in the high temperature region

$$
k_{2}=k_{1} \cdot\left(\frac{T_{1}}{T_{2}}\right)^{b}
$$

where $k_{2}$ is the new thermal conductivity at temperature $T_{2}$ relative to thermal conductivity $k_{1}$ at temperature $T_{1}$, and $b=1$. This scaling rule is true only for $T>\theta_{\mathrm{D}}$. To test the validity of this rule, we have tried fitting Equation (40) to the undoped and 4 at. \% and 15 at. \% Yb data of [29]. The results are shown in Figure 7. For undoped YAG we have found that the best fit to the data is $b=1.315$, a significant departure from Equation (40) with $b=1$. For the 4 at. $\% \mathrm{Yb}$ data, however, we find $b=1$, and for the 15 at. $\% \mathrm{Yb}$ data, $b=0.8$. It is clear from this data that the exponent $\mathrm{b}$ in Equation (38) is significantly different than 1 , a likely consequence of modeling data in the temperature range of about 100-300 K, whilst using a model that is strictly valid for $T \gg \theta_{\mathrm{D}}$. The decreased exponent for the doped cases, with $\mathrm{b}$ decreasing as doping is increased, is very likely due to the change in the Debye temperature as doping increases. Similar results may be obtained by using an exponential rather than Equation (40) to fit the data.

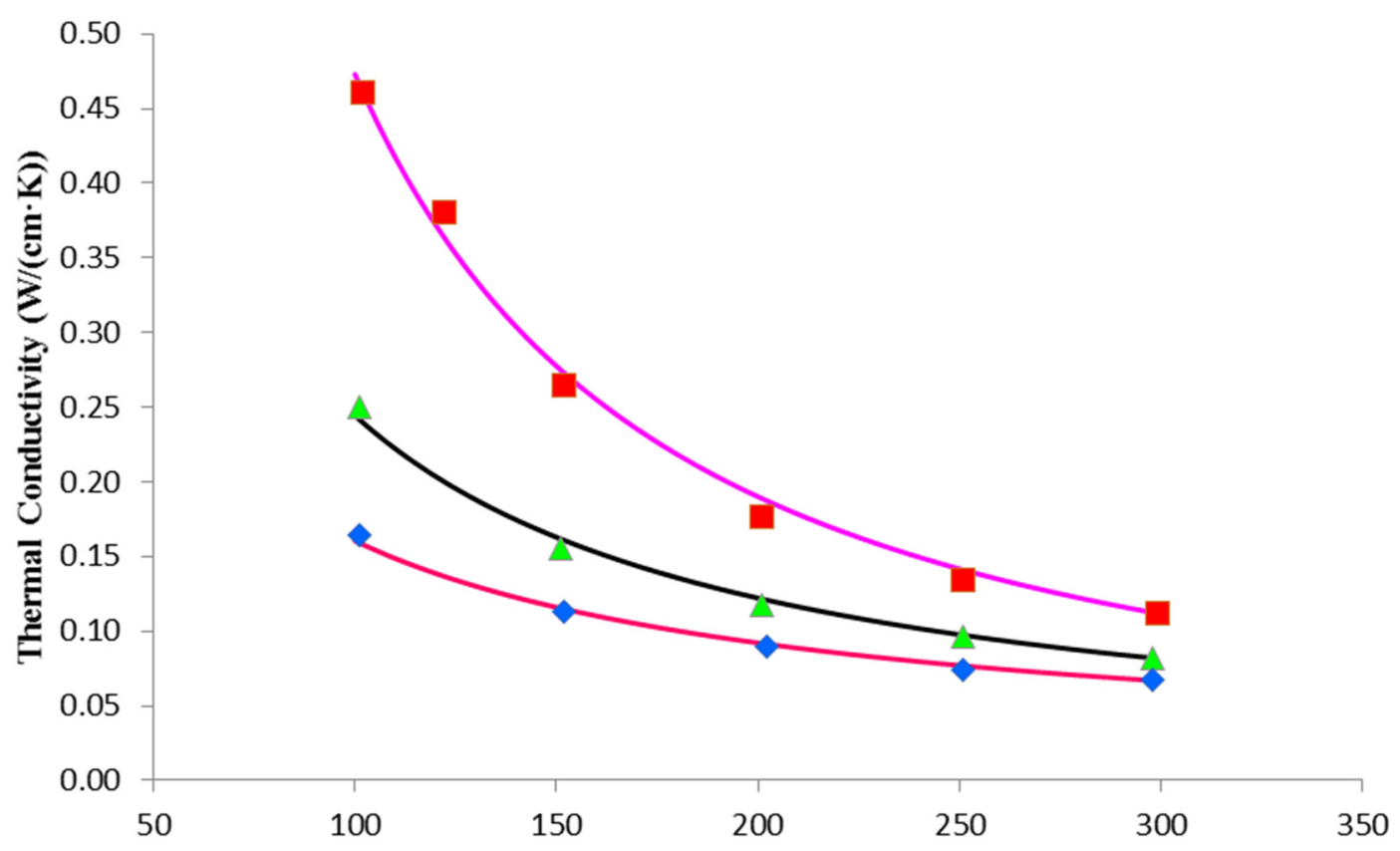

\section{Absolute Temperature (K)}

Figure 7. Thermal conductivity of undoped YAG (red), 4.0 (green) and 15.0 (blue) at. \% Yb-doped YAG as a function of absolute temperature. Data are from [29]; also shown are fits to the data showing that the undoped case scales as $b=1.315$ (pink), the 4.0 at. \% Yb case (black) scales as $b=1$, and the 15 at. $\% \mathrm{Yb}$ case (orange) scales as $b=0.8$.

\subsubsection{Modeling Thermal Conductivity}

A number of models have been developed to describe the variation in thermal conductivity as dopant concentration increases. One of the most successful is due to Klemens [42]. Other contributions 
were made by Slack and Oliver [45] and Holland [46]. A similar model was discussed in [47]. We follow the treatment of Klemens [42], who defines two relaxation times $\tau_{U}$ and $\tau_{\mathrm{D}}$ as

$$
\frac{1}{\tau_{U}}=B \omega^{2}, \frac{1}{\tau_{D}}=A \omega^{4}
$$

The relaxation time $\tau_{U}$ is associated with Umklapp anharmonic three-phonon scattering, while $\tau_{D}$ is the relaxation time for acoustic phonon scattering from dopants or impurities. The equation for $\tau_{D}$ is analagous to the Rayleigh scattering of optical photons, which has the same frequency dependence. The quantity $A$ is a constant that depends on the mass difference between donor and acceptor ions, the unit cell volume, and the phonon speed. $A$ can formally be written

$$
A=\frac{a^{3}}{4 \pi v_{s}^{3}} \cdot \delta
$$

In Equation (42), a is the unit cell lattice parameter, $v_{s}$ the speed of sound in the crystal, and $\delta$ is the mass-mismatch variance, given by

$$
\delta=\sum_{i} x_{i}\left[\frac{M_{i}-M}{M}\right]^{2}
$$

and $M$ is

$$
M=\sum_{i} x_{i} M_{i}
$$

The quantity $x_{i}$ is the concentration parameter of the donor atoms, and satisfies the inequality $0 \leqslant x_{i} \leqslant 1$. B in Equation (39) is a constant that is proportional to the temperature. For Umklapp and dopant scattering together, the effective phonon relaxation time $\tau_{e}$ is calculated using Matthiessen's rule:

$$
\frac{1}{\tau_{e}}=\frac{1}{\tau_{U}}+\frac{1}{\tau_{D}}
$$

Substituting in Equation (39), and using Equation (41), we find

$$
k=\frac{k_{B}}{2 \pi^{2} v_{S}} \cdot \int_{0}^{\omega_{D}} \frac{\omega^{2}}{\frac{1}{\tau_{U}}+\frac{1}{\tau_{D}}} d \omega=\frac{k_{B}}{2 \pi^{2} v_{S}} \cdot \int_{0}^{\omega_{D}} \frac{\omega^{2}}{B \omega^{2}+A \omega^{4}} d \omega
$$

We can define a characteristic frequency $\omega_{0}$ using the condition $\tau_{U}\left(\omega_{0}\right)=\tau_{D}\left(\omega_{0}\right)$ where the impurity scattering time constant is equal to the intrinsic Umklapp time constant. This condition can be expressed as, using Equation (41):

$$
\frac{1}{B \omega_{0}^{2}}=\frac{1}{A \omega_{0}^{4}}
$$

This may be re-arranged to yield

$$
\omega_{0}=\sqrt{\frac{B}{A}}
$$

Equation (46) may be simplified to give

$$
k=\frac{k_{B}}{2 \pi^{2} v_{S} B} \cdot \int_{0}^{\omega_{D}} \frac{1}{1+\left(\frac{A}{B}\right) \omega^{2}} d \omega
$$


The integral is Equation (49) may be solved analytically, yielding

$$
k=\frac{k_{B} \omega_{0}}{2 \pi^{2} v_{s} B} \cdot \tan ^{-1}\left(\frac{\omega_{D}}{\omega_{0}}\right)
$$

We now define the intrinsic thermal conductivity $k_{0}$ by setting $A=0$ in Equation (47), and taking $k=k_{0}$, the following results:

$$
k_{0}=\frac{k_{B} \omega_{D}}{2 \pi^{2} v_{S} B}
$$

Dividing Equation (50) by Equation (51), we obtain the result

$$
k=k_{0} \cdot\left(\frac{\omega_{0}}{\omega_{D}}\right) \cdot \tan ^{-1}\left(\frac{\omega_{D}}{\omega_{0}}\right)
$$

By use of Equation (39) and with $B$ being proportional to the temperature $T$, we can use Equation (48) to get

$$
\omega_{0}=K \sqrt{\frac{T}{\delta}}
$$

where $K$ is a proportionality constant. We then define a constant $\xi$ as

$$
\xi=\frac{\omega_{D}}{K}
$$

so that $\omega_{0} / \omega_{D}$ is expressed as

$$
\frac{\omega_{0}}{\omega_{D}}=\frac{1}{\xi} \sqrt{\frac{T}{\delta}}
$$

Substituting Equation (55) into Equation (52) gives the final result:

$$
k=k_{0} \cdot\left(\frac{1}{\xi} \sqrt{\frac{T}{\delta}}\right) \cdot \tan ^{-1}\left(\xi \sqrt{\frac{\delta}{T}}\right)
$$

Knowing $k_{0}, T$, and $\delta$, we can now calculate the thermal conductivity using $\xi$ as a fitting constant. Turning our attention to the binary laser crystal system YAG and YbAG, we can use Vegard's Law for solid solution to write the intrinsic thermal conductivity as

$$
k_{0}=k_{0}^{\mathrm{Y}}(1-x)+k_{0}^{\mathrm{Yb}} x
$$

whereas before $x$ is the concentration parameter for $\mathrm{Yb}$, and $k_{0}^{\mathrm{Y}}$ and $k_{0}^{\mathrm{Yb}}$ are the zero-doping intrinsic thermal conductivities of YAG and YbAG respectively.

To obtain $k$ as a function of $\mathrm{Yb}$ doping density, at any temperature, we then use the following relationship:

$$
k=\left[k_{0}^{Y}(1-x)+k_{0}^{Y b} x\right] \cdot\left(\frac{1}{\xi} \sqrt{\frac{T}{\delta}}\right) \cdot \tan ^{-1}\left(\xi \sqrt{\frac{\delta}{T}}\right)
$$

This model has been previously discussed in the Dissertations of Klopp and Fredrich-Thornton [48,49].

At a temperature of $300 \mathrm{~K}$, and using the intrinsic thermal conductivities of YAG and YbAG, $0.111 \mathrm{~W} /(\mathrm{cm} \cdot \mathrm{K})$ and $0.070 \mathrm{~W} /(\mathrm{cm} \cdot \mathrm{K})$, we obtained the results shown in Figure 8, where the thermal conductivity is displayed as a function of $x$, using the value $\xi=100$, that visually best fits the experimental data, obtained from the summary provided in [49]. To simplify the presentation, we averaged the many values of [49] for YAG $(x=0)$. Undoubtedly, a nonlinear least squares fit of Equation (56) to the experimental data would allow a more accurate determination of $\xi$, however, for reasons discussed in the next paragraph we have reservations as to the wisdom of such an undertaking. 


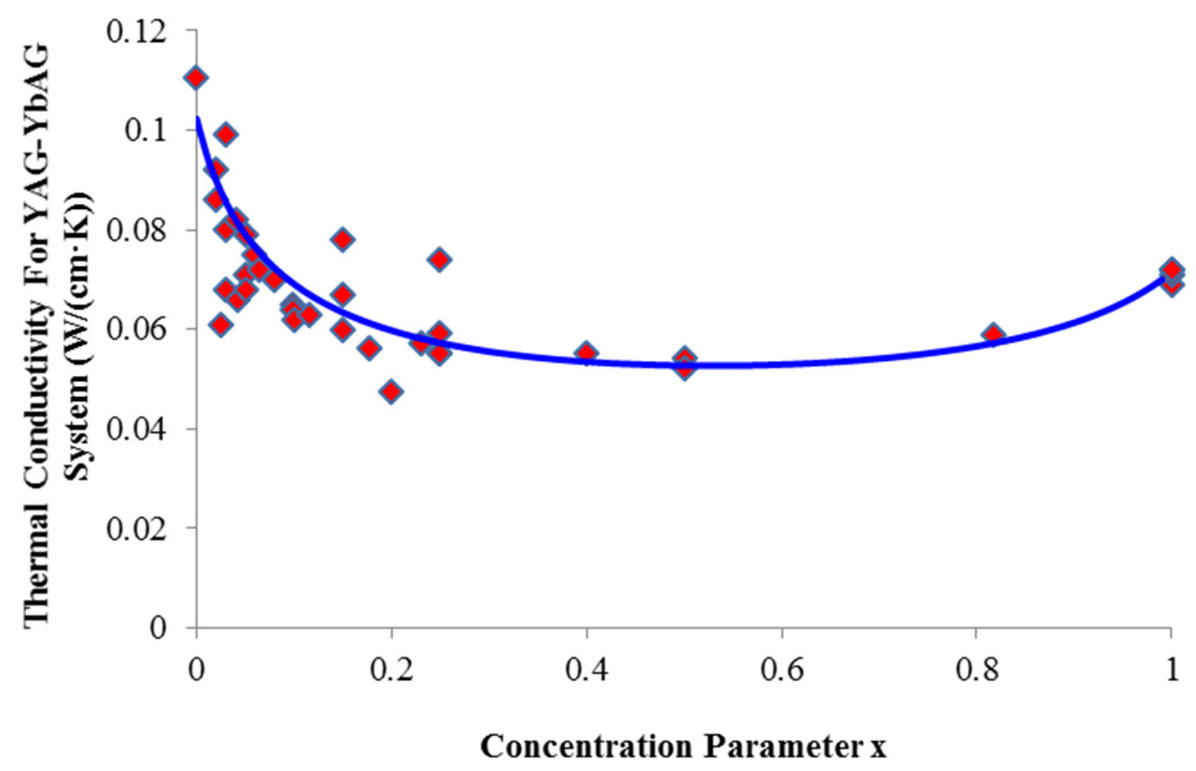

Figure 8. Thermal conductivity calculated using the Klemens model [42] as a function of concentration parameter $x$ for the binary solid solution YAG-YbAG. Data points are taken from the comprehensive summary found in [49].

While this model qualitatively explains the concentration dependence of $k$, and of course because of Equation (57) duplicates the two end point thermal conductivities for YAG and LuAG, it is very likely that the appearance of accurately fitting the experimental data is illusory, because such a curve requires more than one constant to be theoretically duplicated. Another aspect of the Klemens model that is disquieting is that it treats the case where $\mathrm{Y}$ acoustic phonons are scattered as $\mathrm{Yb}$ ions are doped into the crystal, where the $\mathrm{Yb}$ concentration varies from $0 \%-100 \%$, but ignores the reciprocal scattering of $\mathrm{Yb}$ ions from $\mathrm{Y}$ ions when $\mathrm{Y}$ is substituted into $\mathrm{YbAG}$. Also, it is clear that beyond $\mathrm{a} \mathrm{Yb}$ doping concentration of $50 \%$, the crystal is predominantly YbAG rather than YAG. It should also be pointed out that YAG and YbAG have significantly different Debye temperatures of 760 and $575 \mathrm{~K}$ respectively [40]. Because of the interaction between the two crystal lattices, it is unlikely that a Vegard's law linear relationship exists that can be used to estimate the values of the Debye temperature for $x$ values in the range $0<x<1$. The Klemens model uses only a single Debye temperature to describe the entire range of dopings, and for simplicity makes the assumption that one is operating at the high temperature limit where $T \gg \theta_{D}$, well beyond the region of interest for room temperature and cryogenic lasers. We will discuss progress towards an alternative model in the following section.

\subsubsection{Extended Thermal Conductivity Model}

Using the formalism shown in Section 2.2.5, we have developed an alternative model that splits the thermal conductivity into two components, $k_{\mathrm{Y}}(x)$ and $k_{\mathrm{Yb}}(x)$ whose properties are somewhat more physical, is symmetric with respect to the interchange of $\mathrm{Y}$ and $\mathrm{Yb}$, and that has the reasonable boundary conditions that as $x \rightarrow 0, k_{\mathrm{Yb}}(x) \rightarrow 0$, and for $x \rightarrow 1, k_{\mathrm{Y}}(x) \rightarrow 0$. The thermal conductivity for any value of the doping parameter $x$ can then be written as the equations

$$
k=(1-x) k_{\mathrm{Y}}+x k_{\mathrm{Yb}}
$$

and

$$
\begin{aligned}
k(x)=(1-x) \cdot\left(\frac{k_{o Y}}{\xi_{Y}}\right) & \cdot \sqrt{\frac{T}{\delta_{Y}}} \cdot \tan ^{-1}\left(\xi_{Y} \cdot \sqrt{\frac{\delta_{Y}}{T}}\right)+x \cdot\left(\frac{k_{o Y b}}{\xi_{Y b}}\right) \cdot \sqrt{\frac{T}{\delta_{Y b}}} \\
& \cdot \tan ^{-1}\left(\xi_{Y b} \cdot \sqrt{\frac{\delta_{Y b}}{T}}\right)
\end{aligned}
$$


These equations satisfy the aforementioned boundary conditions, are symmetric upon the interchange of $\mathrm{Y}$ and $\mathrm{Yb}$, and are physically reasonable since now a crystal may be viewed as a solid solution of two separate scattering materials rather than a single crystal with impurity doping added, and there are now two constants $\xi_{Y}$ and $\xi_{Y b}$ that may be adjusted to fit the experimental data. The quantities $k_{\mathrm{O}}$ and $k_{\mathrm{O} \mathrm{Yb}}$ are the experimental values of the thermal conductivity of YAG and YbAG. The mass scattering terms $\delta_{Y}$ and $\delta_{Y b}$ are now given by the expressions

$$
\delta_{Y}=(1-x) \cdot\left[\frac{\left(M_{Y}-M\right)}{M}\right]^{2} ; \delta_{Y b}=x \cdot\left[\frac{\left(M_{Y b}-M\right)}{M}\right]^{2}
$$

where $M$ is given by Equation (44), and $M_{Y}$ and $M_{Y \mathrm{~b}}$ are the atomic masses of $Y$ and $Y b$ respectively.

In Figure 9, we shown a fit to the experimental data summarized in [49]. The thermal conductivity $k_{\mathrm{Y}}$ goes to 0 for $x=1$ and for $x=0, k_{\mathrm{Yb}}=0$, as expected. The minimum appears to be closer to $x=0.35$ than to $x=0.5$ as found in the previous plot, Figure 8 , but without a better more consistent data set, it is difficult to ascertain exactly where it is with any accuracy. The position of the minimum is determined by how quickly the thermal conductivities decrease as a function of $x$. The equality of the two thermal conductivities occurs at $x=0.4$.

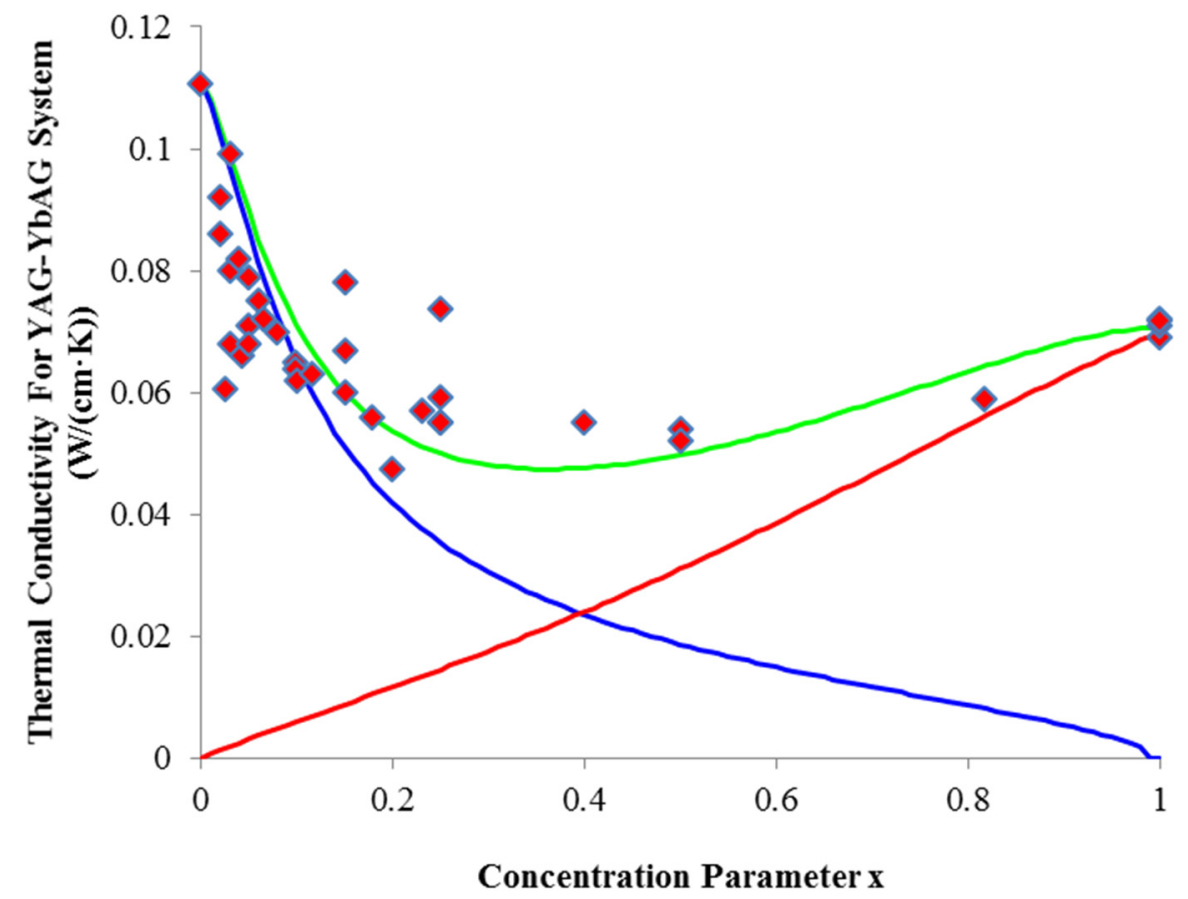

Figure 9. Thermal conductivities $k_{\mathrm{Y}}$ (blue), $k_{\mathrm{Yb}}$ (red), and $k$ (green) calculated using our model as a function of concentration parameter $x$ for the binary solid solution YAG-YbAG. Data points are taken from the comprehensive summary found in [49]. Constants used in this plot are $\xi_{Y}=300$ and $\xi_{Y b}=50$.

This new model seems to offer more flexibility with regard to fitting experimental data, however we view this as only a first step in developing a more comprehensive model that can be used to predict in advance the thermal conductivities of arbitrary laser crystals. Because the Klemens model that we started with is only valid for $T>>\theta_{D}$, a reasonable next step is to combine the approach described here with Equation (36), and to solve for the thermal conductivity without approximation, using numerical methods. 
2.2.7. Other Reported Thermal Conductivity Measurements Including Commonly Used Ancillary Materials

Since our 2005 paper [2], the measurement of thermal conductivity values has accelerated, with most common laser materials being tabulated. In this section, we show plots of most of the data reported in the literature for single-crystal growth. Measurements for ceramic laser materials have not been included due to their well-known dependence on pore size. We showed previously (Figure 4) the thermal conductivity of YAG and Yb-doped YAG as a function of temperature and doping density (Figure 4), and for a number of undoped and $\mathrm{Yb}$-doped sesquioxide laser materials (Figures 5 and 6). Here we show similar plots for many other laser materials. We start with the biaxial laser material YALO $\left(\mathrm{YAlO}_{3}\right)$, where Figure 10 shows thermal conductivity as a function of absolute temperature for undoped and 5 at. $\% \mathrm{Yb}$ doped cases. Measurements of $\mathrm{k}$ were performed for the $a, b$, and $c$ crystal axes [29]. Due to the moderate mass mismatch between $\mathrm{Yb}$ and $\mathrm{Y}$ (Table 1), the $k$ values for 5 at. \% Yb doping are significantly less than the undoped values. Figure 11 shows $k$ values for undoped YAG and LuAG $[3,29,45]$, whilst Figure 12 plots the thermal conductivities of YAG and LuAG as a function of $\mathrm{Yb}$ doping density [50] and for undoped and $\mathrm{Tm}$-doped $\mathrm{Lu}_{2} \mathrm{O}_{3}$ [51]. These data show that for less than about 4 at. \% Yb Yb:YAG is superior while for greater than 4 at. \% Yb:LuAG is the better choice. The small change in $k$ for $\mathrm{Yb}$ :LuAG as doping density increases is due to the very small mass mismatch between $\mathrm{Yb}$ and $\mathrm{Lu}$. Similarly, for $\mathrm{Tm}: \mathrm{Lu}_{2} \mathrm{O}_{3}$, the mass mismatch between $\mathrm{Tm}$ and $\mathrm{Lu}$ is very small, and the thermal conductivity decreases very slowly with Tm concentration. Figure 13 plots $k$ as a function of absolute temperature for the uniaxial laser material YLF, for undoped and 5 at. \% and 25 at. \% doped $\mathrm{Yb}$ cases [29,30]. The large drop in $k$ for 5 and 25 at. \% $\mathrm{Yb}$ dopings is a consequence of the moderate mass mismatch between $\mathrm{Yb}$ and $\mathrm{Y}$. Figure 14 shows $\mathrm{k}$ for the recently developed undoped biaxial laser materials BYW, KGW, and KYW [29]. In Figure 15 we show the thermal conductivity of the important uniaxial ultrafast laser material $\mathrm{CaF}_{2}$, for undoped, 1 at. $\%, 3$ at. $\%$ and 5 at. \%, obtained from a number of sources [44,52-54]. It is important to point out that the very rapid drop in $\mathrm{k}$ for increased $\mathrm{Yb}$ doping density is partially due to the very large mass mismatch between $\mathrm{Ca}$ and $\mathrm{Yb}$ (see Table 1). According to [54], between about 1-3 at. \% Yb doping, where $k$ is almost flat from $100-300 \mathrm{~K}$, larger dopings drive the crystal structure towards clustering and increasingly glasslike behavior, resulting in $k$ values that decrease with decreasing temperature, rather than the increase in $k$ which we have seen is almost a universal phenomenon with pure crystalline materials. This type of behavior is also displayed by laser glasses, and other important optical materials often used in ultrafast laser systems. One reviewer of this paper has pointed out that mass-mismatch may play the lesser role in this case. Figure 16 for example, shows the thermal conductivity of fused silica $\left(\mathrm{SiO}_{2}\right)$ [55], an anaxial optical material, as a function of temperature. It displays a monotonic decrease in $k$ as temperature is lowered, as expected due to a locally disordered crystal structure that severely limits the phonon mean free path. In Figure 17 the thermal conductivity values for the $\mathrm{GdVO}_{4} c$ and $a$ axes, the YSO $b$-axis, and for GSAG are shown as a function of absolute temperature $[3,30]$. 


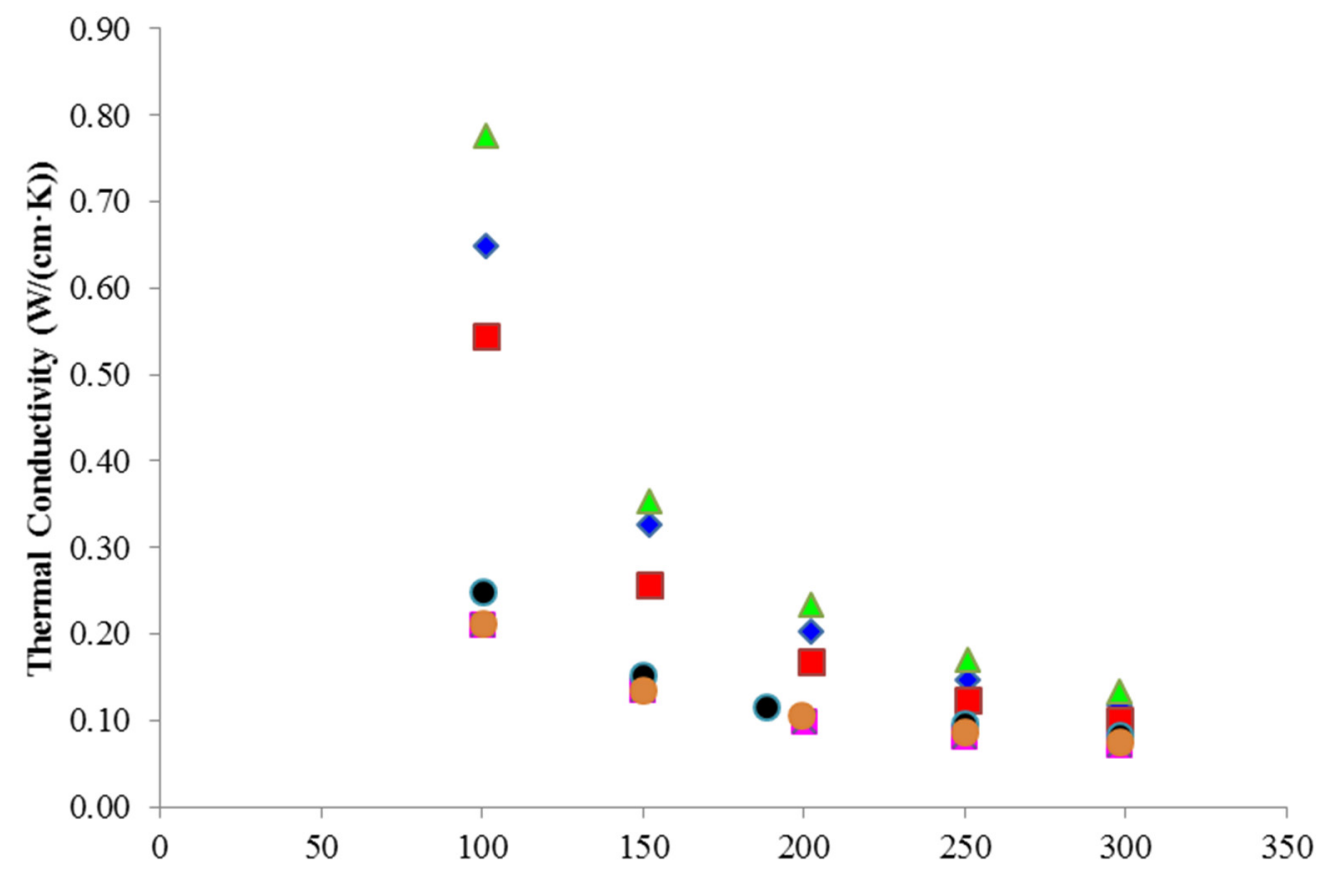

Absolute Temperature (K)

Figure 10. Thermal conductivity as a function of absolute temperature for the biaxial laser material YALO for undoped $a$ (blue), $b$ (red), and $c$ (green) axes and 5 at. \% Yb doped $a$ (pink), $b$ (black), and $c$ (orange) axes [29].

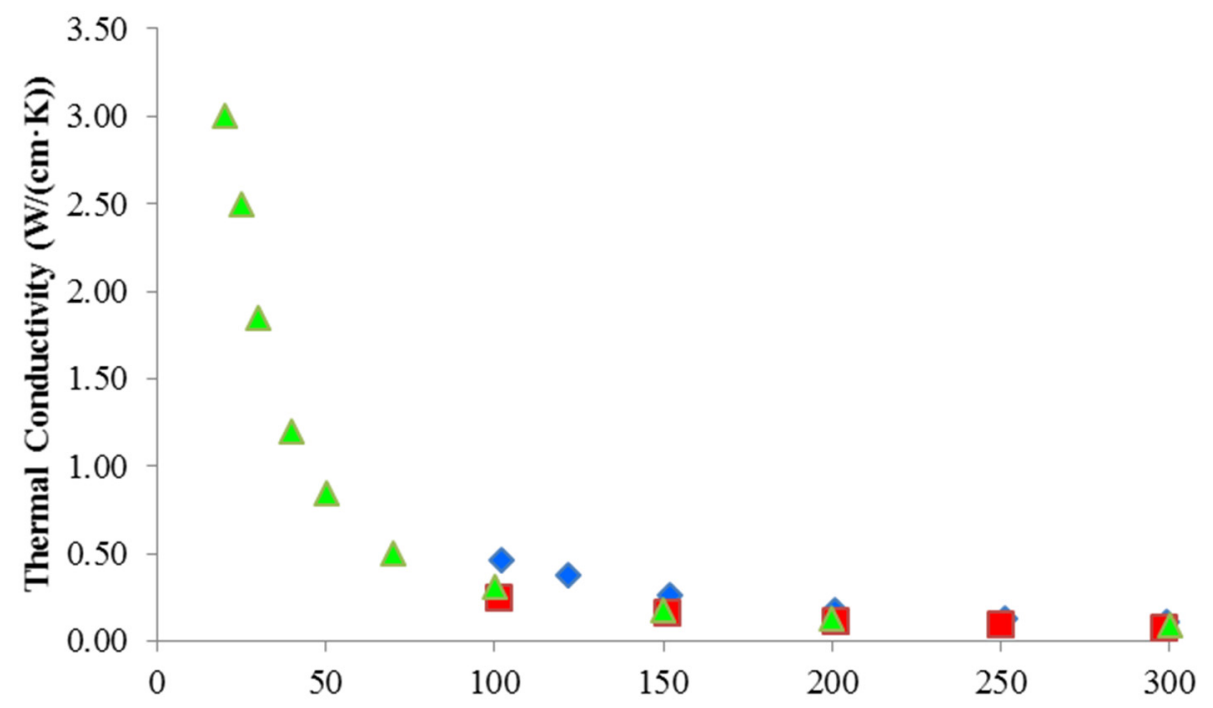

Absolute Temperature (K)

Figure 11. Thermal conductivity as a function of absolute temperature of the undoped anaxial laser materials LuAG (green) [45], YAG (blue) [3,29] and LuAG (red) [29]. 


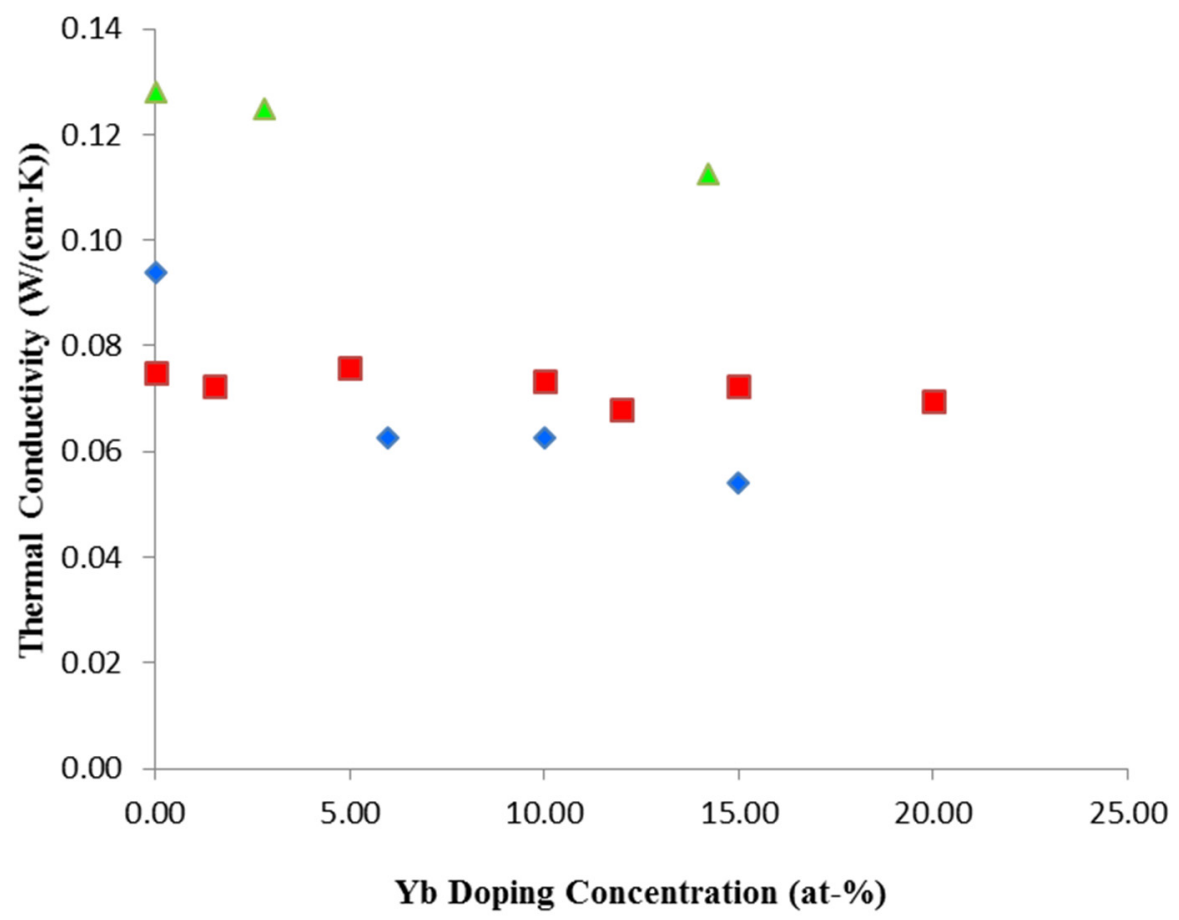

Figure 12. Thermal conductivity of the anaxial laser materials Yb:YAG (blue) [50], Yb:LuAG (red) [50], and $\mathrm{Tm}: \mathrm{Lu}_{2} \mathrm{O}_{3}$ (green) as a function of $\mathrm{Yb}$ doping concentration at room temperature [51].

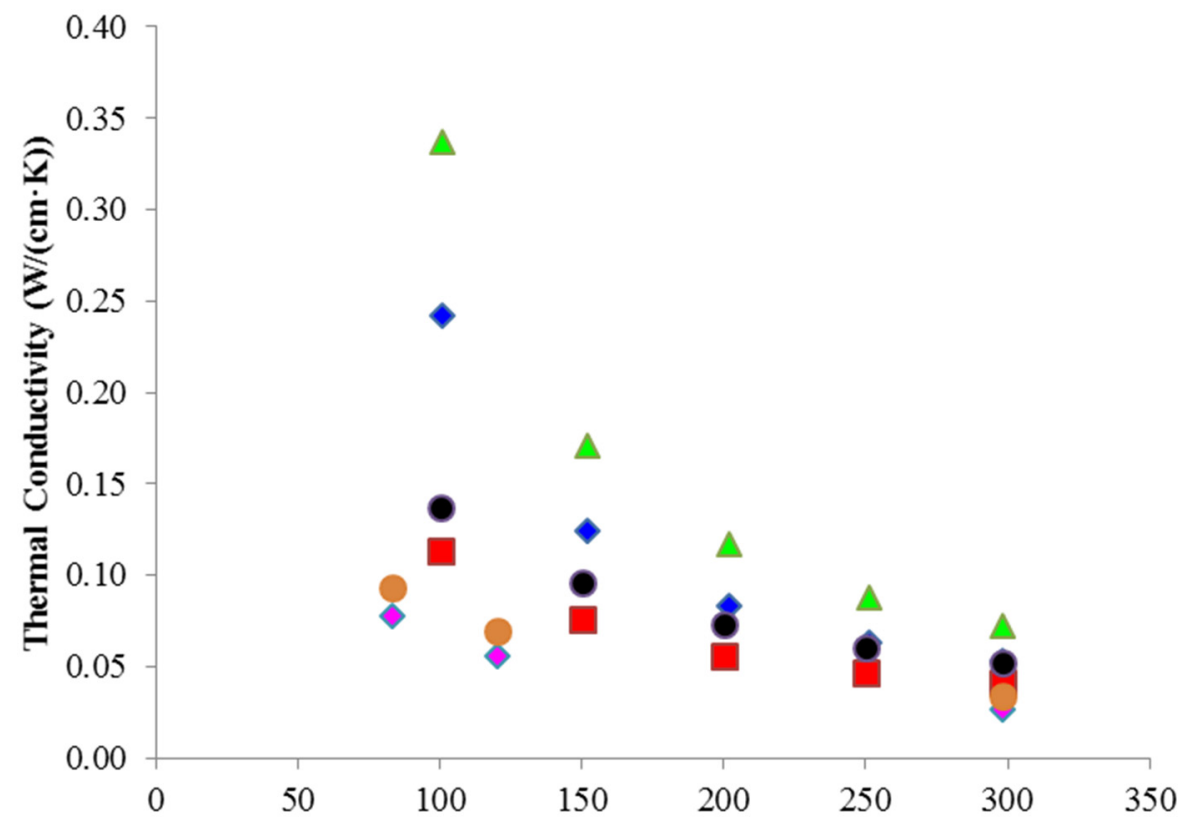

Absolute Temperature (K)

Figure 13. Thermal conductivity as a function of absolute temperature of the uniaxial laser material YLF for undoped, $a$ (blue) and $c$ axes (green), 5 at. \% doped, $a$ (red) and $c$ (black) axes, and 25 at. \% doped $a$ (pink) and $c$ axes (orange) $[29,30]$. 


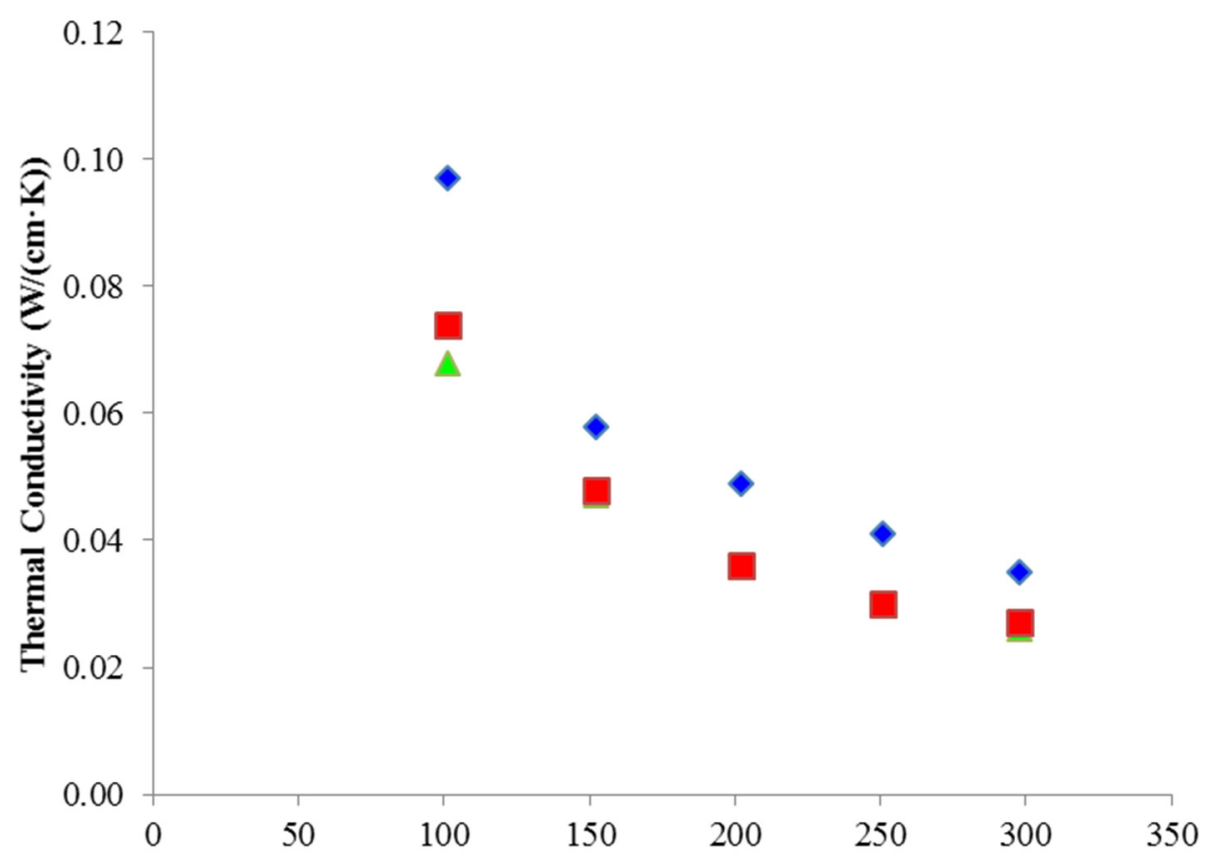

Absolute Temperature (K)

Figure 14. Thermal conductivity as a function of absolute temperature for the undoped biaxial laser materials BYW (blue), KGW (green), and KYW (red) along the $b$-axes [29].

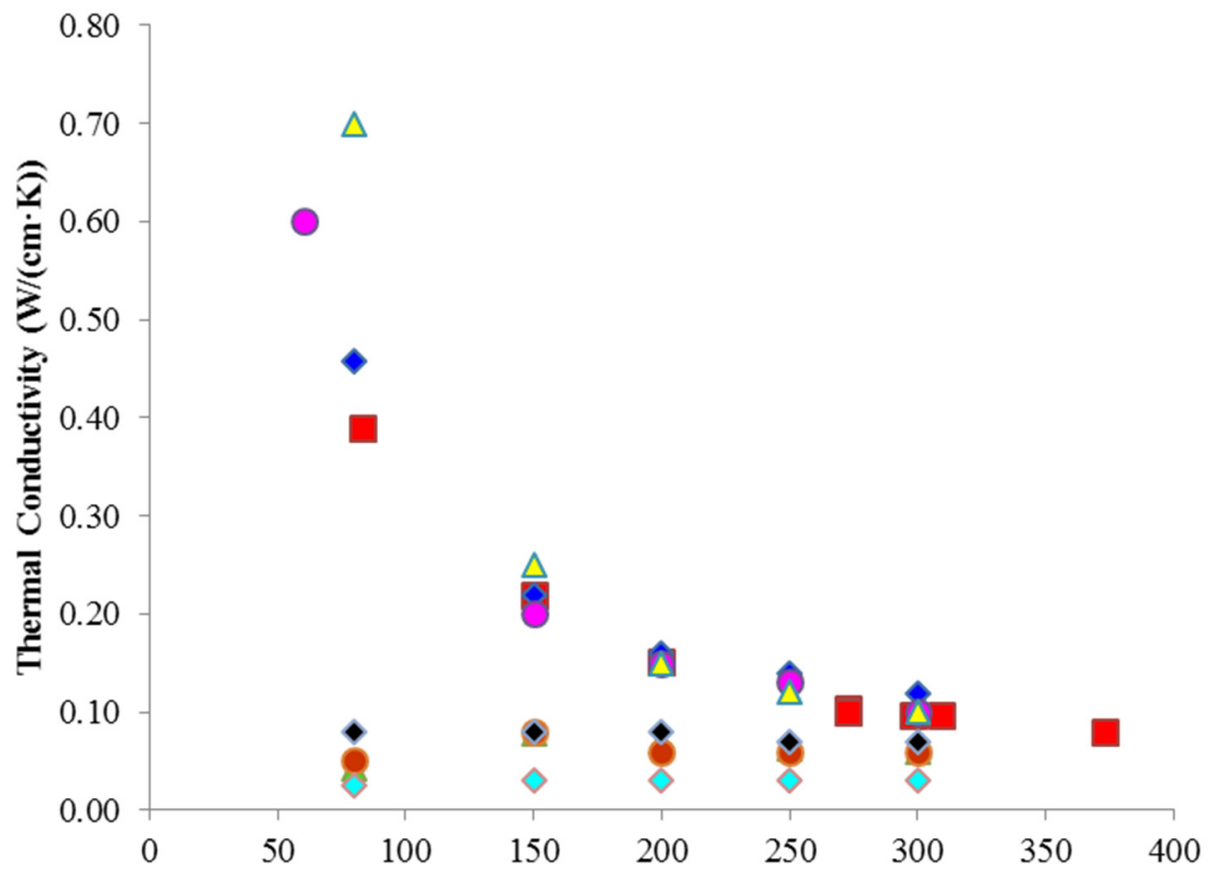

Absolute Temperature (K)

Figure 15. Thermal conductivity as a function of absolute temperature of the anaxial laser material $\mathrm{CaF}_{2}$, for undoped (red [52], blue [53], pink [45], yellow [54]), 1 (black [54]), 3 (orange [53], light blue [54]) and 5 (green [54]) at. \% doped $\mathrm{Yb}$ cases. 


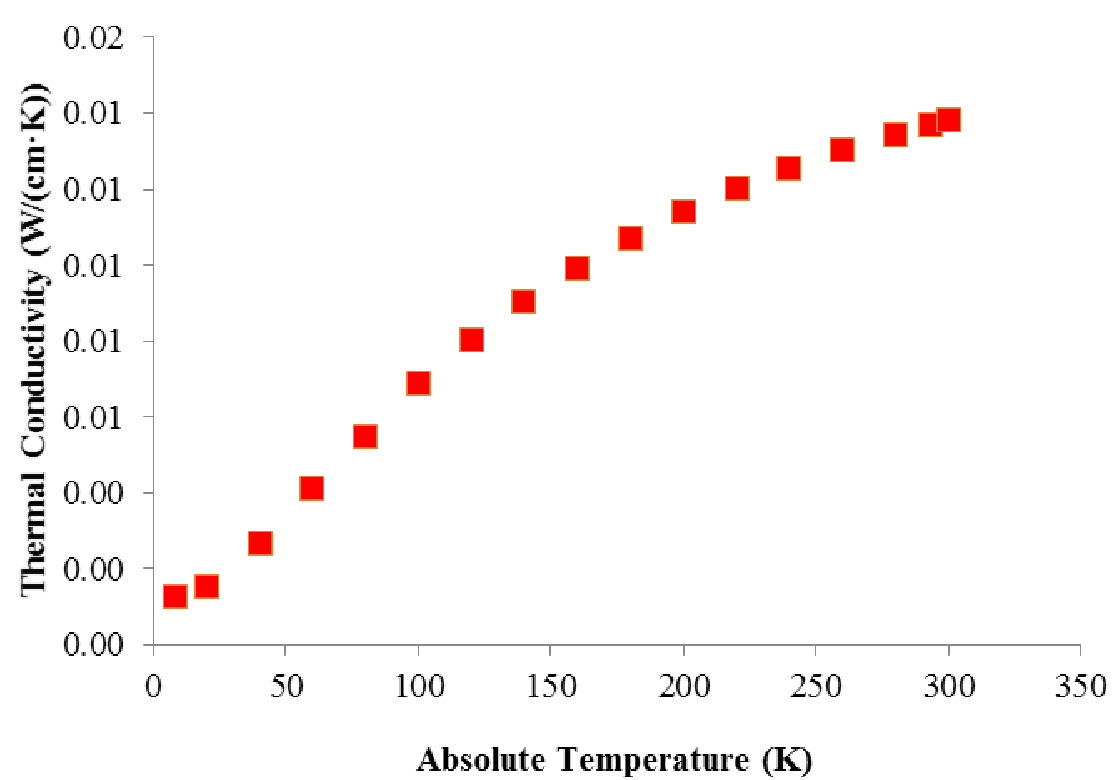

Figure 16. Thermal conductivity as a function of absolute temperature for the anaxial optical material $\mathrm{SiO}_{2}$ [55].

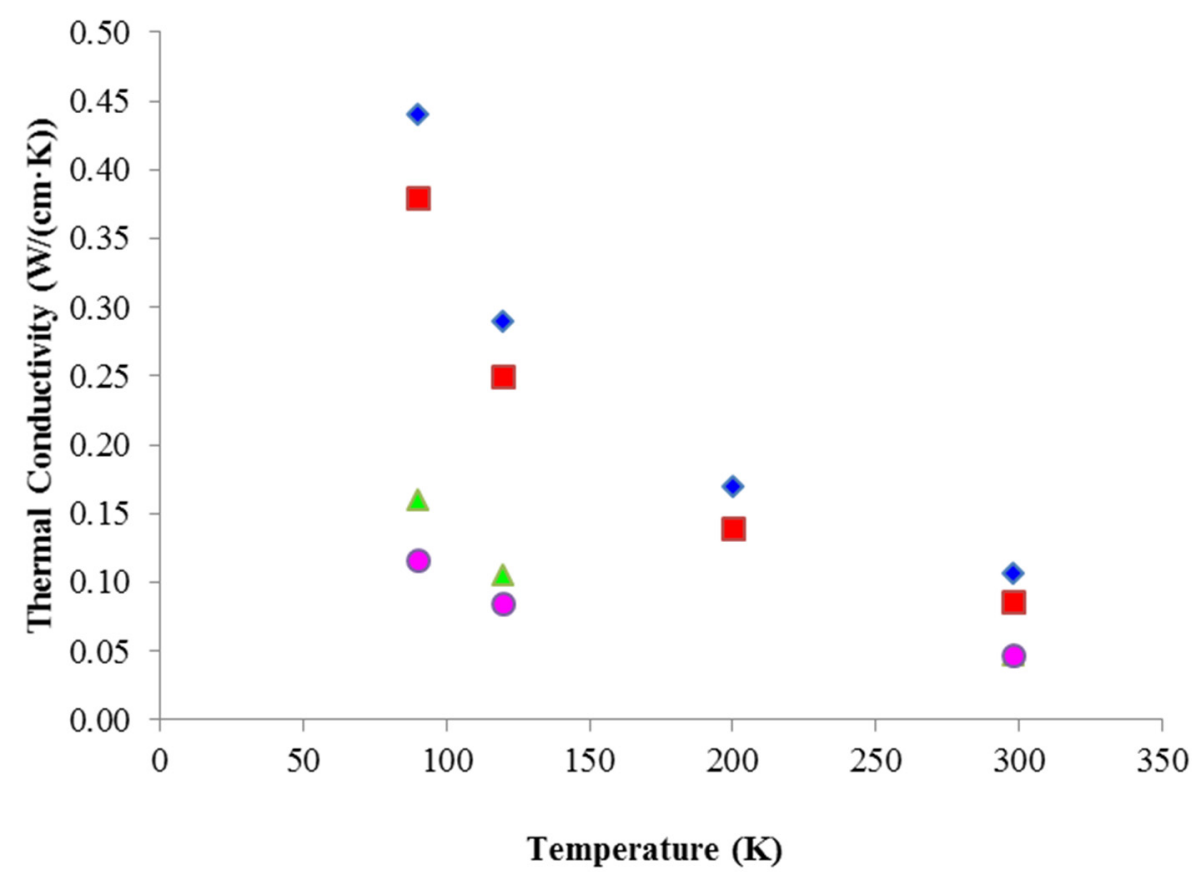

Figure 17. Thermal conductivity as a function of absolute temperature for the $a$ (red [3]) and $c$ (blue [3]) axes of the undoped uniaxial material $\mathrm{GdVO}_{4}$, the $b$-axis (green) of the biaxial material YSO [30], and the anaxial material GSAG (pink) [30].

In cryogenic laser systems the thermal conductivity of optical materials and metals used to construct pump chambers can be very important. For convenience, in Figures 18 and 19 we have plotted the thermal conductivities for the uniaxial optical material and laser material sapphire $\left(\mathrm{Al}_{2} \mathrm{O}_{3}\right)$ and for anaxial Type II a diamond, and for elemental copper, titanium, molybdenum, and aluminum respectively, as a function of absolute temperature. 


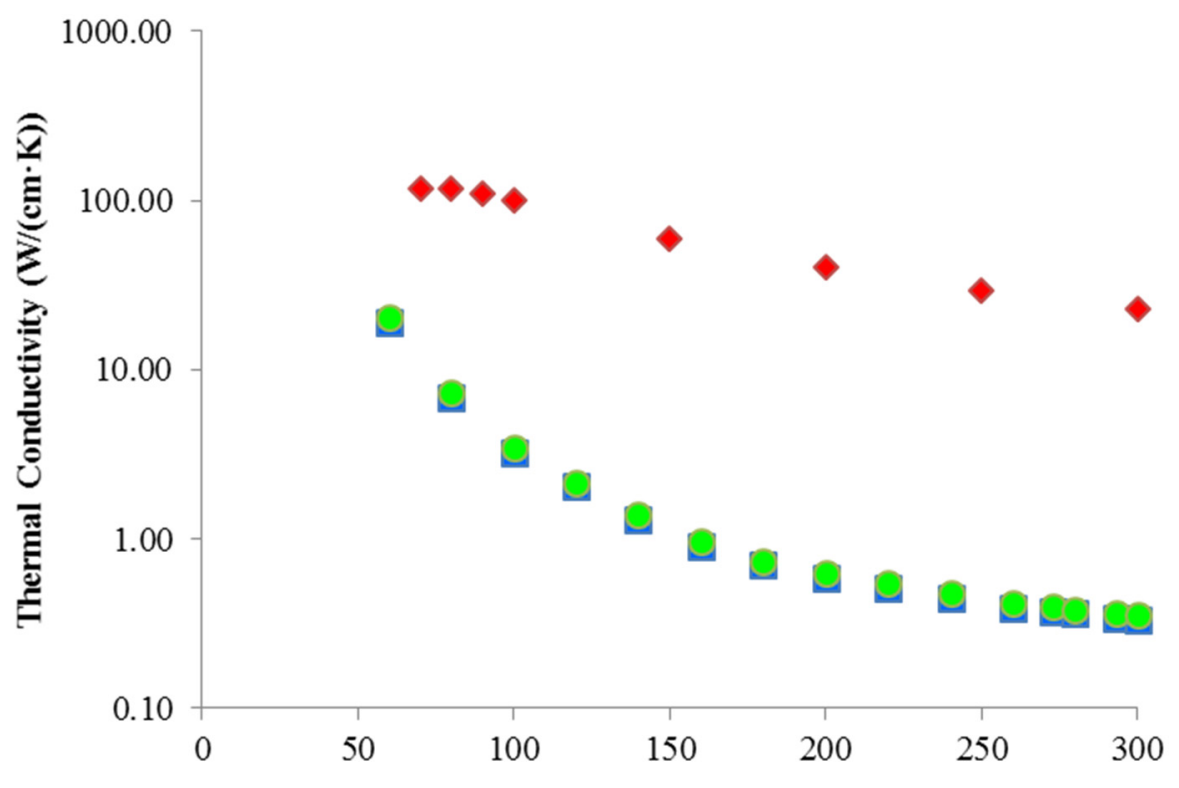

Absolute Temperature (K)

Figure 18. Thermal conductivity as a function of absolute temperature for the uniaxial optical material $\mathrm{Al}_{2} \mathrm{O}_{3} a$ (blue [55]) and $c$ (green [56]) axes and for Type II a isotropic diamond (red) [56].

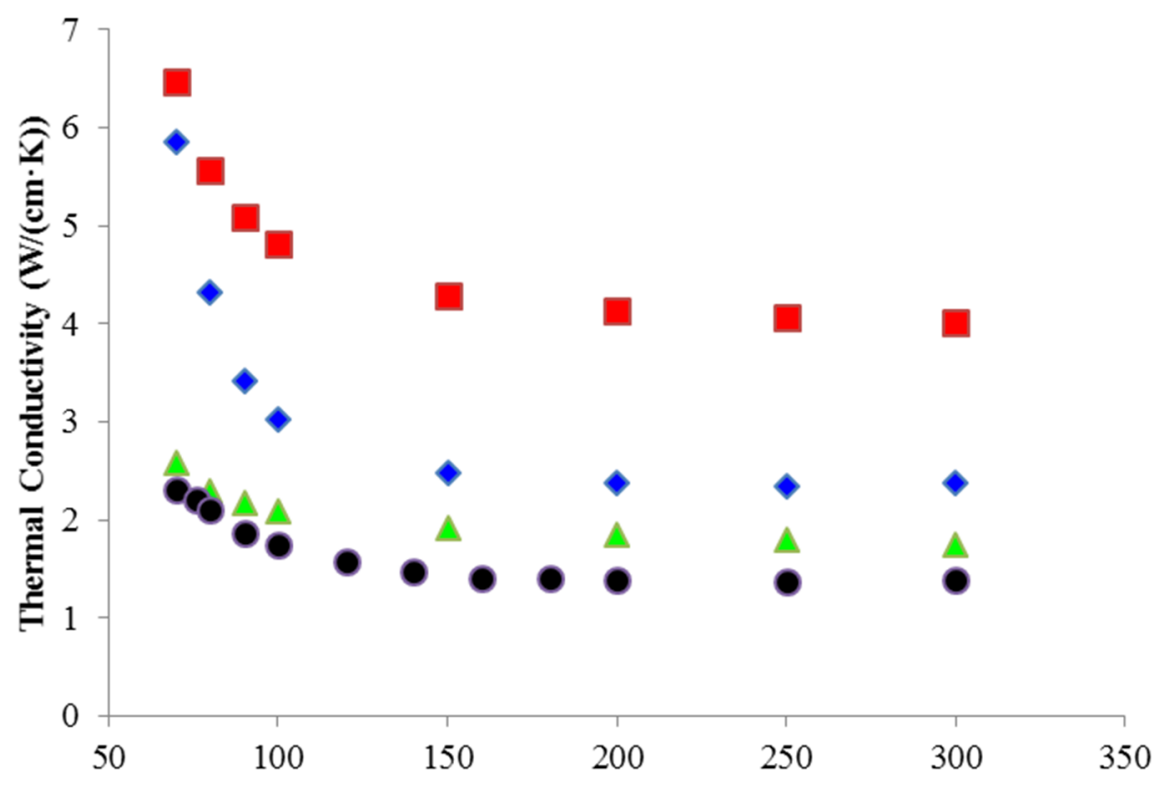

Absolute Temperature (K)

Figure 19. Thermal conductivity as a function of absolute temperature for the elemental metals copper (red [56]), titanium (green [56]), molybdenum (black [57]), and aluminum (blue [56]).

\subsection{Thermal Expansion Coefficient}

Thermal expansion of crystals can be understood by the realization that in the theory of lattice vibrations, thermal expansion only occurs when the potential energy of a classical harmonic oscillator is expanded to terms of higher order than two, since the quadratic dependence alone results in no net thermal expansion. For the thermodynamic volumetric thermal expansion coefficient $\alpha_{V}(T)$, it can be shown that the Grüneisen relationship applies to cubic or isotropic materials. This equation relates the 
Grüneisen constant $\gamma_{G}$, the bulk isothermal elastic modulus $B_{T}$ and the crystal mass density $\rho$ to the bulk thermal expansion coefficient according to

$$
\alpha_{V}=\frac{\rho C_{V}}{B_{T}} \cdot \gamma_{G}
$$

For isotropic laser materials, the linear thermal expansion $\alpha(T)$ coefficient is related to the bulk thermal expansion coefficient through:

$$
\alpha_{V}(T)=3 \cdot \alpha(T)
$$

Using Equations (62) and (63), we can also write the following relationship for the linear thermal expansion coefficient:

$$
\alpha(T)=\frac{1}{3} K_{T}(T) \gamma_{G} \rho(T) C_{V}(T)
$$

where we have used the relationship $K_{T}=1 / B_{T}$. Finally we can also use the well-known relation for isotropic materials:

$$
K_{T}=\frac{E}{2(1-v)}
$$

where $E$ is Young's modulus, and $v$ Poisson's ratio, to arrive at

$$
\alpha(T)=\frac{1}{6} \gamma_{G} \rho(T) C_{V}(T) \cdot \frac{E}{(1-v)}
$$

Because $E, v$, and $\rho$ are weak functions of $T, \alpha(T)$ is dominated by the strong functional dependence of $C_{V}(T)$ on $T$. This dependence can be seen in the thermal expansion coefficient data presented later in this section. Since theoretically $C_{v} \rightarrow 0$ as $T \rightarrow 0$, at absolute zero the thermal expansion coefficient is theoretically 0 also. While some data deviate from this simple rule, it is generally true that as temperature is lowered the thermal expansion coefficient decreases roughly with the temperature dependence of the specific heat. For simple cubic crystals like YAG, in the temperature range of interest in this paper, one may approximate the temperature dependence of $C_{V}$ using an exponential function:

$$
C_{V}(T)=C_{0} \exp \left(-\frac{\hbar \omega}{k_{B} T}\right)
$$

$C_{0}$ is a proportionality constant equal to

$$
C_{0}=\frac{1}{6} \frac{\gamma_{G} \rho E}{(1-v)}
$$

This approach has been taken in [58], where very good fits to thermal expansion data for YAG were obtained by fitting the proportionality constant $C_{0}$ to ceramic and crystalline data.

Reported Thermal Expansion Coefficient Measurements

In Figures 20-27 we display plots of thermo-optic coefficient as a function of absolute temperature for doped and undoped crystals. We have only included data sets available in the temperature region of interest in this paper, about $80-300 \mathrm{~K}$, and have not included any single-point measurements, most of which are available at or near $300 \mathrm{~K}$. Some data shown include ceramic laser materials since ceramic and single-crystal data are nearly indistinguishable. Except where discussed, all data shown are for undoped host laser materials, and we have used published fits to the thermal expansion data where available to generate the plots shown here, Figure 20 shows the thermal expansion coefficient as a function of absolute temperature over the range 100-300 $\mathrm{K}$ for the oxide laser materials YAG, LuAG, and YALO [29]. YAG and LuAG are both isotropic crystals whereas YALO is biaxial and 
therefore has three principal thermal expansion coefficients. In Figure 21 we show thermal expansion as a function of temperature for the fluoride materials YLF and LuLF [29], both uniaxial crystals with two principal axes each. Figure 22 shows thermal expansion data for the biaxial laser materials BYF, KYW, and KGW, along their b-axes [29]. Figure 23 displays thermal expansion data for the biaxial laser material YSO b-axis as well as for the isotropic material GSAG [30]. In Figure 24 data are shown for the uniaxial laser materials $\mathrm{YVO}_{4}$ and $\mathrm{GdVO}_{4}$ [3,30]. Thermal expansion coefficients as a function of absolute temperature for the undoped isotropic sesquioxide laser materials $\mathrm{Sc}_{2} \mathrm{O}_{3}$ [30]

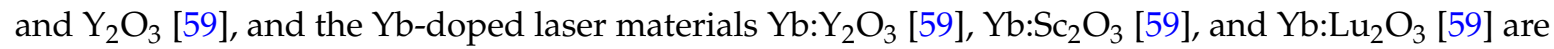
shown in Figure 25. Figure 26 shows thermal expansion data for the isotropic laser material $\mathrm{CaF}_{2}$, for undoped and 10 at. \% Yb doped crystals [59], while in Figure 27 we include some thermal expansion data for some other common laser and optical materials, uniaxial $\mathrm{Al}_{2} \mathrm{O}_{3}$, isotropic $\mathrm{MgO}$, and uniaxial $\mathrm{ZnO}[60]$.

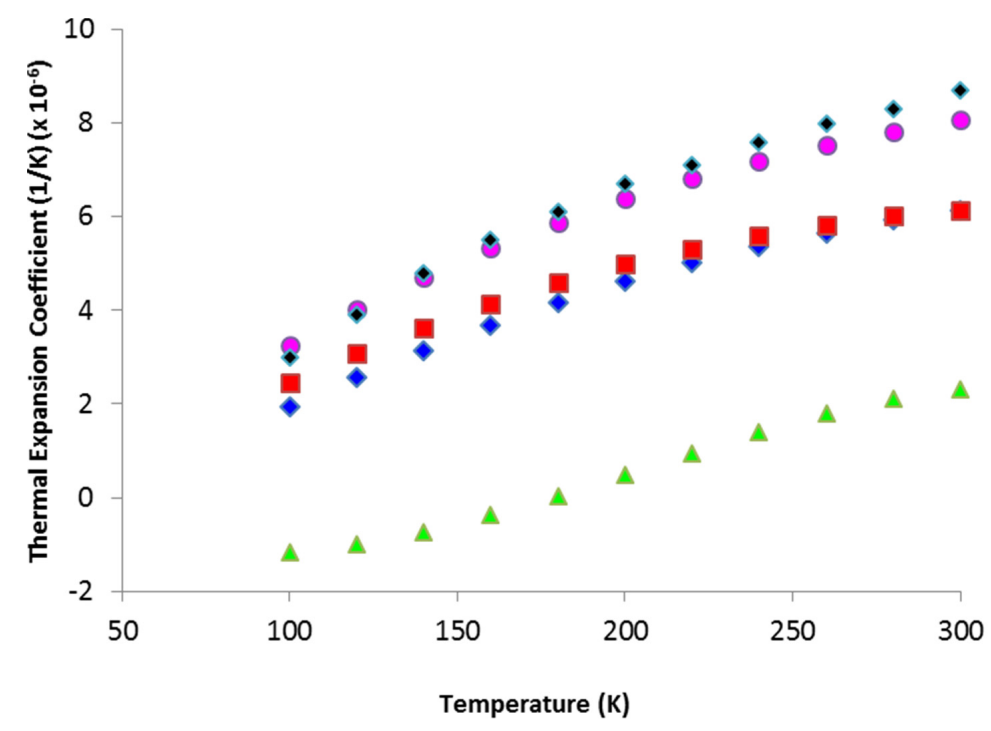

Figure 20. Thermal expansion coefficient as a function of absolute temperature for the isotropic laser materials YAG (blue) and LuAG (red) [29], and for the biaxial laser material YALO [29] along the $a$ (green), $b$ (pink), and $c$ (black) axes. All data are for undoped crystals.

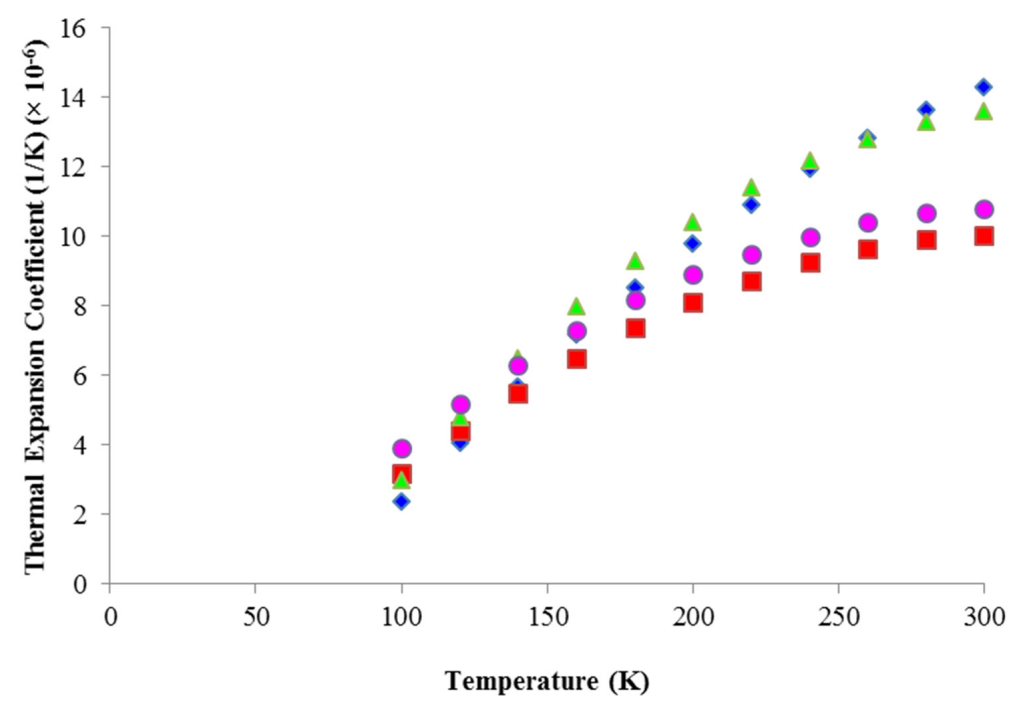

Figure 21. Thermal expansion coefficient as a function of absolute temperature for the uniaxial laser materials YLF along the $a$ (blue) and $c$ (red) axes [29] and LuLF along the $a$ (green) and $c$ (pink) axes [29]. All data are for undoped crystals. 


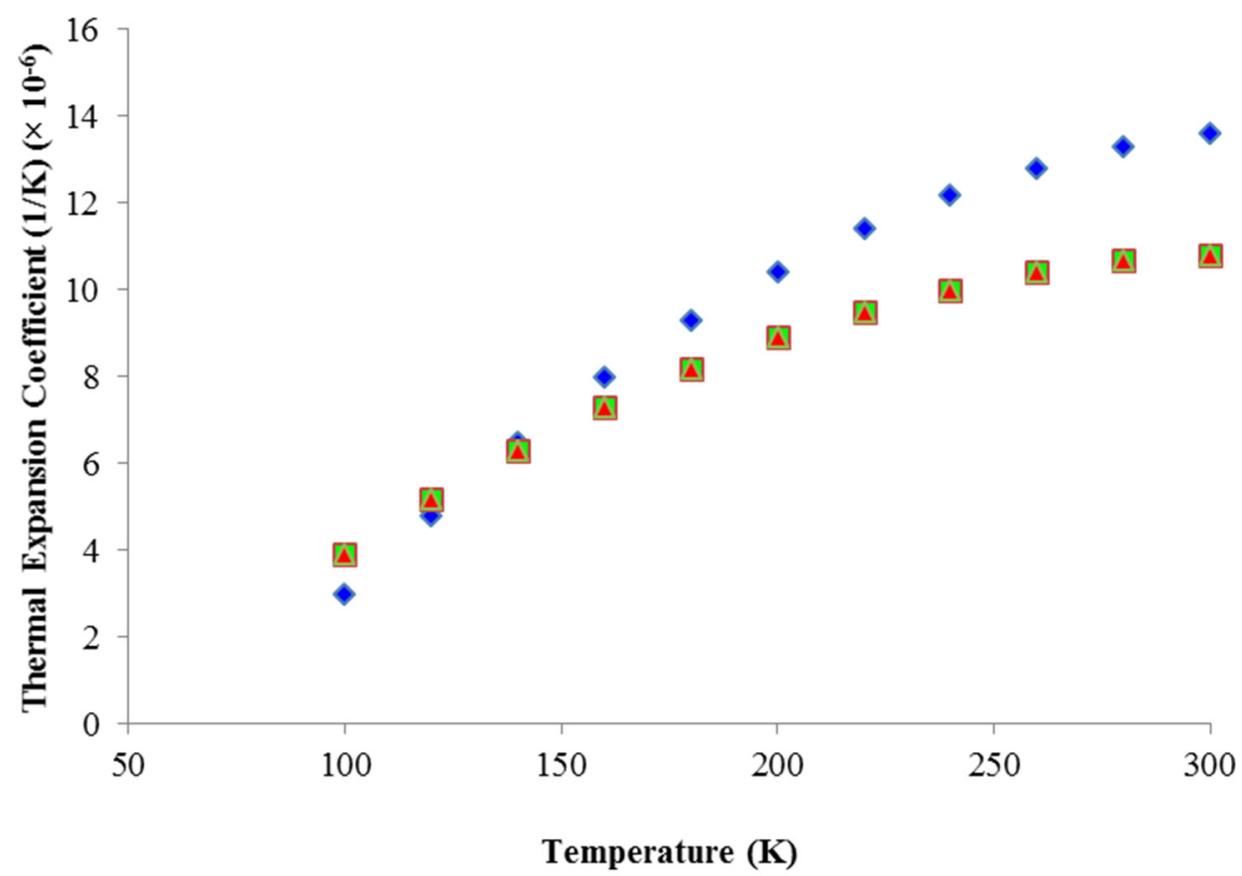

Figure 22. Thermal expansion coefficient as a function of absolute temperature for the biaxial laser materials BYF (blue), KGW (green), and KYW (red) [29]. All data are reported for the $b$-axis and for undoped crystals.

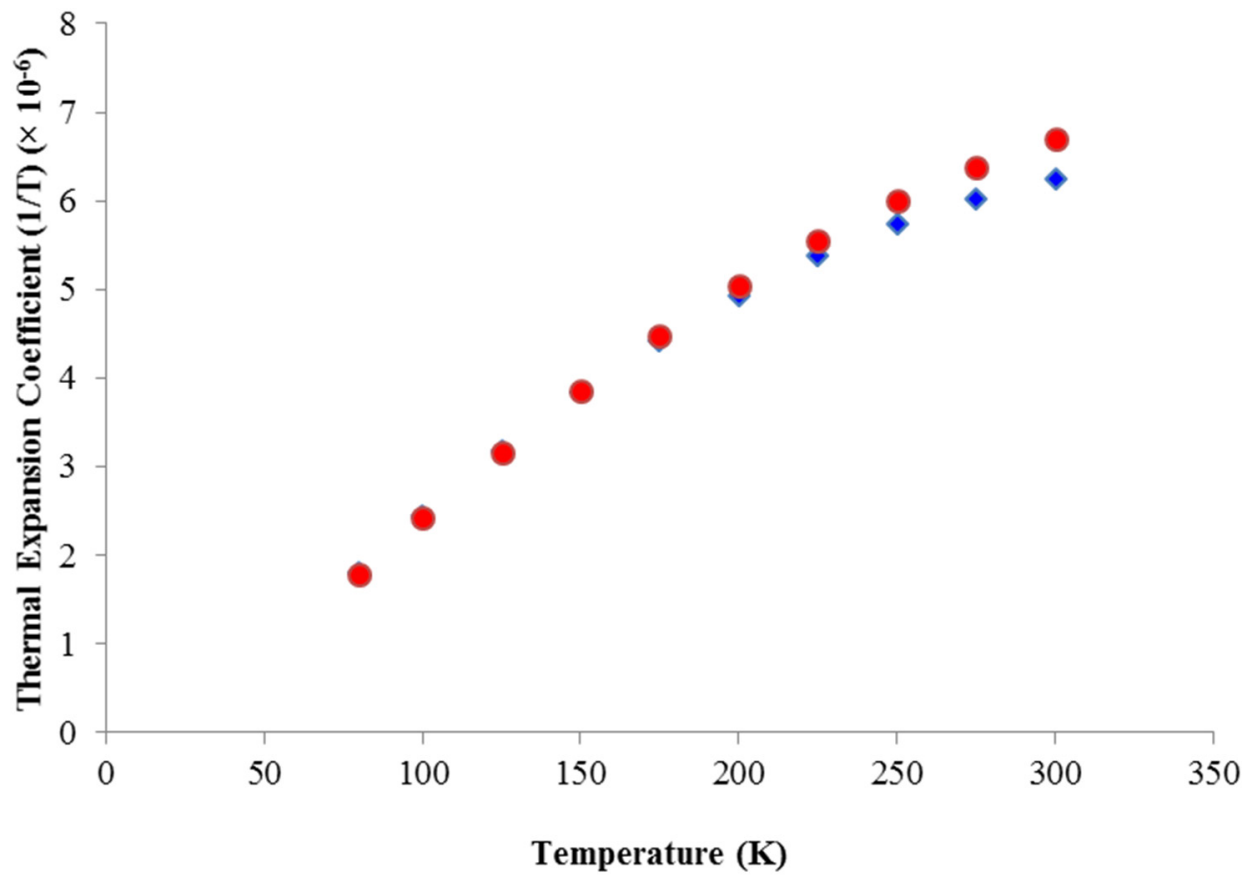

Figure 23. Thermal expansion coefficient as a function of absolute temperature for the biaxial laser material YSO (blue) [30] and the isotropic laser material GSAG (red) [30]. YSO data is reported for the $b$-axis, and all data are for undoped crystals. 


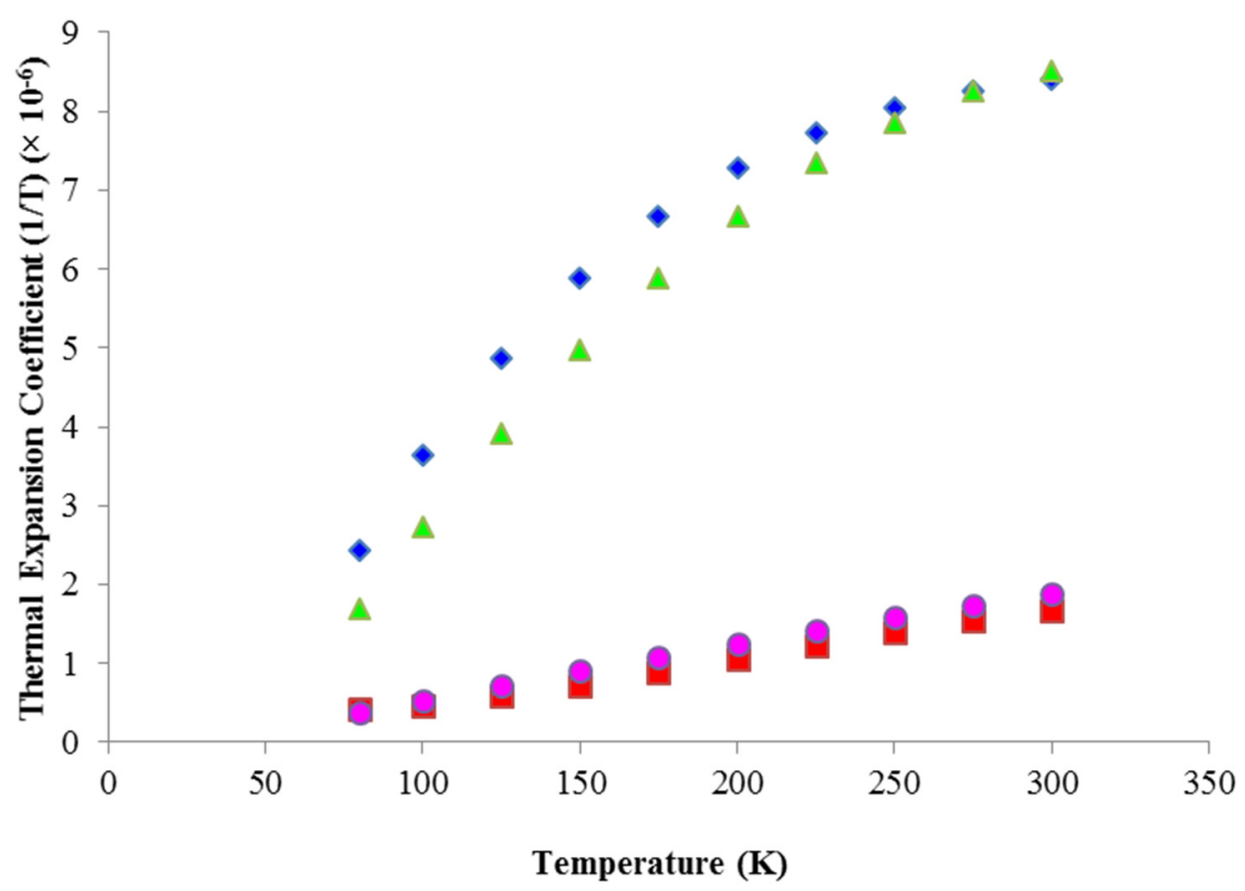

Figure 24. Thermal expansion coefficient as a function of absolute temperature for the uniaxial laser materials $\mathrm{YVO}_{4}$ [30] along the $a$ (pink) and $c$ (green) axes, and $\mathrm{GdVO}_{4}$ along the $a$ (red) and $c$ (blue) axes [3]. All data are for undoped crystals.

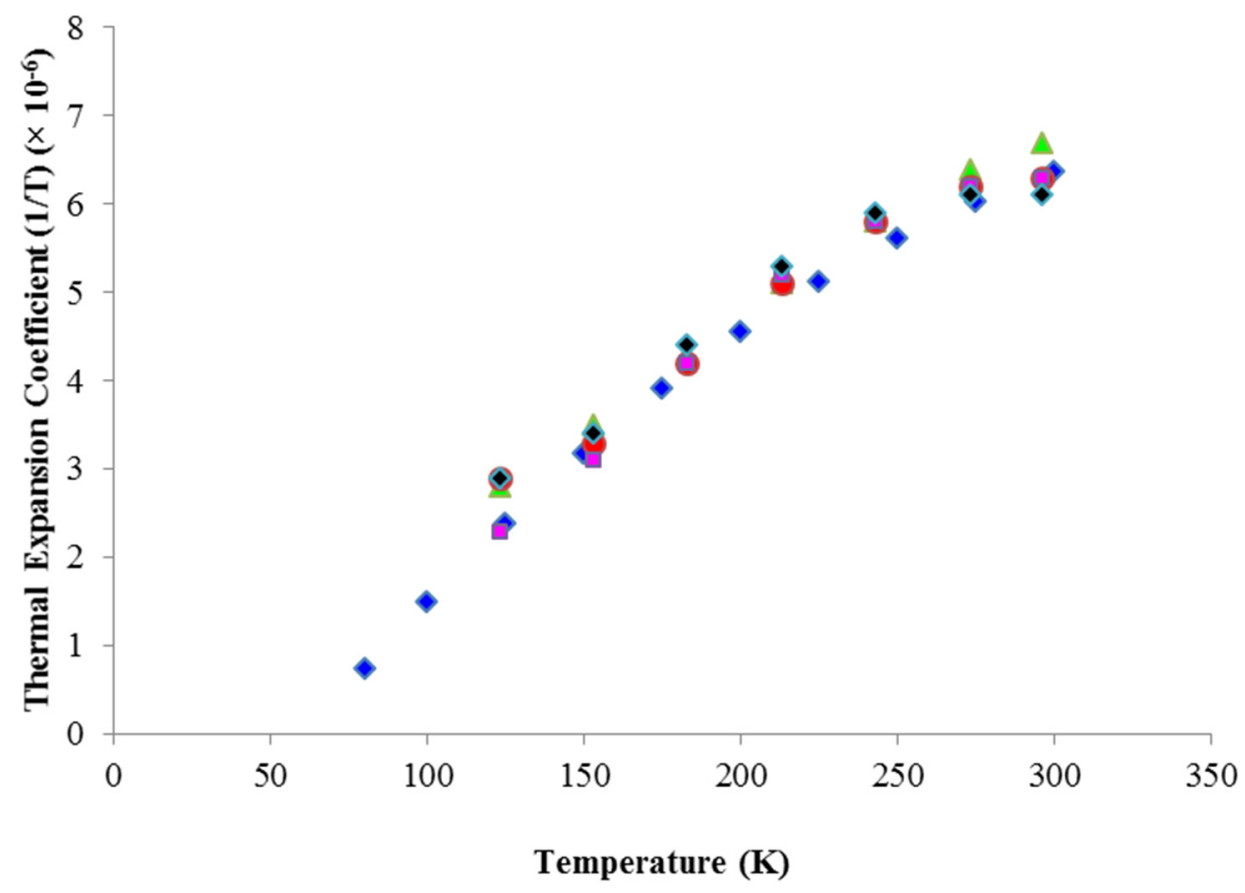

Figure 25. Thermal expansion coefficient as a function of absolute temperature for the undoped isotropic sesquioxide laser materials $\mathrm{Sc}_{2} \mathrm{O}_{3}$ (blue) [30] and $\mathrm{Y}_{2} \mathrm{O}_{3}$ (red) [59], and the 10 at. \% Yb-doped laser material $\mathrm{Yb}: \mathrm{Y}_{2} \mathrm{O}_{3}$ (green) [59], the 1 at. \% $\mathrm{Yb}$-doped material $\mathrm{Yb}: \mathrm{Sc}_{2} \mathrm{O}_{3}$ (pink) [59], and 1 at. \% $\mathrm{Yb}$-doped material $\mathrm{Yb}: \mathrm{Lu}_{2} \mathrm{O}_{3}$ (black) [59]. 


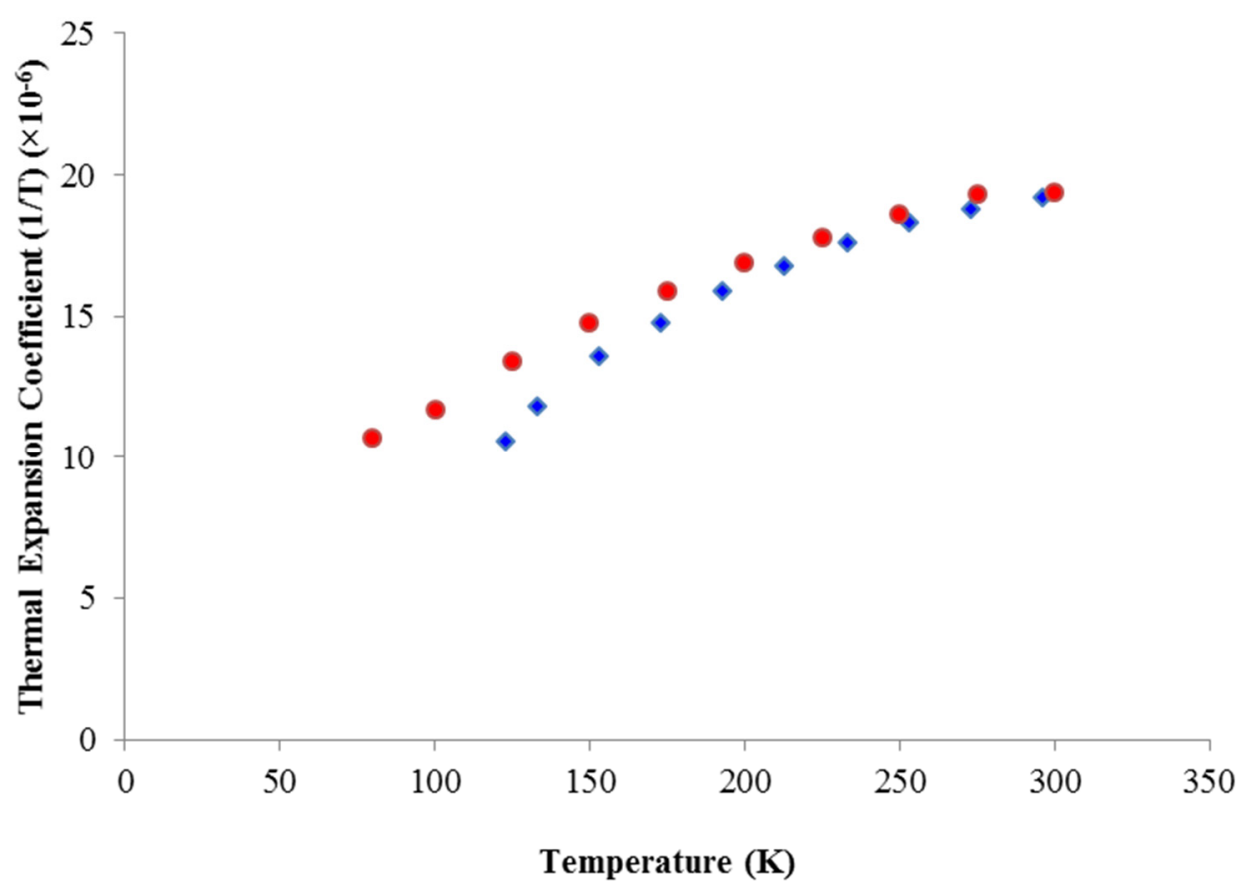

Figure 26. Thermal expansion coefficient as a function of absolute temperature for the isotropic laser material $\mathrm{CaF}_{2}$ (blue) and 10 at. \% Yb-doped $\mathrm{Yb}: \mathrm{CaF}_{2}$ (red) [59].

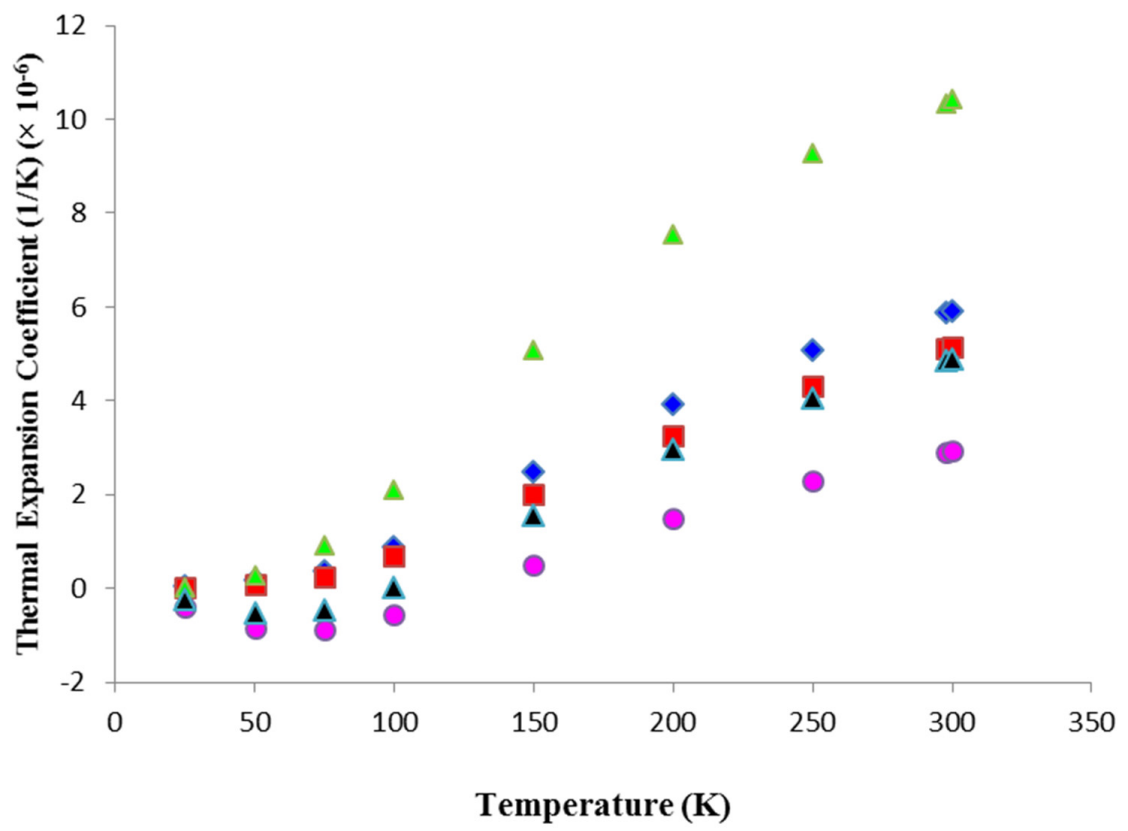

Figure 27. Thermal expansion coefficient as a function of absolute temperature for the uniaxial laser materials $\mathrm{Al}_{2} \mathrm{O}_{3}$ along the $a$ (red) and $c$ axes (blue) [60], $\mathrm{ZnO}$ along the $a$ (black) and $c$ (pink) axes [60], and for the isotropic laser material $\mathrm{MgO}$ (green) [60].

Most of the materials plotted in Figures 20-27 are well behaved in the sense that thermal expansion values are positive and converge towards 0 as temperature is lowered, in agreement with our previous discussion regarding Equation (66). In a few cases, however, the thermal expansion can become negative at lower temperatures, as may be seen in the YALO $a$-axis data in Figure 20, and the $\mathrm{ZnO}$ a and $b$ axes data in Figure 27. In [61] the Grüneisen parameters for YAG and YALO are discussed, and the negative thermal expansion coefficient modeled as an additive negative Grüneisen parameter. 


\subsection{Thermo-Optic Coefficient Measurements}

As with the thermal expansion data of the previous section of this paper, in this section we include only data where nearly complete data sets are available in most of the temperature range of 80-300 K, and ignore single temperature point data. Unlike our previous discussion of the thermal conductivity and thermal expansion coefficient, the theoretical foundation for the thermo-optic coefficient is not nearly as developed, and we refer the reader to previous publications $[2,3,29]$ for more background information. In this section, we show in Figures 28-32 the best data sets we were able to find in the literature, mostly generated at a wavelength of $1064 \mathrm{~nm}$. Other data is available at $632.8 \mathrm{~nm}$ for example, and where $1064 \mathrm{~nm}$ data are unavailable, we will present that data here.

Figure 28 shows thermo-optic coefficient data for the oxide laser materials YAG, LuAG, and YALO, along their principal axes [29]. In Figure 29 we show the very interesting data for the thermo-optic coefficients of the fluoride laser materials YLF and LuLF [29]. The thermo-optic coefficients for both materials are negative across the entire temperature range of interest, for YLF in a monotonically decreasing way as temperature trends towards zero, and in a much more complicated way for LuLF. The negative value of $\mathrm{d} n / \mathrm{d} T$ for YLF is often used to minimize thermal aberrations near room temperature by partially compensating for the positive thermal expansion coefficient. This method is obviously still helpful but of less utility as temperature is lowered for cryogenic laser operation. Figure 30 shows the thermo-optic coefficients for $\mathrm{YVO}_{4}$ and $\mathrm{GdVO}_{4}[3,30]$ along their principal axes, all of which remain positive in the temperature range of interest. The $\mathrm{YSO} b$-axis remains positive as well [30], as shown in Figure 31, but for $\mathrm{CaF}_{2}$ the thermo-optic coefficient is negative for all temperatures, for both doped and undoped cases. The data for $\mathrm{CaF}_{2}$ were obtained for a wavelength of $632.8 \mathrm{~nm}$ [59], but are included here due to the lack of similar data near $1020 \mathrm{~nm}$. For the sesquioxides, data are available only for $\mathrm{Y}_{2} \mathrm{O}_{3}$ and $\mathrm{Sc}_{2} \mathrm{O}_{3}[3,30]$ and shown in Figure 32. While single-point data near $1030 \mathrm{~nm}$ and $300 \mathrm{~K}$ and data collected at $632.8 \mathrm{~nm}$ are available over the temperature range of interest [59], we have not included that data here.

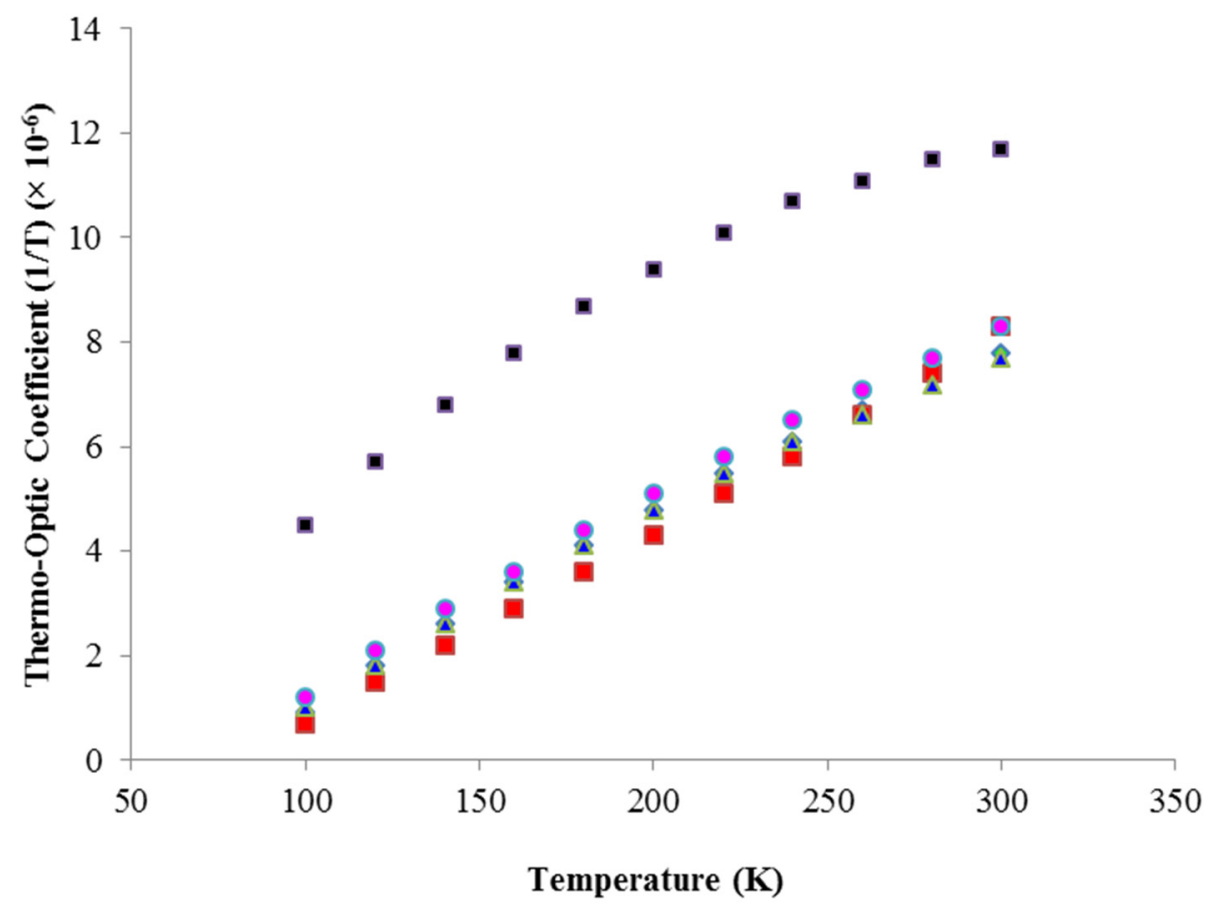

Figure 28. Thermo-optic coefficient as a function of absolute temperature for YAG (green) [29], LuAG (red) [29], and the three principal axes, $a$ (blue), $b$ (black), and $c$ (pink) of YALO [29], measured at the wavelength of $1064 \mathrm{~nm}$. 


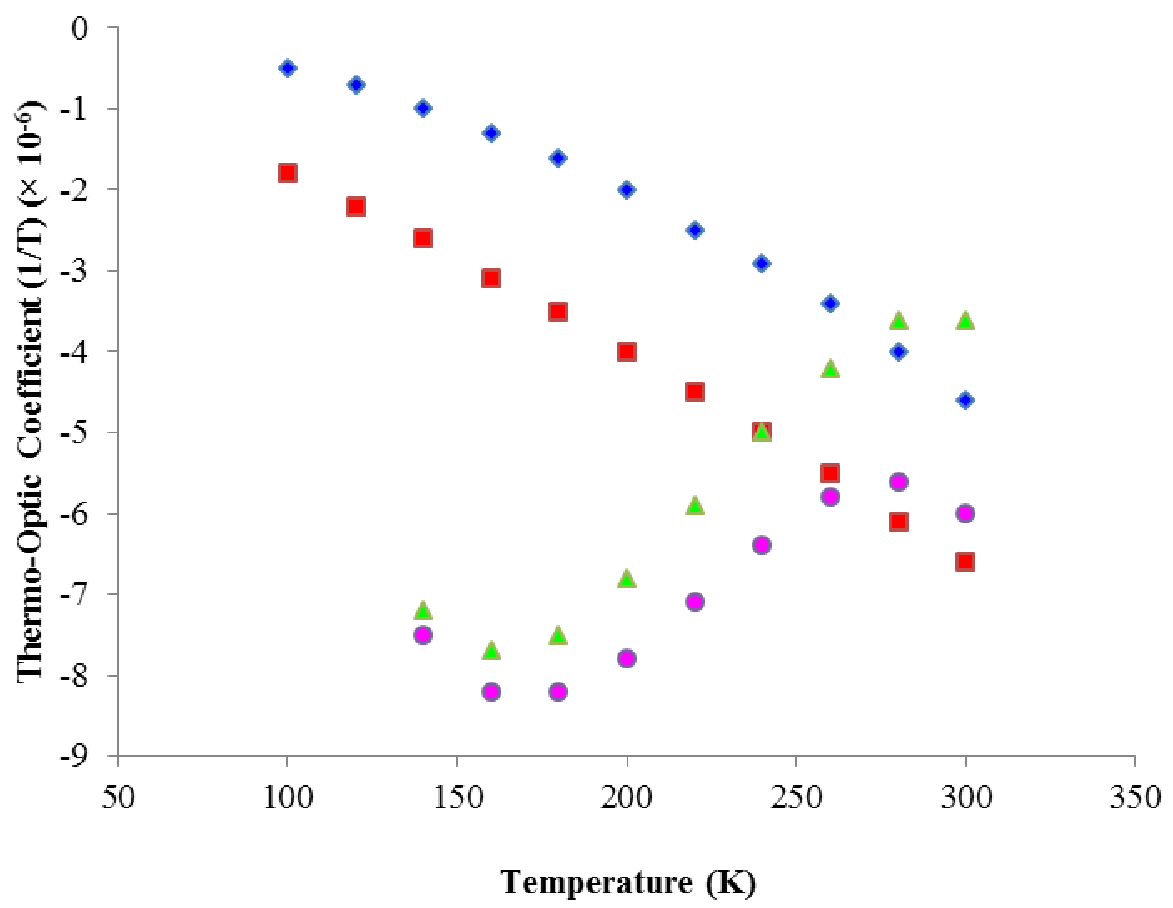

Figure 29. Thermo-optic coefficient as a function of absolute temperature for YLF $a$ (blue) and $c$ (red) axes [29] and LuLF $a$ (green) and $c$ (pink) axes [29], measured at a wavelength of $1064 \mathrm{~nm}$.

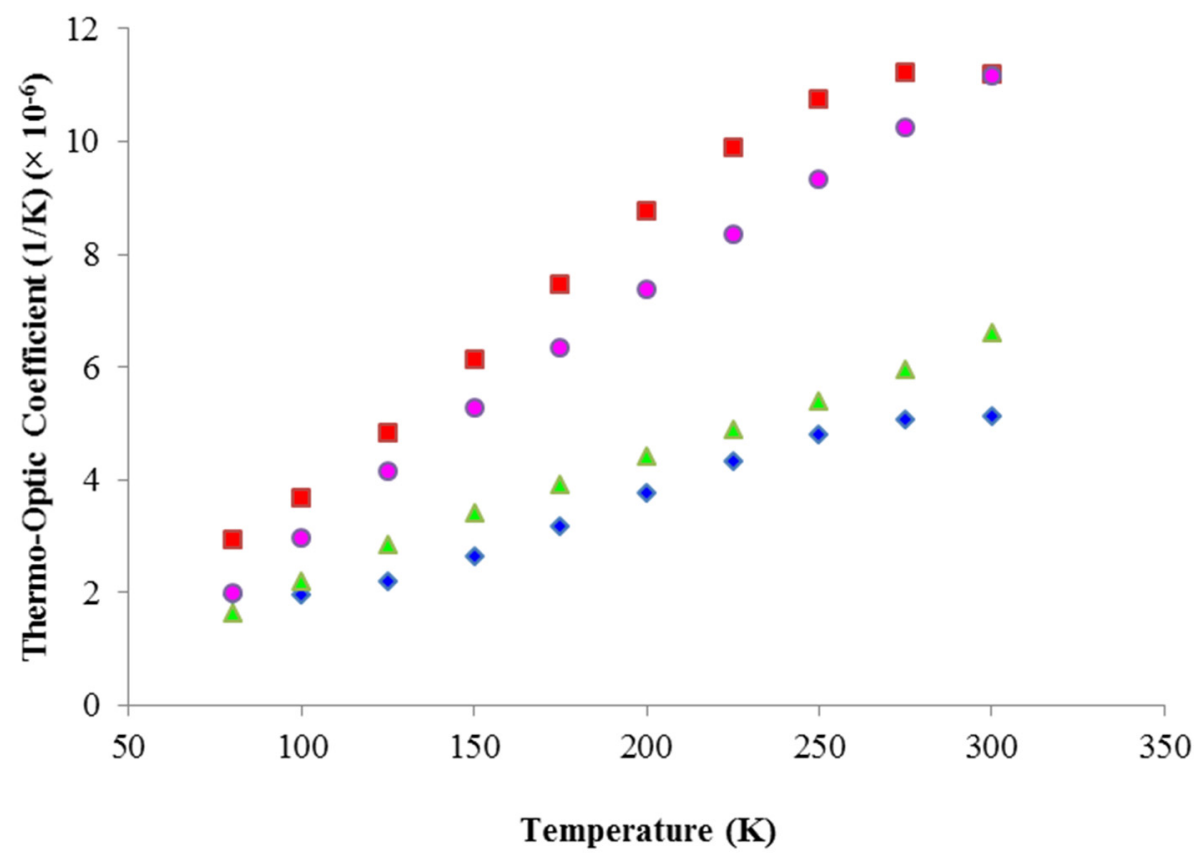

Figure 30. Thermo-optic coefficient as a function of absolute temperature for $\mathrm{YYO}_{4} a$ (red) and $c$ (Blue) axes [30] and $\mathrm{GdVO}_{4} a$ (pink) and $c$ (green) axes [3], measured at a wavelength of $1064 \mathrm{~nm}$. 


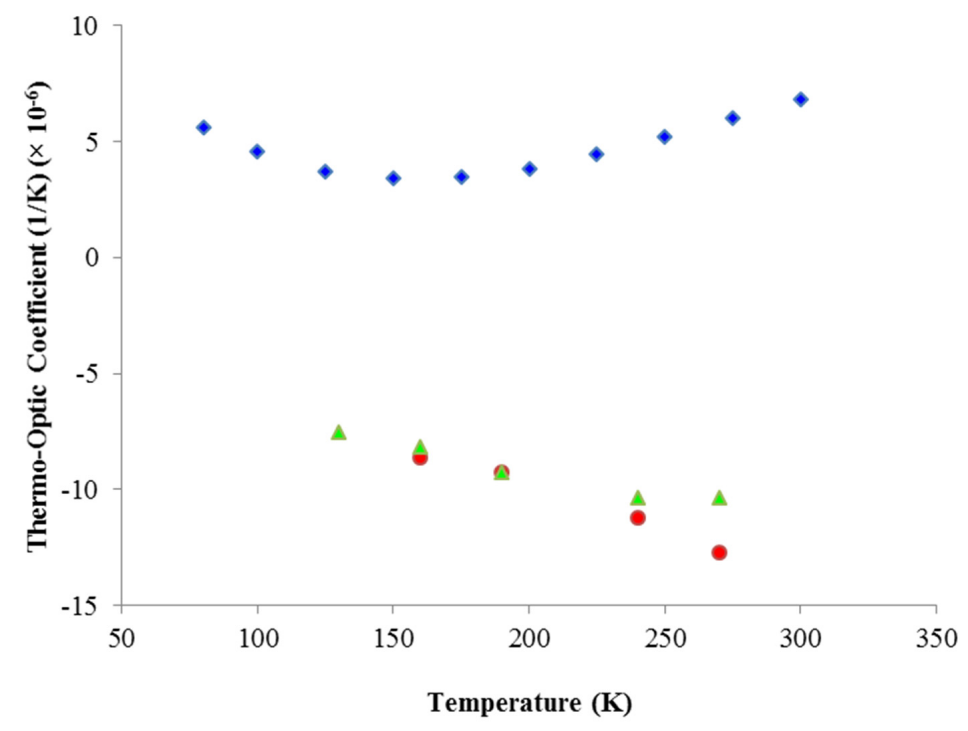

Figure 31. Thermo-optic coefficient as a function of absolute temperature for the YSO $b$-axis (blue) [30], undoped $\mathrm{CaF}_{2}$ (red) [59], and unspecified $\mathrm{Yb}$-doped $\mathrm{CaF}_{2}$ (green) [59]. YSO was measured at a wavelength of $1064 \mathrm{~nm}$ and $\mathrm{CaF}_{2}$ measurements were at $632.5 \mathrm{~nm}$.

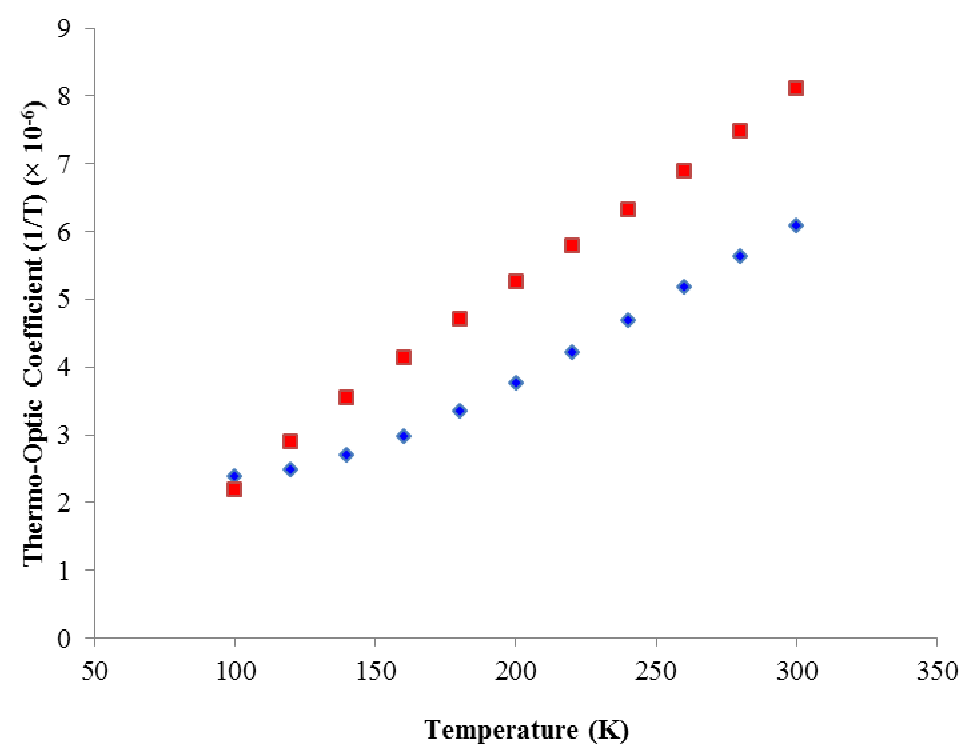

Figure 32. Thermo-optic coefficient as a function of absolute temperature for undoped $\mathrm{Y}_{2} \mathrm{O}_{3}$ (blue) [3] and $\mathrm{Sc}_{2} \mathrm{O}_{3}$ (red) [30], measured at a wavelength of $1064 \mathrm{~nm}$.

\subsection{Elastic Parameters}

The elastic parameters Young's Modulus (E), Poisson's ratio ( $v)$, the bulk modulus $B$ and the related moduli $\mathrm{K}$ and $\mathrm{G}$ have been investigated by a number of researchers. The most comprehensive study was published by Munro [62] who treated single crystal and ceramic materials with varying porosity, and whose data for single crystals we include in Table 2, which lists values for $E_{0}(0 \mathrm{~K}) a$, $E(300 \mathrm{~K}), v$, and $B$. In [62], the Young's modulus values were generated for a temperature of absolute zero, and the values at higher temperatures are determined using the relationship

$$
E(T)=E_{0}(T=0) \cdot(1-a T)
$$


where $\mathrm{E}$ is Young's modulus at temperature $T$ and $a$ is a constant. The bulk moduli values $\mathrm{B}_{0}$ and $\mathrm{B}$ at $300 \mathrm{~K}$ in Table 2 are taken from [62] as well, with $B(\mathrm{~T})$ calculated from the relationship

$$
B(T)=B_{0}(T=0) \cdot(1-b T)
$$

Other values in Table 2 were obtained from [40,63]. The Poisson's ratio values in Table 2 were either calculated at $300 \mathrm{~K}$ using the relationship

$$
v=\frac{1}{2}-\frac{E}{6 B}
$$

or taken from $[40,63]$.

\begin{tabular}{|c|c|c|c|c|c|c|c|}
\hline $\begin{array}{l}\text { Elastic } \\
\text { Parameters }\end{array}$ & $\begin{array}{c}E_{0}(0 \mathbf{K}) \\
(\mathbf{G p a})\end{array}$ & $\begin{array}{c}a \\
\left(10^{-4} / \mathrm{K}\right)\end{array}$ & $\begin{array}{c}E(300 \mathrm{~K}) \\
(\mathrm{Gpa})\end{array}$ & $\begin{array}{c}B_{0}(0 \mathrm{~K}) \\
(\mathrm{Gpa})\end{array}$ & $\boldsymbol{b}\left(\times 10^{-4} / \mathrm{K}\right)$ & $\begin{array}{c}B(300 \mathrm{~K}) \\
(\mathrm{Gpa})\end{array}$ & v \\
\hline $\mathrm{Al}_{2} \mathrm{O}_{3}$ & 393 & 1.33 & 377 & 241 & 0.84 & 235 & 0.23 \\
\hline $\mathrm{CaF}$ & - & - & 110 & - & - & - & 0.30 \\
\hline $\mathrm{Lu}_{2} \mathrm{O}_{3}$ & 204 & 1.03 & 198 & 161 & 0.24 & 160 & 0.29 \\
\hline $\mathrm{MgAl}_{2} \mathrm{O}_{4}$ & 278 & 1.98 & 262 & 187 & 1.97 & 176 & 0.25 \\
\hline $\mathrm{MgO}$ & 310 & 1.63 & 295 & 164 & 1.23 & 158 & 0.19 \\
\hline $\mathrm{Sc}_{2} \mathrm{O}_{3}$ & 229 & 1.22 & 221 & 148 & 0.98 & 144 & 0.24 \\
\hline $\mathrm{Y}_{2} \mathrm{O}_{3}$ & 176 & 1.37 & 169 & 147 & 1.93 & 139 & 0.30 \\
\hline YAG & - & - & 302 & - & - & - & 0.24 \\
\hline $\mathrm{YbAG}$ & - & - & 257 & - & - & - & 0.25 \\
\hline $\mathrm{LiYF}_{4}$ & - & - & 77 & - & - & - & 0.33 \\
\hline
\end{tabular}

Table 2. Young's modulus, bulk modulus, and Poisson's ratio for selected laser materials.

\subsection{Figures of Merit Comparison of Undoped Selected Laser Materials at 300 and $100 \mathrm{~K}$}

Table 3 shows a comparison between the same material at 300 and $100 \mathrm{~K}$, and between different materials, by use of the figures of merit $\Gamma_{T}, \Gamma_{S}$, and $\Gamma$, for laser materials for which most or all of the needed parameters are available in the literature. Most values reported are within a few degrees of 100 or $300 \mathrm{~K}$, and are listed for those values to simplify the presentation. For the heat fraction, here we assume for simplicity that the only contribution to the heat fraction is the quantum defect between the pump and lasing phonons, denoted as $\eta_{h}^{Q D}$. Data used in Table 3 were taken from the references associated to Figures 10-32 as well as Table 2 and its references. It is important to note that the results shown are for undoped materials only.

Concentrating first on the thermal aberrations figure-of-merit $\Gamma_{T}$, we see that for all materials considered, the figure of merit value is significantly larger at $100 \mathrm{~K}$ than at $300 \mathrm{~K}$. This is a consequence of the increased values of $k$ as temperature is lowered, and reduced values of $\alpha$ and $\beta$. The materials with the largest increase in $\Gamma_{T}$ between 300 and $100 \mathrm{~K}$ are YAG, YLF, YALO, $\mathrm{Sc}_{2} \mathrm{O}_{3}, \mathrm{Y}_{2} \mathrm{O}_{3}$, and $\mathrm{Al}_{2} \mathrm{O}_{3}$. These are the materials that will display the largest reductions in thermal aberrations. The material with the smallest increase in the figure-of-merit is $\mathrm{CaF}_{2}$. If this simple comparison, we ignore the fact that additional decreases in thermal aberrations may be achievable by balancing a negative value for $\beta$ with the thermal expansion coefficient to achieve an athermal result as described in connection with Equation (5). YLF and $\mathrm{CaF}_{2}$ for example from benefit from that approach. It should also be appreciated that adding $\mathrm{Yb}$ doping density as an additional parameter, for example, will degrade the expected increases in $\Gamma_{T}$, very significantly in some cases. As we have seen previously in this paper, $\mathrm{Lu}_{2} \mathrm{O}_{3}$ and LuAG have minor decreases in $\mathrm{k}$ as doping density increases, whereas for YAG and $\mathrm{Sc}_{2} \mathrm{O}_{3}$ the drop in thermal conductivity as doping density increases is severe. Perhaps the worst case is $\mathrm{CaF}_{2}$ where for 3 or 5 at. \% Yb doping the thermal conductivity does not increase at any temperature (see Figure 15). 
Table 3. Thermal Conductivity $k$, thermal expansion coefficient $\alpha$, thermo-optic coefficient $\beta$, quantum defect heat fraction $\eta_{h}^{Q D}$, Poisson's ratio $v$, Young's Modulus $E$, and calculated Figures of Merit $\Gamma_{T}, \Gamma_{S}$, and $\Gamma$ for selected laser crystals at 100 and $300 \mathrm{~K}$. Absolute values of negative parameter values were used to calculate figures-of-merit.

\begin{tabular}{|c|c|c|c|c|c|c|c|c|c|c|}
\hline Crystal & $\begin{array}{c}k \\
(\mathrm{~W} /(\mathrm{cm} \cdot \mathrm{K})\end{array}$ & $\begin{array}{c}\alpha \\
\left(10^{-6}\right) \\
(1 / K)\end{array}$ & $\begin{array}{c}\beta \\
\left(10^{-6}\right) \\
(1 / K)\end{array}$ & $\eta_{h}^{Q D}$ & $v$ & $\begin{array}{c}E\left(10^{9}\right) \\
\left(\mathrm{gr} / \mathrm{cm}^{2}\right)\end{array}$ & $\begin{array}{c}\Gamma_{T}\left(10^{10}\right) \\
(\mathrm{W} \cdot \mathrm{K}) / \mathrm{cm}\end{array}$ & $\begin{array}{l}\Gamma_{S}\left(10^{-5}\right) \\
(\mathrm{W} \cdot \mathrm{cm}) / \mathrm{gr}\end{array}$ & $\begin{array}{c}\Gamma \\
(W \cdot \mathrm{cm}) / \mathrm{gr}\end{array}$ & $\begin{array}{c}T \\
\text { (K) }\end{array}$ \\
\hline \multirow{2}{*}{ YAG } & 0.112 & 6.14 & 7.80 & 0.086 & 0.24 & 3.080 & 2.72 & 5.23 & 6.71 & 300 \\
\hline & 0.461 & 1.95 & 0.90 & 0.086 & 0.24 & 3.080 & 305.44 & 67.83 & 753.7 & 100 \\
\hline \multirow{2}{*}{ LuAG } & 0.083 & 6.13 & 8.30 & 0.086 & 0.25 & 2.872 & 1.90 & 4.11 & 4.95 & 300 \\
\hline & 0.254 & 2.46 & 0.70 & 0.086 & 0.25 & 2.872 & 171.5 & 31.35 & 447.86 & 100 \\
\hline \multirow{2}{*}{ YLF-a } & 0.053 & 10.05 & -4.60 & 0.045 & 0.33 & 0.785 & 2.55 & 10.00 & 21.74 & 300 \\
\hline & 0.242 & 3.18 & -0.50 & 0.045 & 0.33 & 0.785 & 338.23 & 144.34 & 453.93 & 100 \\
\hline \multirow{2}{*}{ YLF-c } & 0.072 & 14.31 & -6.60 & 0.045 & 0.33 & 0.785 & 1.69 & 9.54 & 14.45 & 300 \\
\hline & 0.337 & 2.36 & -1.80 & 0.045 & 0.33 & 0.785 & 176.29 & 270.81 & 1504.50 & 100 \\
\hline \multirow{2}{*}{ YALO-a } & 0.117 & 2.32 & 7.70 & 0.096 & 0.23 & 3.220 & 6.82 & 12.56 & 16.31 & 300 \\
\hline & 0.649 & -1.16 & 1.00 & 0.096 & 0.23 & 3.220 & 582.79 & 139.36 & 1393.60 & 100 \\
\hline \multirow{2}{*}{ YALO-b } & 0.100 & 8.08 & 11.70 & 0.096 & 0.23 & 3.220 & 1.10 & 3.08 & 2.63 & 300 \\
\hline & 0.544 & 3.24 & 4.50 & 0.096 & 0.23 & 3.220 & 388.66 & 41.82 & 92.93 & 100 \\
\hline \multirow{2}{*}{ YALO-c } & 0.133 & 8.70 & 8.30 & 0.096 & 0.23 & 3.220 & 1.92 & 3.81 & 4.59 & 300 \\
\hline & 0.776 & 3.00 & 1.20 & 0.096 & 0.23 & 3.220 & 224.54 & 64.43 & 536.92 & 100 \\
\hline \multirow{2}{*}{$\mathrm{Lu}_{2} \mathrm{O}_{3}$} & 0.114 & 6.10 & $7.1^{*}$ & 0.080 & 0.29 & 2.019 & 3.29 & 8.22 & 11.58 & 300 \\
\hline & 0.340 & 2.90 & $2.3 *$ & 0.080 & 0.29 & 2.019 & 63.72 & 51.54 & 224.09 & 100 \\
\hline \multirow{2}{*}{$\mathrm{Sc}_{2} \mathrm{O}_{3}$} & 0.147 & 6.40 & 8.12 & 0.088 & 0.24 & 2.254 & 3.21 & 8.80 & 10.84 & 300 \\
\hline & 0.455 & 0.75 & 2.20 & 0.088 & 0.24 & 2.254 & 313.36 & 265.66 & 1207.55 & 100 \\
\hline \multirow{2}{*}{$\mathrm{Y}_{2} \mathrm{O}_{3}$} & 0.130 & 6.30 & 6.08 & 0.074 & 0.30 & 1.723 & 4.59 & 11.33 & 18.64 & 300 \\
\hline & 0.520 & 0.90 & 2.40 & 0.074 & 0.30 & 1.723 & 325.33 & 156.86 & 653.58 & 100 \\
\hline \multirow{2}{*}{$\mathrm{CaF}_{2}$} & 0.080 & 19.20 & -12.70 & 0.091 & 0.30 & 1.122 & 0.36 & 2.86 & 2.25 & 300 \\
\hline & 0.390 & 10.60 & $-7.5^{*}$ & 0.091 & 0.30 & 1.122 & 5.32 & 25.22 & 23.79 & 100 \\
\hline \multirow{2}{*}{$\mathrm{Al}_{2} \mathrm{O}_{3}-\mathrm{C}$} & 0.330 & 5.15 & 9.80 & 0.335 & 0.23 & 3.844 & 1.95 & 3.83 & 3.91 & 300 \\
\hline & 5.150 & 0.71 & 4.05 & 0.335 & 0.23 & 3.844 & 534.62 & 433.72 & 1070.91 & 100 \\
\hline \multirow{2}{*}{$\mathrm{Al}_{2} \mathrm{O}_{3}$-a } & 0.360 & 5.93 & 12.80 & 0.335 & 0.23 & 3.844 & 1.42 & 3.63 & 2.84 & 300 \\
\hline & 3.440 & 0.90 & 1.90 & 0.335 & 0.23 & 3.844 & 600.51 & 228.55 & 1202.89 & 100 \\
\hline
\end{tabular}

$\left({ }^{*}\right)$-Estimated Values.

For the stress figure-of-merit $\Gamma_{\mathrm{S}}$, the laser material with the largest increase in figure-of-merit between 300 and $100 \mathrm{~K}$ are YAG, YLF, YALO, and $\mathrm{Al}_{2} \mathrm{O}_{3}$. Recall that a large figure-of-merit here means that the stress values achieved are low. Materials with the lowest increase in $\Gamma_{S}$ are $\mathrm{LuAG}$ and $\mathrm{CaF}_{2}$. For the combined figure-of-merit $\Gamma$, the largest value is achieved with YLF, YALO, and $\mathrm{Al}_{2} \mathrm{O}_{3}$. Those are the materials that display the smallest thermal aberrations concurrent with low stress levels.

It is well-known [63], that the thermal rupture modulus $R_{m}$ is defined by the equations

$$
\begin{gathered}
\left(\frac{P_{\mathrm{ex}}^{\mathrm{R}} \gamma}{L}\right)=8 \pi\left(M_{S} \sigma_{\mathrm{f}}\right)=8 \pi \cdot R_{\mathrm{m}}^{\mathrm{R}} \\
\left(\frac{P_{\mathrm{ex}}^{\mathrm{S}} \gamma}{L}\right)=12 \pi\left(M_{S} \sigma_{\mathrm{f}}\right)=12 \pi \cdot R_{\mathrm{m}}^{S}
\end{gathered}
$$

where Equation (72) applies to rod amplifiers and Equation (73) to slab amplifiers. $P_{\mathrm{ex}}^{\mathrm{R}}$ and $P_{\mathrm{ex}}^{\mathrm{S}}$ are the maximum average powers, $L$ the crystal length, $M_{S}$ the material parameter previously defined by Equation (13), $\sigma_{f}$ is the fracture stress, and $R_{\mathrm{m}}^{\mathrm{R}}$ and $R_{\mathrm{m}}^{\mathrm{S}}$ are the rupture moduli for rod and slab amplifiers respectively. The quantity $\gamma$ is the ratio of heat to inversion density. The fracture stress is in most cases the stress achieved at the surface of the amplifier, because surfaces are well-known to have fracture stress values that are in most cases only a fraction of what can be achieved in the bulk. The presence of defects, inhomogeneities, debris, and cracks induced by polishing on surfaces reduce the 
fracture stress; in some cases such cracks and other irregularities can be removed by etching processes. As can be seen from Equations (72) and (73), the maximum extractable average power is achieved when the surface stress is equal to the fracture stress. Because the induced stress in a rod or slab crystal is in all cases inversely proportional to the material parameter $M_{S}$, maximizing it will result in the minimum stresses for any given heat density $Q_{0}$. Because the stress figure-of-merit $\Gamma_{S}$ is $M_{S}$ divided by the constant heat fraction, we see from Equations (72) and (73) that $\Gamma_{S}$ is then also a measure of the average power capability of any laser material. This is simply because maximizing $M_{S}$ results in minimum surface stress, and a small surface stress results in increased average power capability.

In any high average power laser solid-state there is always a tradeoff between wanting to achieve maximum average power whilst at the same time producing the best possible beam quality. These two conflicting requirements can be addressed in a global sense using figures-of-merit $\Gamma_{T}^{w_{1}}$ and $\Gamma_{S}{ }^{w_{2}}$ with weighing factors $\left(0 \leqslant w_{i} \leqslant 1\right)$ that assign no importance $(0)$ or highest importance (1) to the thermal or stress figures-of-merit.

\subsection{Spectroscopic, Lasing, Linear and Nonlinear Optical Parameters}

Ultrafast laser technology has for many decades been dominated by the laser material $\mathrm{Ti}_{\mathrm{Al}} \mathrm{Al}_{2} \mathrm{O}_{3}$, although other wide-bandwidth materials such as laser dyes, Nd:Glass, Yb:Glass, Cr: $\mathrm{BeAl}_{2} \mathrm{O}_{4}$ (Alexandrite), Cr:LiSAF, Cr:LiCAF, and Cr: $\mathrm{Mg}_{2} \mathrm{SiO}_{4}$ (Forsterite) have all played a significant role in the development of ultrafast sources. In the past two decades fiber laser ultrafast sources have come to the fore as well. In this paper, we are interested in exploring the development of ultrafast lasers with both high peak and high average power. The rapid development in the past 15 years of $\mathrm{Yb}$-based ultrafast sources, and the further development of $\mathrm{Ti}^{\mathrm{A}} \mathrm{Al}_{2} \mathrm{O}_{3}$ lasers both seem destined to fill the performance gap between previous ultrafast sources that demonstrated very high peak powers, but in general also very low average powers, and those that are required in the modern era. The almost concurrent development of cryogenic laser technology and the development of new $\mathrm{Yb}$ ultrafast sources heralds the development of a new generation of laser devices that will make possible exciting advancements in a number of scientific fields.

\subsubsection{Spectroscopic and Lasing Parameters}

Cryogenic cooling is not just limited to just mitigating thermal effects in solid-state laser media. Laser kinetics and spectroscopy, as well as many important laser parameters are enhanced as well. Of all the rare-earth and transition-metal lasing ions that have been investigated, $\mathrm{Yb}^{3+}$ is of particular interest. Because of the inefficiency of flashlamp pumping, the development of $\mathrm{Yb}$ lasers was delayed until the introduction of laser diode pump sources. Yb based lasers are free of the efficiency limiting processes of concentration quenching, excited-state absorption, and upconversion that plague many other solid-state lasers. Figure 33 shows a typical $\mathrm{Yb}$ energy level configuration, in this case for the newer laser material $\mathrm{Yb}: \mathrm{Lu}_{2} \mathrm{O}_{3}$. $\mathrm{Yb}$ has only two manifolds, identified as the ground state manifold ${ }^{2} \mathrm{~F}_{7 / 2}$, and an ${ }^{2} \mathrm{~F}_{5 / 2}$ excited state manifold. Most $\mathrm{Yb}$ laser have laser transitions near $1029 \mathrm{~nm}((1,1)$ to $(0,3)$ transition), with some also lasing near $1080 \mathrm{~nm}((1,1)$ to $(0,4))$ transition. The spectral absorption of $\mathrm{Yb}$ baser materials all display bands in the pump regions located near $940 \mathrm{~nm}$, corresponding to the $(0,1)$ to $(1,2)$ transition, and the zero-phonon line absorption near $976 \mathrm{~nm}$ from the $(0,1)$ to $(1,1)$ transition.

Diode-pumping Yb:YAG near $940 \mathrm{~nm}$ can be accomplished using commercially available diode arrays and leads to a small heat fraction of about $9 \%$, whilst pumping near $976 \mathrm{~nm}$ results in a very small heat fraction of $<$ about $5 \%$. Some laser materials like Yb:YAG have such narrow linewidths at $969 \mathrm{~nm}$ that they cannot be efficiently pumped at cryogenic temperatures [3]. The recent development of narrow-band Volume Bragg Grating (VBG) diode pump sources at $976 \mathrm{~nm}$ does however allow the pumping of the zero-phonon line for some laser materials at room temperature and broader band $\mathrm{Yb}$ materials at cryogenic temperatures. It is a significant advantage of $\mathrm{Yb}$ laser materials that they display 
heat fractions that are only a fraction of the more common Nd based materials, primarily because no concentration-quenching occurs, and upconversion is absent.

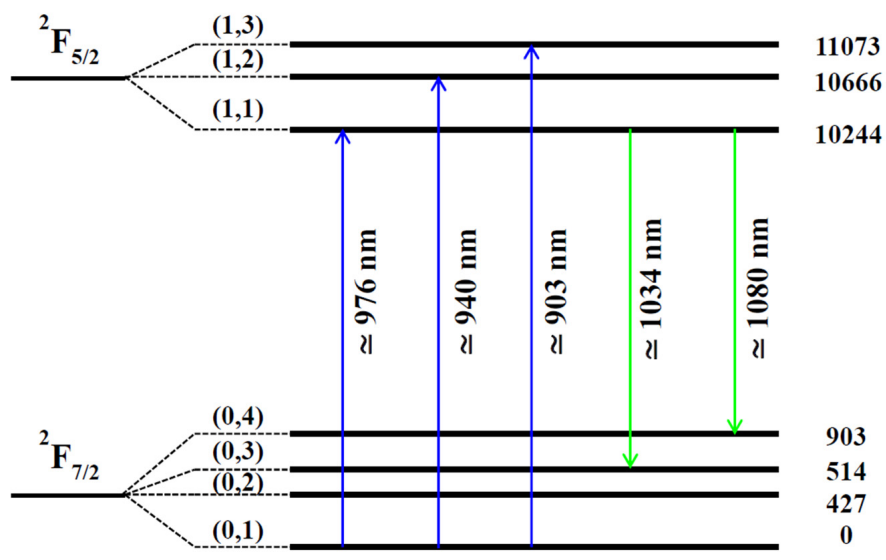

Figure 33. Energy level configuration for $\mathrm{Yb}: \mathrm{Lu}_{2} \mathrm{O}_{3}$.

Another compelling advantage of $\mathrm{Yb}$ based laser materials is that at cryogenic temperatures the finite Boltzmann population of the ground state $(1,3)$ population is effectively eliminated, and thus the need to intensely pump the material at room temperature just to reach transparency is no longer necessary. We can write the pump power density $\rho_{P}$ needed to achieve transparency at $1029 \mathrm{~nm}$ as

$$
\rho_{P}=\frac{n_{d} f_{03} h v_{P}}{\left(f_{03}+f_{11}\right) \cdot \tau_{f}}
$$

In Equation (74), $n_{d}$ is the total $\mathrm{Yb}$ ion density, $v_{\mathrm{P}}$ the frequency of a pump photon, $\tau_{f}$ the upper level fluorescence lifetime, and $f_{03}$ and $f_{11}$ are the Boltzmann occupation factors for the lower laser terminal level, and for the upper laser level respectively (see Figure 33). This equation follows from the transparency condition

$$
f_{11} n_{\mathrm{U}}=f_{03} n_{\mathrm{L}}
$$

where $n_{U}$ and $n_{L}$ are the total population densities in the upper and lower manifolds respectively. Calculation shows that for $\mathrm{Yb}: \mathrm{YAG}$, for example, to pump 1.0 at. \% $\mathrm{Yb}$ to transparency at room temperature requires a pump density of $1.72 \mathrm{~kW} / \mathrm{cm}^{3}$ for $940 \mathrm{~nm}$ pumping. The $940 \mathrm{~nm}$ pump power needed to reach transparency at $77 \mathrm{~K}$, however, is only $0.33 \mathrm{~W} / \mathrm{cm}^{3}$ for 1.0 at. $\% \mathrm{Yb}$ doping.

For $\mathrm{Yb}: \mathrm{YAG}$, the peak stimulated-emission coefficient $\sigma_{\mathrm{e}}$ increases by close to a factor of 5 at $77 \mathrm{~K}$ while the long metastable lifetime of $\sim 951 \mu \mathrm{s}$ at room temperature is maintained at cryogenic temperatures as well. The laser saturation fluence $J_{L}^{S}$ for Yb:YAG can be calculated from the equation

$$
J_{L}^{S}=\frac{h v_{L}}{f_{11} \sigma_{11-03}}
$$

Here, from Figure 33, $\sigma_{11-03}$ is the 11-03 laser transition stimulated-emission spectroscopic cross-section, and $J_{L}^{S}$ is inversely proportional to it. For $\mathrm{CW}$ and pulsed operation, the saturation fluence then decreases by a factor of 5 as well, enhancing the power or energy extraction efficiencies in damage-limited operation. The saturation intensity, found by dividing Equation (76) by the upper level fluorescence lifetime, decreases by the same factor of 5 as well.

Clearly cryogenically cooling $\mathrm{Yb}$ based laser materials at temperatures $<150 \mathrm{~K}$ leads to laser performance benefits that cannot be obtained for room temperature operation. Operating at $300 \mathrm{~K}$ and above, a significant fraction of the pump power is needed to just maintain unity gain, and the smaller stimulated-emission coefficients make damage-limited efficient laser extraction more difficult. CW cryogenic lasers have the potential to provide diffraction-limited output with distortion-free lasing 
elements and with a high wallplug efficiency (laser output average power divided by electrical input power to the diode arrays ,greater than $30 \%$ using a typical demonstrated optical-optical efficiency of $55 \%$ and a typical $55 \%$ efficiency for laser diode sources. As diode laser efficiencies continue to increase with time, perhaps to greater than $80 \%$, and CW optical-optical efficiencies are improved to as high as $85 \%$, $\mathrm{Yb}$ laser wallplug efficiencies may eventually exceed $70 \%$. These efficiency estimates do not of course include any ancillary equipment need to operate the laser.

There are other benefits that accrue from cryogenic cooling. The absorption cross-section for Yb:YAG at the primary pump wavelength of $941 \mathrm{~nm}$ increases by over a factor of two. At $77 \mathrm{~K}$, the doping density can be reduced by about the same factor to achieve the same absorption. Another important feature of the absorption spectrum at $77 \mathrm{~K}$ is that the absorption bandwidth around $941 \mathrm{~nm}$ narrows but still remains very broad. Similar absorption improvements are obtained in other $\mathrm{Yb}$ laser materials as well. This is important because the same diode arrays used to pump at room temperature will work well at $77 \mathrm{~K}$. Lower density, less expensive diode pump sources can also be used at $77 \mathrm{~K}$ for some pumping configurations because a large pump power density is not needed to reach transparency. Another benefit is that the use of end-caps to suppress the strain-distortion at the ends of rod amplifiers can be in most cases be eliminated.

\subsubsection{Linear and Nonlinear Optical Parameters}

The use of cryogenic laser technology to enable the development of advanced laser systems that have both high peak and high average with excellent beam-quality is an approach that has now been proven. So far in this paper we have outlined the many reasons to seriously consider the cryogenic-cooling approach, and have reviewed many of the relevant materials parameters and their dependence on temperature. The path to high-average-power lasers is now well-mapped, however, with regard to ultrafast operation we have just begun to develop the technology because many of the relevant ultrafast parameters for low temperature operation are not known and the database of such properties is at present very limited. The design of ultrafast laser systems requires a detailed knowledge of both the nonlinear optical properties of laser crystals as well as other optical materials used in constructing such systems, and in addition demands a detailed knowledge of the dispersion properties as well. For ultrafast operation, a detailed knowledge of the linear and nonlinear indices of refraction are needed to accomplish competent designs. It is fortunate that for most of the laser materials discussed here, good Sellmeier equations are available in the literature, and are discussed later in this paper, but readers should appreciate that at the present time most reported measurements of the index of refraction and available Sellmeier equations are at or near room temperature. Sellmeier (or Cauchy or Laurent equations) are used to calculate the first, second, third, and fourth-order dispersion parameters needed to achieve dispersion compensation and balancing in ultrafast laser systems, and unfortunately relatively few measurements can be found at cryogenic temperatures. Similarly, the values of the nonlinear index of refraction $n_{2}$ for various crystals and optical materials are well-known only at room temperature.

For ultrafast laser systems with pulsewidths down to the picosecond regime, nonlinear effects such as self-phase-modulation and self-focusing are the principal concerns, and dispersion effects can be mostly ignored. Below about $5-10$ ps, however both nonlinear and dispersion effects must be taken into account to enable good ultrafast designs. Here we first consider just nonlinear effects, and the limitations they place on ultrafast system performance.

\section{Nonlinear Effects in Ultrafast Lasers}

Other than laser-induced optical damage, a subject that we shall discuss in Section 2.7.3, the major performance-limiting factor affecting ultrafast performance in solid-state laser systems is the existence of a finite nonlinear index of refraction $n_{2}$. Much of the early work on inertial-confinement fusion involved the use of flashlamp-pumped laser glasses doped with $\mathrm{Nd}$, driven by picosecond duration Nd:YLF mode-locked oscillators with typical FWHM pulse durations in the range 50-200 ps. 
When scaling such systems to ever larger energy/pulse and peak powers, it was discovered that whole-beam and small-scale self-focusing, caused by the nonlinear index, were the principal limiting factors, and managing and reducing such effects was a major design goal. Good summaries of the understanding and design tools used to design picosecond high-peak-power laser systems may be found in $[37,64]$; in addition a very good summary of the most important papers addressing the phenomena of self-focusing and the design and performance of such systems may be found in [65]. It should be pointed out that in those early systems dispersion management was not a significant design issue and CPA was just in its infancy.

For a laser pule traveling through a nonlinear medium, a finite nonlinear index leads to the temporal phenomena of self-phase-modulation, resulting in a temporally dependent phase modulation. Spatial phase modulation can lead to whole-beam and small-scale self-focusing [37,64]. In general the threshold for the formation of small-scale-self-focusing is significantly lower than that for whole-beam self-focusing. Small-scale self-focusing is caused by either phase or amplitude perturbations of a laser beam, due to dust particles, index inhomogeneities or inclusions. Such perturbations lead to localized self-focusing, resulting ultimately in self-trapped filaments that leave permanent "angel hair" tracks in the laser media. Another source of self-focused filaments is diffraction from hard apertures in the laser system, principally from the edges of laser amplifiers. If uncontrolled, small-scale-self-focusing can lead to a large fraction of the system output beam being lost to large-angle scattering in a halo surrounding the main laser beam. While we will not review in detail the methods for suppressing small-scale-self-focusing in this article, we mention that the imaging of apodized laser beams, the use of vacuum spatial filters, and relay-imaging throughout the entire laser system have been found to be effective tools [37]. Today, the use of the chirped-pulse-amplification (CPA) technique, invented at The Laboratory For Laser Energetics at the University of Rochester by Strickland and Mourou [66], in which ultrafast pulses are spectrally stretched to a much longer pulsewidth for amplification, and then re-compressed at the system output, can be used to largely avoid nonlinear effects as well as take advantage of a higher damage threshold obtained as pulsewidth increases. Calculating in advance the susceptibility of an ultrafast system to nonlinear and damage effects is an important part of ultrafast laser system design, and here we discuss the standard method for doing so.

To manage whole beam and small-scale self-focusing in high peak power laser ultrafast systems, a knowledge of the nonlinear index $n_{2}$ and the corresponding nonlinear coefficient $\gamma_{N}$ is essential. A standard and often-used measure of the severity of nonlinear effects in laser systems is the B-integral [37,64], formally defined as

$$
B(r, z, t)=\Delta \varphi_{N L}(r, z, t)=\frac{2 \pi}{\lambda} \cdot \int \gamma_{N} I(r, z, t) d z
$$

where $B$ is in general a function of the beam radius $r$, propagation location $z$, and time $t, I(r, z, t)$ is the radially, longitudinally, and temporally varying laser intensity, the wavelength $\lambda$, and $z$ is the propagation length parameter. The B-integral is the nonlinear phase accumulation $\Delta \varphi_{N L}(r, z, t)$ due to the nonlinear index $n_{2}$, and in laser systems must be calculated for all optical and gain elements encountered by the propagating beam. It is customary to calculate the maximum value of the B-integral where the intensity $I$ is maximum $(t=0)$. For spatially Gaussian beams, the B-integral value at the beam edge is typically very small, and only the on-axis value for $r=0$ is normally specified. The same holds true for higher-order super-Gaussian beams where the intensity at the beam edge becomes insignificant. For any arbitrary spatial beam profile, is easy to use Equation (72) in a computer code and calculate the B-integral profile at any location in a laser amplifier or system. The nonlinear index can be calculated using the relationship $[37,64]$

$$
n_{2}=\frac{12 \pi^{2}}{n_{0}^{2} c} \cdot \chi^{(3)}
$$


Here, $\chi^{(3)}$ is the third-order susceptibility, and both $n_{2}$ and $\chi^{(3)}$ are expressed in electrostatic units (esu). The relationship between $n_{2}$ and $\gamma_{N}$ is given by the simple formula $[37,67]$

$$
\gamma_{N}=\left(\frac{40 \pi}{c} \cdot 10^{-6}\right) \cdot\left(\frac{n_{2}}{n_{0}}\right)=\left(4.1910^{-3}\right) \cdot\left(\frac{n_{2}}{n_{0}}\right)
$$

and $\gamma_{N}$ is expressed in $\left(\mathrm{cm}^{2} / \mathrm{W}\right)$. We note that in some contributions to the laser literature, $\mathrm{n}_{2}$ is often used to describe $\gamma_{N}$, a confusing practice since they have distinctly different values and units.

Table 3 below shows reported values of $n_{0}, n_{2}$ and $\gamma_{N}$ for a number of optical and laser materials of interest. All reported values are at or near room temperature. In some publications the value of $n_{2}$ was measured, and in others $\gamma_{N}$; Equation (74) was used to provide missing values of either in Table 4.

Table 4. Reported room temperature values of the linear index, nonlinear index and nonlinear coefficient for a number of important laser crystals and optical materials. Also shown are the crystal type (SC-Single Crystal, GM-glassy material, or CER-Ceramic), the E-field orientation (Parallel to specified crystal axis, unspecified, and I-Isotropic), and the measurement wavelength.

\begin{tabular}{|c|c|c|c|c|c|c|c|}
\hline $\begin{array}{c}\text { Crystal } \\
\text { Type }\end{array}$ & Crystal & $\begin{array}{c}\text { E Field } \\
\text { Orientation }\end{array}$ & $\begin{array}{l}\text { Wavelength } \\
\text { (nm) }\end{array}$ & $n_{0}$ & $\begin{array}{c}n_{2}(\text { esu) } \\
10^{-13}\end{array}$ & $\begin{array}{c}\gamma_{\mathrm{N}}\left(\mathrm{cm}^{2} / \mathrm{W}\right) \\
\mathbf{1 0}^{-16}\end{array}$ & Reference \\
\hline SC & $\mathrm{Al}_{2} \mathrm{O}_{3}$ & $\mathrm{E}|| \mathrm{C}$ & 1064 & 1.76 & 1.30 & 3.09 & [68] \\
\hline SC & $\mathrm{Al}_{2} \mathrm{O}_{3}$ & $\mathrm{E}|| \mathrm{C}$ & 532 & 1.78 & 1.40 & 3.30 & {$[68]$} \\
\hline SC & $\mathrm{Al}_{2} \mathrm{O}_{3}$ & $\mathrm{E}|| \mathrm{C}$ & 355 & 1.80 & 1.60 & 3.72 & [68] \\
\hline SC & $\mathrm{Al}_{2} \mathrm{O}_{3}$ & $\mathrm{E} \mid \mathrm{C}$ & 266 & 1.82 & 2.60 & 5.98 & [68] \\
\hline SC & $\mathrm{Al}_{2} \mathrm{O}_{3}$ & $\mathrm{E} \mid \mathrm{C}$ & 650 & 1.77 & 1.36 & 3.21 & [69] \\
\hline SC & $\mathrm{Al}_{2} \mathrm{O}_{3}$ & $\mathrm{E} \mid \mathrm{CC}$ & 700 & 1.77 & 1.33 & 3.17 & [69] \\
\hline SC & $\mathrm{Al}_{2} \mathrm{O}_{3}$ & $\mathrm{E}|| \mathrm{C}$ & 750 & 1.76 & 1.32 & 3.14 & [69] \\
\hline SC & $\mathrm{Al}_{2} \mathrm{O}_{3}$ & $\mathrm{E} \mid \mathrm{CC}$ & 800 & 1.76 & 1.31 & 3.11 & [69] \\
\hline SC & $\mathrm{Al}_{2} \mathrm{O}_{3}$ & $\mathrm{E}|| \mathrm{C}$ & 850 & 1.76 & 1.30 & 3.09 & [69] \\
\hline SC & $\mathrm{Al}_{2} \mathrm{O}_{3}$ & $\mathrm{E} \mid \mathrm{C}$ & 900 & 1.76 & 1.29 & 3.06 & [69] \\
\hline SC & $\mathrm{Al}_{2} \mathrm{O}_{3}$ & $\mathrm{E} \mid \mathrm{C}$ & 950 & 1.76 & 1.28 & 3.04 & [69] \\
\hline SC & $\mathrm{Al}_{2} \mathrm{O}_{3}$ & $\mathrm{E}|| \mathrm{C}$ & 1000 & 1.76 & 1.26 & 3.01 & [69] \\
\hline SC & $\mathrm{Al}_{2} \mathrm{O}_{3}$ & $\mathrm{E}|| \mathrm{C}$ & 1050 & 1.76 & 1.26 & 2.99 & [69] \\
\hline SC & $\mathrm{Al}_{2} \mathrm{O}_{3}$ & EIIC & 1100 & 1.75 & 1.24 & 2.97 & [69] \\
\hline SC & LiSAF & Unspecified & 850 & 1.39 & 1.10 & 3.30 & [70] \\
\hline SC & $\mathrm{LiCAF}$ & Unspecified & 850 & 1.38 & 1.22 & 3.70 & [70] \\
\hline SC & LiSGAF & Unspecified & 850 & 1.39 & 1.10 & 3.30 & [70] \\
\hline $\mathrm{SC}$ & $\mathrm{Mg}_{2} \mathrm{SiO}_{4}$ & Unspecified & 1240 & 1.64 & 0.78 & 2.00 & [70] \\
\hline GM & $\mathrm{SiO}_{2}$ & I & 1064 & 1.45 & 0.95 & 2.74 & [71] \\
\hline GM & $\mathrm{SiO}_{2}$ & I & 527 & 1.46 & 1.05 & 3.00 & [71] \\
\hline GM & $\mathrm{SiO}_{2}$ & I & 351 & 1.48 & 1.27 & 3.60 & [71] \\
\hline GM & BK-7 & I & 1064 & 1.52 & 1.46 & 4.00 & [68] \\
\hline $\mathrm{SC}$ & YAG & I & 1064 & 1.82 & 2.51 & 5.78 & [72] \\
\hline $\mathrm{SC}$ & YAG & I & 1064 & 1.82 & 2.70 & 6.21 & [73] \\
\hline CER & YAG & I & 1064 & 1.82 & 2.49 & 5.73 & [72] \\
\hline SC & YALO & $\mathrm{E} \mid \mathrm{IC}$ & 1064 & 1.91 & 3.33 & 7.30 & [52] \\
\hline SC & YLF & $\begin{array}{l}\text { Unoriented } \\
\text { Measurement }\end{array}$ & 1064 & $\begin{array}{l}1.46(\mathrm{o}) \\
1.48(\mathrm{e})\end{array}$ & 0.60 & 1.72 & [74] \\
\hline SC & LuAG & I & 1064 & 2.14 & 5.50 & 10.77 & [73] \\
\hline $\mathrm{SC}$ & $\mathrm{Y}_{2} \mathrm{O}_{3}$ & I & 1064 & 1.78 & 5.33 & 12.54 & [73] \\
\hline CER & $\mathrm{Y}_{2} \mathrm{O}_{3}$ & I & 1064 & 1.78 & 5.79 & 13.63 & {$[72]$} \\
\hline CER & $\mathrm{Sc}_{2} \mathrm{O}_{3}$ & I & 1064 & 1.85 & 5.32 & 12.05 & [72] \\
\hline CER & $\mathrm{Lu}_{2} \mathrm{O}_{3}$ & I & 1064 & 1.83 & 3.96 & 9.06 & [72] \\
\hline $\mathrm{SC}$ & $\mathrm{MgO}$ & I & 1064 & 1.74 & 1.61 & 3.88 & [73] \\
\hline SC & $\mathrm{MgAl}_{2} \mathrm{O}_{4}$ & I & 1064 & 1.72 & 1.50 & 3.65 & [73] \\
\hline SC & $\mathrm{CaF}_{2}$ & I & 1064 & 1.43 & 0.43 & 1.26 & [73] \\
\hline SC & $\mathrm{YVO}_{4}$ & $\mathrm{E}|| \mathrm{C}$ & 1080 & 2.25 & 8.06 & 15.00 & [75] \\
\hline SC & $\mathrm{YVO}_{4}$ & $\mathrm{E}|| \mathrm{A}$ & 1080 & 1.96 & 8.89 & 19.00 & [75] \\
\hline
\end{tabular}


Table 4. Cont.

\begin{tabular}{|c|c|c|c|c|c|c|c|}
\hline $\begin{array}{l}\text { Crystal } \\
\text { Type }\end{array}$ & Crystal & $\begin{array}{c}\text { E Field } \\
\text { Orientation }\end{array}$ & $\begin{array}{l}\text { Wavelength } \\
\quad(\mathrm{nm})\end{array}$ & $n_{0}$ & $\begin{array}{c}n_{2}(\mathrm{esu}) \\
10^{-13}\end{array}$ & $\begin{array}{c}\gamma_{\mathrm{N}}\left(\mathrm{cm}^{2} / \mathrm{W}\right) \\
\mathbf{1 0}^{-16}\end{array}$ & Reference \\
\hline SC & $\mathrm{YVO}_{4}$ & $\begin{array}{l}\text { Unoriented } \\
\text { Measurement }\end{array}$ & 1064 & $2.06^{(1)}$ & 10.62 & 21.60 & [76] \\
\hline SC & $\mathrm{GdVO}_{4}$ & $\begin{array}{l}\text { Unoriented } \\
\text { Measurement }\end{array}$ & 1064 & $2.08^{(2)}$ & 8.34 & 16.80 & [76] \\
\hline SC & KGW & $\mathrm{E} \mid \mathrm{INm}$ & $800-1600$ & 1.99 & 9.50 & 20.00 & [77] \\
\hline SC & KGW & $\mathrm{E} \mid \mathrm{INp}$ & $800-1600$ & 2.03 & 7.27 & 15.00 & [77] \\
\hline SC & KYW & $\mathrm{E} \mid \mathrm{Nm}$ & 1080 & 2.01 & 4.80 & 10.00 & [75] \\
\hline Gas & Air & I & 400 & 1 & 0.00128 & 0.00536 & [78] \\
\hline Gas & Air & I & 800 & 1 & 0.00072 & 0.00301 & [78] \\
\hline
\end{tabular}

$\mathrm{Al}_{2} \mathrm{O}_{3}$, LiSAF, LiCAF, LiSGAF, $\mathrm{Mg}_{2} \mathrm{SiO}_{4}, \mathrm{SiO}_{2}, \mathrm{YLF}, \mathrm{MgO}, \mathrm{MgAl}_{2} \mathrm{O}_{4}$, and $\mathrm{CaF}_{2}$ all exhibit rather small nonlinear index values in the spectral region 800-1100 nm. YAG, YALO, and LuAG display somewhat larger values, while the sesquioxides $\mathrm{Y}_{2} \mathrm{O}_{3}, \mathrm{Sc}_{2} \mathrm{O}_{3}$, and $\mathrm{Lu}_{2} \mathrm{O}_{3}$ show a substantial increase, followed by the vanadates $\mathrm{YVO}_{4}$ and $\mathrm{GdVO}_{4}$ and the tungstates KGW and KYW which display the largest nonlinear index values. A much larger nonlinear index for many commonly mode-locked materials like $\mathrm{YVO}_{4}, \mathrm{KGW}$, and KYW makes Kerr lens mode locking easier to achieve, but will provide challenges to suppress nonlinear effects in scaled up ultrafast systems consisting of multiple amplifiers. Minimizing B-integral contributions in ultrafast systems utilizing some of these newer materials will likely force designers to consider thin lasing elements such as thin-disk lasers for some applications, however, the thinner the lasing element the more troublesome the suppression of parasitics and amplified-spontaneous-emission, so undoubtedly tradeoffs will need to be made. Also, thin lasing elements have low gain as well, and require multi-passing of the element for good extraction, thus increasing the B-integral and negating any advantage.

The system B-integral, $B_{\text {sys }}$, obtained by adding up the nonlinear phase contributions from all the laser and optical elements encountered by a beam, is a useful measure of the susceptibility of a system to small-scale-self-focusing damage. In general, $B_{\text {sys }}$ values of $<1.5$ radians are very safe, whereas values greater than about 2.5 are very concerning. While these observations are only generally true, minimizing the possibility of system damage due to nonlinear effects is very dependent on system cleanliness and the avoidance of diffraction effects.

In very short-pulse ultrafast $\mathrm{CPA}$ systems such as $\mathrm{Ti}: \mathrm{Al}_{2} \mathrm{O}_{3}$, the generation of pulsewidths $<20-30$ fs requires that the accumulation of second, third, and fourth order dispersion phase in the amplifier portion of the system be compensated, a feat normally accomplished by the use of compressors that can be adjusted to cancel out second, third, and fourth order phase. In femtosecond laser systems the accumulation of nonlinear phase due the B-integral is also an important consideration, and Equation (77) is re-written

$$
B(r, z, t)=\Delta \varphi_{N L}(r, z, t)=2 \pi \cdot \int\left(\frac{1}{\lambda}\right) \gamma_{N} I(r, z, t) d z
$$

reflecting the fact that temporal nonlinear phase Fourier maps into spectral phase. Because of the resulting nonlinear group delay, reducing the B-integral value to $<1$ has advantages in that the compensation of small amounts of nonlinear group delay in the compressor can be achieved.

\section{Dispersion Effects in Ultrafast Lasers}

The understanding of dispersion is a very important aspect of ultrafast laser technology, particularly for pulsewidths <about $10 \mathrm{ps}$, and into the fs and as regimes, and the successful management of dispersion is prerequisite to the success of ultrafast laser systems. Here, we review the description of dispersion and apply the formalism to the calculation of the first, second, third, and 
fourth-order dispersion for a number of important laser materials. We first consider the variation of the linear index of refraction with wavelength or frequency. If we consider an input pulse if field $E_{\mathrm{in}}(t)$ with spectrum $A_{\text {in }}(\omega)$ propagating through a dispersive dielectric crystal, the output pulse can be written as the Fourier relationship

$$
E_{\text {out }}(t)=\left(\frac{1}{2 \pi}\right) \cdot \int A_{\text {in }}(\omega) e^{i \omega t} e^{i \psi(\omega)} d \omega
$$

$\omega$ is the angular frequency, and $\psi(\omega)$ is the spectral phase at frequency $\omega$. Upon passage through a medium of length $\mathrm{L}$, we can relate the spectral phase to the propagation constant of the medium $\beta(\omega)$, through

$$
\psi(\omega)=-\beta(\omega) \cdot L
$$

It is commonplace [70] to expand both $\psi(\omega)$ and $\beta(\omega)$ about a central frequency $\omega_{0}$ using a Taylor series approach, resulting in

$$
\psi(\omega)=\psi\left(\omega_{0}\right)+\frac{\partial \psi}{\partial \omega} \cdot\left(\omega-\omega_{0}\right)+\frac{1}{2} \frac{\partial^{2} \psi}{\partial \omega^{2}} \cdot\left(\omega-\omega_{0}\right)^{2}+\frac{1}{6} \frac{\partial^{3} \psi}{\partial \omega^{3}} \cdot\left(\omega-\omega_{0}\right)^{3}+\ldots
$$

or,

$$
\psi(\omega)=\psi\left(\omega_{0}\right)+\psi_{1} \cdot\left(\omega-\omega_{0}\right)+\frac{1}{2} \psi_{2} \cdot\left(\omega-\omega_{0}\right)^{2}+\frac{1}{6} \psi_{3} \cdot\left(\omega-\omega_{0}\right)^{3}+\ldots
$$

$\beta(\omega)$ is then given by

$$
\beta(\omega)=\beta\left(\omega_{0}\right)+\beta_{1} \cdot\left(\omega-\omega_{0}\right)+\frac{1}{2} \beta_{2} \cdot\left(\omega-\omega_{0}\right)^{2}+\frac{1}{6} \beta_{3} \cdot\left(\omega-\omega_{0}\right)^{3}+\ldots
$$

and we write in general

$$
\psi_{n}=\frac{\partial^{n} \psi}{\partial \omega^{n}}, \beta_{n}=\frac{\partial^{n} \beta}{\partial \omega^{n}}
$$

The derivatives are evaluated at $\omega=\omega_{0}$, and we also have

$$
\psi_{n}=-\beta_{n} \cdot L
$$

$\beta(\omega)$ can also be expressed as

$$
\beta(\omega)=\frac{\omega n(\omega)}{c}
$$

where $c$ is the speed of light. Using Equation (88) we can then calculate the dispersion parameters $\beta_{i}$ as

$$
\begin{aligned}
& \beta_{1}=\frac{d \beta}{d \omega}=n(\omega)+\omega \frac{d n}{d \omega} \\
& \beta_{2}=\frac{d^{2} \beta}{d \omega^{2}}=2 \frac{d n}{d \omega}+\omega \frac{d^{2} n}{d \omega^{2}} \\
& \beta_{3}=\frac{d^{3} \beta}{d \omega^{3}}=3 \frac{d^{2} n}{d \omega^{2}}+\omega \frac{d^{3} n}{d \omega^{3}} \\
& \beta_{3}=\frac{d^{3} \beta}{d \omega^{3}}=4 \frac{d^{3} n}{d \omega^{3}}+\omega \frac{d^{4} n}{d \omega^{4}}
\end{aligned}
$$

Most index of refraction dispersion equations are expressed in terms of wavelength, rather than angular frequency. The most common dispersion equation used is the Sellmeier equation, often written in its' most general form as

$$
n(\lambda)^{2}=A_{0}+\sum_{i} \frac{A_{i} \lambda^{2}}{\left(\lambda^{2}-\lambda_{i}^{2}\right)}
$$


Here $\mathrm{n}$ is the wavelength dependent index of refraction, and $A_{0}, A_{i}$ and $\lambda_{i}$ are fitting constants. In many cases $A_{0}=1$, and $\mathrm{i}$ is usually $\leqslant 4$. Another equation often found in the literature is the Laurent series formula,

$$
n(\lambda)^{2}=A+B \cdot \lambda^{2}+\sum_{i} \frac{C_{i}}{\lambda^{2 i}}
$$

where $A, B$, and $C_{i}$ are constants, and the index $i$ takes the values $1,2,3$, and 4 . The Cauchy equation is also often used, which may be obtained by setting $B=0$ in Equation (94). For the calculations reported below in this paper, the appropriate index equation was converted to angular frequency, resulting in the following frequency dependent Sellmeier equation:

$$
n(\omega)^{2}=A_{0}+\sum_{i} \frac{A_{i} \omega_{i}^{2}}{\left(\omega_{i}^{2}-\omega^{2}\right)}
$$

Table 5 shows calculated values of the index of refraction, the wavelength the values were calculated at, and the first, second, third, and fourth order dispersion coefficients. In addition, references are provided to allow interested readers to know what form of the index equation was used, as well as the values of the constants. We have included crystals and optical materials often used in ultrafast lasers, including Ti: $\mathrm{Al}_{2} \mathrm{O}_{3}, \mathrm{Cr}$ :LiSAF, $\mathrm{Cr}$ :LiCAF, and a number of Yb laser materials. Some entries in Table 5 have also served as a check on the calculational accuracy. For example, for BK-7 at $800 \mathrm{~nm}$ and fused silica $\left(\mathrm{SiO}_{2}\right)$ at $800 \mathrm{~nm}$, the calculated dispersion parameter values agree closely with previously published results [32]. For $\mathrm{Ti}: \mathrm{Al}_{2} \mathrm{O}_{3}$, the dispersion parameters were calculated for a Sellmeier equation used for sapphire grown using the heat exchanger method (HEM) [79], and are similar to previously published results [32], but a one-to-one comparison cannot be made because the data and Sellmeier equations used in [32] were not revealed. The variation of the index of refraction with wavelength can also be slightly different depending upon the crystal growth method, and the corresponding Sellmeier equations will be somewhat different as well, and it is likely that such differences explain the variation between our calculations and [32]. We have included in Table 5 not only legacy materials like LiSAF, LiCAF, YAG, YALO, and YLF, but also the newer sesquioxide laser materials $\mathrm{Y}_{2} \mathrm{O}_{3}, \mathrm{Sc}_{2} \mathrm{O}_{3}$, and $\mathrm{Lu}_{2} \mathrm{O}_{3}$, and the tungstate materials $\mathrm{KGW}, \mathrm{KYW}$, and $\mathrm{KYbW}$. One case deserves mentioning here: that of Cr:LiSAF. We found published values for the second $\left(21.0 \mathrm{fs}^{2} / \mathrm{mm}^{2}\right.$ and third-order (185.0 $\mathrm{fs}^{3} / \mathrm{mm}$ ) dispersion coefficients for the e-axis [70] that do not agree well with our results, nor with those found elsewhere [80]. Values in [70] were calculated using the Sellmeier equations of [81]. We calculated $\beta_{2}$ and $\beta_{3}$ using both the Sellmeier equations of Peterson [81] and Payne [82]. While both Sellmeier equations differ substantially from the measured values, the data of Peterson [81] gave the worst agreement, so in Table 5 we show our calculated values $\left(7.9 \mathrm{fs}^{2} / \mathrm{mm}^{2}\right.$ and $\left(15.7 \mathrm{fs}^{3} / \mathrm{mm}\right)$ obtained using the Payne Sellmeier equation. It has been noted however [80], that even the Payne Sellmeier equations give results considerably different from the measured results. In addition, our results differ from the calculated results of [80], where the reported calculated values using the same Sellmeier equation are $\left(9.7 \mathrm{fs}^{2} / \mathrm{mm}\right)$ and $\left(28.6 \mathrm{fs}^{3} / \mathrm{mm}\right)$. While we have endeavored to calculate accurate values for the dispersion parameters reported in Table 5 , and have checked and compared our results to other values found in the literature where available, readers should be aware that coding long and tedious Sellmeier, Cauchy, Laurent, and other equations and their derivatives up to the fourth order may lead to some inadvertent errors being present.

We conclude this section of the paper by again pointing out that all of the Sellmeier equations and calculated values for the dispersion parameters are for room-temperature operation. It is now well-known that the value of the index of refraction at room temperature are diminished at lower (cryogenic) temperatures by the thermo-optic coefficient $\mathrm{d} n / \mathrm{d} T$. Calculation shows that if the change in index of refraction for YAG for example by integrating the thermo-optic coefficient over the range 300 to $100 \mathrm{~K}$, the index value at $300 \mathrm{~K}$ is affected in the third decimal place. For a variation of the index in the third decimal place, the Sellmeier equation will change as well, and thus will all of the 
dispersion parameters. The same conclusion may be reached for most other crystals as well. It is then important going forward to obtain measurements of the indices of refraction as a function of wavelength at cryogenic temperatures.

Table 5. Calculated room temperature values of the linear index and the first, second, third, and fourth order dispersion parameters for legacy and newer laser crystals of length $1 \mathrm{~mm}$. Also shown are the operating laser wavelengths, as well as references to allow readers to know what Sellmeier or alternative index equation was used in the calculations.

\begin{tabular}{|c|c|c|c|c|c|c|c|}
\hline Crystal & Wavelength (nm) & $n_{0}$ & $\begin{array}{c}\beta_{1} \\
(\mathrm{fs} / \mathrm{mm})\end{array}$ & $\begin{array}{c}\beta_{2} \\
\left(\mathrm{fs}^{2} / \mathrm{mm}\right)\end{array}$ & $\begin{array}{c}\beta_{3} \\
\left.\text { (fs }{ }^{3} / \mathrm{mm}\right)\end{array}$ & $\begin{array}{c}\beta_{4} \\
\left.\text { (fs }{ }^{4} / \mathrm{mm}\right)\end{array}$ & Reference \\
\hline $\begin{array}{c}\mathrm{Al}_{2} \mathrm{O}_{3}-(\mathrm{e}) \\
(\mathrm{HEM})\end{array}$ & 800 & 1.7540 & 5928.1 & 49.9 & 48.3 & -30.0 & [79] \\
\hline $\begin{array}{c}\mathrm{Al}_{2} \mathrm{O}_{3}-(\mathrm{o}) \\
(\mathrm{HEM})\end{array}$ & 800 & 1.7620 & 5960.6 & 42.2 & 48.7 & -36.7 & [79] \\
\hline $\begin{array}{c}\mathrm{Al}_{2} \mathrm{O}_{3}-(\mathrm{e}) \\
(\mathrm{HEM})\end{array}$ & 1030 & 1.7477 & 5912.4 & 11.9 & 81.3 & -127.6 & [79] \\
\hline $\begin{array}{c}\mathrm{Al}_{2} \mathrm{O}_{3}-(\mathrm{o}) \\
(\mathrm{HEM})\end{array}$ & 1030 & 1.7552 & 5946.7 & 7.0 & 87.9 & -146.4 & [79] \\
\hline LiSAF-(e) & 850 & 1.4054 & 4709.2 & 7.9 & 15.7 & -15.0 & [82] \\
\hline LiSAF-(o) & 850 & 1.4074 & 4720.0 & 9.5 & 28.4 & -1.2 & [82] \\
\hline LiCAF-(e) & 760 & 1.3890 & 4654.6 & 23.6 & 13.6 & -3.3 & [83] \\
\hline LiCAF-(o) & 760 & 1.3899 & 4659.5 & 23.1 & 13.7 & -3.9 & [83] \\
\hline YAG & 1060 & 1.8243 & 6148.6 & 60.9 & 68.3 & -46.9 & [84] \\
\hline YAG & 1030 & 1.8153 & 6121.7 & 66.6 & 66.7 & -41.6 & [84] \\
\hline LuAG & 1060 & 1.8279 & 6136.5 & 74.6 & 45.7 & 7.3 & [85] \\
\hline LuAG & 1030 & 1.8249 & 6151.8 & 64.4 & 66.1 & -39.3 & [85] \\
\hline YALO-(a) & 1040 & 1.9341 & 6502.8 & 93.4 & 55.5 & 7.6 & [86] \\
\hline YALO-(b) & 1040 & 1.9258 & 6473.2 & 90.3 & 53.5 & 7.2 & [86] \\
\hline YALO-(c) & 1040 & 1.9140 & 6430.1 & 84.2 & 49.7 & 6.3 & [86] \\
\hline YLF-(e) & 1020 & 1.4705 & 4927.6 & 21.7 & 24.2 & -18.7 & [87] \\
\hline YLF-(o) & 1020 & 1.4483 & 4851.8 & 18.9 & 23.3 & -21.3 & [87] \\
\hline YVO4-(e) & 1060 & 2.1661 & 5947.6 & 341.1 & 305.0 & 69.1 & [88] \\
\hline YVO4-(o) & 1060 & 1.9579 & 6662.4 & 191.7 & 168.5 & 18.2 & [88] \\
\hline KGW-(Np) & 1032 & 1.9829 & 6726.0 & 165.8 & 129.6 & 9.1 & [48] \\
\hline KGW-(Nm) & 1027 & 2.0113 & 6828.7 & 178.7 & 139.5 & 12.6 & [48] \\
\hline KGW-(Ng) & 1024 & 2.0625 & 7005.8 & 216.0 & 141.5 & 3.5 & [48] \\
\hline KYW-(Np) & 1028 & 1.9690 & 6673.0 & 184.4 & 429.5 & 21.3 & [48] \\
\hline KYW-(Nm) & 1028 & 2.0073 & 6814.0 & 207.2 & 495.1 & 26.0 & [48] \\
\hline KYW-(Ng) & 1028 & 2.0514 & 6972.1 & 223.8 & 543.2 & 28.9 & [48] \\
\hline KYbW-(Np) & 1040 & 1.9932 & 6772.2 & 130.5 & 106.2 & -29.9 & [48] \\
\hline KYbW-(Nm) & 1026 & 2.0365 & 6897.3 & 173.2 & 421.5 & 56.3 & [48] \\
\hline KYbW-(Ng) & 1024 & 2.0789 & 7074.3 & 179.6 & 307.7 & 20.9 & [48] \\
\hline $\mathrm{Y}_{2} \mathrm{O}_{3}$ & 1030 & 1.8889 & 6386.6 & 113.9 & 93.2 & -14.0 & [89] \\
\hline $\mathrm{Sc}_{2} \mathrm{O}_{3}$ & 1030 & 1.9654 & 6659.2 & 124.8 & 108.6 & -30.4 & [89] \\
\hline $\mathrm{Lu}_{2} \mathrm{O}_{3}$ & 1030 & 1.9102 & 5691.4 & 126.1 & 100.6 & -15.8 & [89] \\
\hline $\mathrm{CaF}_{2}$ & 1030 & 1.4287 & 4784.1 & 18.4 & 20.2 & -14.7 & [52] \\
\hline BK-7, N-BK-7 & 800 & 1.5108 & 5088.8 & 44.6 & 32.0 & -10.0 & [52] \\
\hline BK-7, N-BK-7 & 1030 & 1.5071 & 5070.2 & 25.1 & 44.7 & -49.8 & [52] \\
\hline SF10 & 800 & 1.7112 & 5836.7 & 159.2 & 102.9 & 33.4 & [90] \\
\hline SF10 & 1030 & 1.7030 & 5766.4 & 108.1 & 97.3 & -15.7 & [90] \\
\hline SF11 & 800 & 1.7648 & 6036.4 & 189.4 & 124.1 & 48.3 & [52] \\
\hline SF11 & 1030 & 1.7553 & 5952.9 & 128.9 & 113.0 & -7.3 & [52] \\
\hline N-SF14 & 800 & 1.7429 & 5957.1 & 176.4 & 117.6 & 41.6 & [52] \\
\hline N-SF14 & 1030 & 1.7337 & 5879.7 & 118.3 & 110.2 & -17.7 & [52] \\
\hline Crystal $\mathrm{SiO}_{2}(\mathrm{e})$ & 1030 & 1.5282 & 5189.3 & 0.4 & 98.8 & -193.5 & [91] \\
\hline
\end{tabular}


Table 5. Cont.

\begin{tabular}{|c|c|c|c|c|c|c|c|}
\hline Crystal & Wavelength (nm) & $n_{0}$ & $\begin{array}{c}\beta_{1} \\
(\mathrm{fs} / \mathrm{mm})\end{array}$ & $\begin{array}{c}\beta_{2} \\
\left(\mathrm{fs}^{2} / \mathrm{mm}\right)\end{array}$ & $\begin{array}{c}\beta_{3} \\
\left(\mathrm{fs}^{3} / \mathrm{mm}\right)\end{array}$ & $\begin{array}{c}\beta_{4} \\
\left(\mathrm{fs}^{4} / \mathrm{mm}\right)\end{array}$ & Reference \\
\hline Crystal $\mathrm{SiO}_{2}$ (o) & 1030 & 1.5342 & 5162.8 & 25.8 & 46.2 & -53.6 & [91] \\
\hline Crystal $\mathrm{SiO}_{2}$ (e) & 800 & 1.5348 & 5200.6 & 38.1 & 50.4 & -43.9 & [91] \\
\hline Crystal $\mathrm{SiO}_{2}$ (o) & 800 & 1.5381 & 5181.9 & 45.8 & 32.1 & -12.0 & [91] \\
\hline $\begin{array}{l}\text { Fused Silica } \\
\text { (SiO2) }\end{array}$ & 800 & 1.4533 & 4890.5 & 36.1 & 27.3 & -11.0 & [92] \\
\hline $\begin{array}{l}\text { Fused Silica } \\
\qquad\left(\mathrm{SiO}_{2}\right)\end{array}$ & 1030 & 1.4500 & 4875.7 & 19.0 & 40.4 & -49.8 & [92] \\
\hline
\end{tabular}

\subsubsection{Optically-Induced Laser Damage}

Perhaps the most important parameter in the design of any laser system is the laser-induced damage threshold (LIDT), the value of fluence, intensity, or average power that if exceeded, will result in irreversible damage to at least one (usually critical) optical or lasing element in the laser system. If the weakest component in a laser system has an LIDT that is a fraction of the laser saturation fluence, one cannot obtain a high overall system efficiency because for high extraction efficiency the input laser fluence must be at least equal to the saturation fluence, and in the case of Gaussian beams, many times the saturation fluence [28]. A small saturation fluence is obtained for laser materials with a large stimulated-emission cross-section (see Equation (76)). Because the LIDT has a laser pulsewidth dependence that results in picosecond pulses having a smaller damage threshold than nanosecond pulses, and femtosecond less than picosecond [93], it was a challenge in early ultrafast lasers to be able to scale laser output due to the limiting value of LIDT's in the picosecond and particularly in the femtosecond regime. It was because of those damage limitations, as well as to avoid the type of nonlinear effects discussed in the aforementioned, that the technique of CPA was invented [66]. Using $\mathrm{CPA}$, a short femtosecond pulse for example is stretched using diffraction gratings or other means, to the picosecond regime where damage thresholds are substantially larger, After stretching, the initial pulse is amplified in a following beam line of amplifiers where the larger pulsewidth allows nonlinear effects to be avoided or minimized, and where more efficient extraction can be obtained due to the larger damage threshold. After amplification, the stretched pulse is compressed, in many cases using a diffraction grating compressor, to restore the pulsewidth close to its' initial value. Managing the accumulated dispersion, and compensating for it using certain types of compressor configurations determines how close to the initial pulse width the final compressed pulse comes.

The literature of LIDT's is vast. Interested readers that have a need to thoroughly explore the field may consult the book by Wood [94], as well as the many publications of the Annual Boulder Damage Symposium (Boulder, CO, USA), a conference that celebrated its 45th year in 2013. The LIDT of laser and optical materials is a consequence of the many physical mechanisms connected with the high fields achievable with laser. When intense beams are transmitted through crystals or glasses, many nonlinear effects such as harmonic generation, self-focusing and self-phase modulation, nonlinear absorption, excited-state absorption, stimulated Brillouin or Raman scattering, and others may occur, and contribute to irreversible damage. In addition electron avalanches may occur in the laser in response to a strong E field, and plasmas may form on a surface or internally. High average power effects, that are now becoming more and more important, can be caused by absorption and melting of dielectric crystals or dielectric coatings, and can lead to distortion depolarization, and ultimately fracture of crystals or other optics. It is a general observation, supported by measurements, that the LIDT of surfaces are substantially smaller than bulk LIDT's. LIDT's are a strong function of the laser wavelength, with visible and UV LIDT's much less than those obtained around $1000 \mathrm{~nm}[93,94]$. The pulsewidth dependence of LIDT's is rather simple in the nanosecond regime, varying as the pulsewidth to the $1 / 2$ power $[93,94]$, however for picosecond and femtosecond pulses the dependence is more complicated [95]. 
It is a shortcoming of the present state-of-the-art that high average power effects have received far less attention than high peak power effects. Now that we are witnessing the advent of laser systems that have concurrent high and peak average powers, much attention will be focused in the near future on determining the LIDT's of laser crystals and optics under very extreme conditions. LIDT's at low temperature are scarce and in most cases have not been measured. Recently a publication reported a reduced damage threshold at cryogenic temperatures relative to the room temperature value [96]. This in contrast to a number of publications that have reported increased damage thresholds at cryogenic temperatures [97-99]. Increased laser damage threshold measurement efforts at cryogenic temperatures are needed to further our understanding of the design of cryogenic laser devices and to increase our knowledge of the scaling of the damage threshold with temperature.

\subsubsection{Spectral and Laser Properties of Traditional and New Laser Materials}

The design and performance of ultrafast lasers is critically dependent upon the spectral and laser properties of the amplifier materials used. We have already, in the aforementioned, discussed the important nonlinear parameters and the dispersion parameters needed to optimize the performance of ultrafast lasers. Here, we review the spectral properties of a number of important laser materials, including a number of legacy ultrafast materials as well as some newer laser materials, all based upon the $\mathrm{Yb}^{3+}$ lasing ion. A more complete compilation of properties for most $\mathrm{Yb}$ based laser materials will be presented elsewhere [100]; here we show only $\mathrm{Yb}$ materials for which both room and cryogenic temperature data are available. Table 6 shows the laser material, the crystal classification (anaxial (A) and uniaxial (U)), the absorption span $\delta \lambda_{a b s}$ (FWHM) and wavelength of peak absorption $\lambda_{a b s}^{P}$, the absorption bandwidth $\Delta \lambda_{\text {abs }}$ (FWHM), peak absorption cross-section $\sigma_{a b s}^{P}$, the wavelength of the peak stimulated-emission cross-section $\lambda_{e m}^{P}$, the emission bandwidth $\Delta \lambda_{e m}$ (FWHM), the value of the peak stimulated-emission cross-section $\sigma_{e m}^{P}$, the upper level $\left({ }^{2} \mathrm{~F}_{5 / 2}\right)$ fluorescence lifetime $\tau_{F}$, the absolute temperature, and the references from whence the data were taken $[3,6,30,101-110]$.

The three legacy laser materials shown, $\mathrm{Ti}: \mathrm{Al}_{2} \mathrm{O}_{3}, \mathrm{Cr}$ :LiSAF, and $\mathrm{Cr}$ :LiCAF, are uniaxial and we show data for the cases $\mathrm{E} I \mathrm{I} \mathrm{c}$ and $\mathrm{E} I \mathrm{I}$ a, and at room temperature. While Ti: $\mathrm{Al}_{2} \mathrm{O}_{3}$ is often cooled to near $77 \mathrm{~K}$ to reduce thermal aberrations [31,33-35], little data is available in the literature regarding the spectral properties at that temperature. For Cr:LiSAF and Cr:LiCAF, we were not able to find any cryogenic data available in the literature. The bandwidths for these materials indicate that for transform-limited Gaussian pulses, pulsewidths of as short as 4, 6, and $7 \mathrm{fs}$ can be achieved, in the absence of gain-narrowing.

For the $\mathrm{Yb}$ materials $\mathrm{Yb}: \mathrm{CaF}_{2}, \mathrm{Yb}: \mathrm{LuAG}, \mathrm{Yb}: \mathrm{YAG}$, and $\mathrm{Yb}: \mathrm{YLF}$, complete sets of spectral parameters are available at room temperature and near $77 \mathrm{~K}$, and both are shown in Table 6 . We have also included the recently developed sesquioxide laser materials $\mathrm{Yb}: \mathrm{Lu}_{2} \mathrm{O}_{3}, \mathrm{Yb}: \mathrm{Sc}_{2} \mathrm{O}_{3}$, and $\mathrm{Yb}: \mathrm{Y}_{2} \mathrm{O}_{3}$, but complete data sets are available at present only at $300 \mathrm{~K}$. The first $\mathrm{Yb}$ laser material to be investigated at cryogenic temperature was Yb:YAG [1], but the emission bandwidth is narrow and only capable of producing Gaussian transform-limited pulses of about $1 \mathrm{ps}$ at $80 \mathrm{~K}$, due to the narrower gain bandwidth [3]. The material Yb:LuAG, very similar to $\mathrm{Yb}: \mathrm{YAG}$, is capable of producing $1.4 \mathrm{ps}$ pulses. The first Yb:YLF laser operated at cryogenic temperatures was reported in [109]. Yb:YLF is an interesting ultrafast cryogenic laser material, due to the wide emission bandwidths for both $\pi$ and $\sigma$ polarizations. For E I I c and E I I a at $300 \mathrm{~K}$, the shortest transform-limited Gaussian pulsewidths that can be obtained are about 43 and $57 \mathrm{fs}$ at $300 \mathrm{~K}$, and 191 and $55 \mathrm{fs}$ at $79 \mathrm{~K}$. The material is robust and has relatively high thermal and stress figures of merit (Table 3). The first demonstration of combining two $\mathrm{Yb}$ laser materials together (Yb:YAG and $\mathrm{Yb}: \mathrm{GSAG}$ ) to broaden the gain bandwidth, produced recompressed shorter pulses of $\sim 1.6$ ps duration [110]. Not all of the spectral properties of Yb:GSAG are known, particularly at room temperature, but we have included the known properties in Table 6 . 
Table 6. Spectral properties of legacy and Yb-based ultrafast laser materials at room and cryogenic temperatures.

\begin{tabular}{|c|c|c|c|c|c|c|c|c|c|c|}
\hline Crystal & Crystal Type & $\begin{array}{c}\delta \lambda_{a b s}, \lambda_{a b s}^{P} \\
(\mathrm{FWHM})(\mathrm{nm})\end{array}$ & $\begin{array}{c}\Delta \lambda_{a b s}(\mathrm{FWHM}) \\
(\mathrm{nm})\end{array}$ & $\sigma_{a b s}^{P}\left(\mathrm{~cm}^{2}\right)\left(10^{-20}\right)$ & $\lambda_{e m}^{P}(\mathrm{~nm})$ & $\begin{array}{c}\Delta \lambda_{e m}(\mathrm{FWHM}) \\
(\mathrm{nm})\end{array}$ & $\begin{array}{c}\sigma_{e m}^{P}\left(\mathrm{~cm}^{2}\right) \\
\left(10^{-20}\right)\end{array}$ & $\tau_{F}(\mu \mathrm{s})$ & Temp (K) & Refs \\
\hline \multirow{2}{*}{$\begin{array}{c}\text { Ti3+:Al2O3 } \\
\text { El Ic }(\pi)\end{array}$} & \multirow[b]{2}{*}{$\mathrm{U}$} & $455-580$ & \multirow[b]{2}{*}{120.0} & \multirow[b]{2}{*}{6.45} & \multirow[b]{2}{*}{790} & \multirow[b]{2}{*}{225.0} & \multirow[b]{2}{*}{3.0} & \multirow[b]{2}{*}{3.2} & \multirow[b]{2}{*}{300} & \multirow[b]{2}{*}[6,101]{} \\
\hline & & 493 & & & & & & & & \\
\hline Ti3+:Al2O3 & \multirow[b]{2}{*}{$\mathrm{U}$} & $455-575$ & \multirow[b]{2}{*}{122.0} & \multirow[b]{2}{*}{2.79} & \multirow[b]{2}{*}{800} & \multirow[b]{2}{*}{236.0} & \multirow[b]{2}{*}{1.5} & \multirow[b]{2}{*}{3.2} & \multirow[b]{2}{*}{300} & \multirow[b]{2}{*}[6,101]{} \\
\hline $\mathrm{E} \mid \mathrm{Ia}(\sigma)$ & & $\begin{array}{c}493 \\
\text { Peak }\end{array}$ & & & & & & & & \\
\hline Cr3+:LiSAF & \multirow[b]{2}{*}{$\mathrm{U}$} & $600-703$ & \multirow[b]{2}{*}{97.7} & \multirow[b]{2}{*}{5.4} & \multirow[b]{2}{*}{850} & \multirow[b]{2}{*}{192.0} & \multirow[b]{2}{*}{4.8} & \multirow[b]{2}{*}{67} & \multirow[b]{2}{*}{300} & \multirow[b]{2}{*}{ [102] } \\
\hline ElIc & & $\begin{array}{l}637 \\
\text { Peak }\end{array}$ & & & & & & & & \\
\hline Cr3+:LiSAF & \multirow[b]{2}{*}{$\mathrm{U}$} & $593-693$ & \multirow[b]{2}{*}{102.3} & \multirow[b]{2}{*}{2.6} & \multirow[b]{2}{*}{850} & \multirow[b]{2}{*}{171.4} & & & & \\
\hline Ella & & $\begin{array}{c}637 \\
\text { Peak }\end{array}$ & & & & & 1.6 & 67 & 300 & [102] \\
\hline Cr3+:LiCAF & & $585-680$ & & & & & & & & \\
\hline ElIc & $\mathrm{U}$ & $\begin{array}{l}647 \\
\text { Peak }\end{array}$ & 85.8 & 2.95 & 763 & 127.0 & 1.3 & 175 & 300 & [102] \\
\hline $\mathrm{Cr} 3+: \mathrm{LiCAF}$ & & $580-680$ & & & & & & & & \\
\hline $\mathrm{Yb} 3+: \mathrm{CaF} 2$ & A & $\begin{array}{c}915-970 \\
979\end{array}$ & $\begin{array}{l}53.3 \\
21.8\end{array}$ & $\begin{array}{l}0.83 \\
0.57\end{array}$ & 1030 & 26.7 & 0.25 & 1900 & 300 & [103] \\
\hline $\mathrm{Yb} 3+: \mathrm{CaF} 2$ & A & $\begin{array}{c}915-970 \\
980\end{array}$ & $\begin{array}{l}53.3 \\
2.93\end{array}$ & $\begin{array}{l}0.88 \\
1.57\end{array}$ & $\begin{array}{c}1033 \\
990\end{array}$ & $\begin{array}{l}43.3 \\
10.7\end{array}$ & $\begin{array}{l}0.50 \\
0.83\end{array}$ & 1900 & 77 & [103] \\
\hline $\mathrm{Yb} 3+: \mathrm{Lu} 2 \mathrm{O} 3$ & A & $\begin{array}{c}930-953 \\
978\end{array}$ & $\begin{array}{c}11.04 \\
3.49\end{array}$ & $\begin{array}{l}0.80 \\
3.81\end{array}$ & $\begin{array}{l}1033 \\
1078\end{array}$ & $\begin{array}{l}13.2 \\
14.3\end{array}$ & $\begin{array}{l}1.89 \\
0.89\end{array}$ & 820 & 300 & $\begin{array}{l}{[104]} \\
{[105]}\end{array}$ \\
\hline Yb3+:LuAG & A & $\begin{array}{c}915-945 \\
970\end{array}$ & $\begin{array}{l}30.0 \\
2.75\end{array}$ & $\begin{array}{l}0.94 \\
1.01 \\
\end{array}$ & 1030 & 3.4 & 2.50 & 950 & 295 & $\begin{array}{l}{[104]} \\
{[106]} \\
\end{array}$ \\
\hline Yb3+:LuAG & $\mathrm{A}$ & $\begin{array}{c}915-945 \\
970\end{array}$ & $\begin{array}{l}27.0 \\
1.10\end{array}$ & $\begin{array}{l}1.73 \\
2.47\end{array}$ & 1030 & 1.1 & 13.90 & 950 & 100 & $\begin{array}{l}{[104]} \\
{[106]}\end{array}$ \\
\hline $\mathrm{Yb3+:Sc2O3}$ & A & $\begin{array}{l}942 \\
975\end{array}$ & $\begin{array}{l}26.2 \\
2.4\end{array}$ & $\begin{array}{l}0.87 \\
4.40\end{array}$ & $\begin{array}{l}1041 \\
1095\end{array}$ & $\begin{array}{l}13.4 \\
10.7\end{array}$ & $\begin{array}{l}1.44 \\
0.33\end{array}$ & 800 & 300 & [107] \\
\hline $\mathrm{Yb3+:Y2O3}$ & A & $\begin{array}{l}950 \\
977\end{array}$ & $\begin{array}{l}5.96 \\
3.97\end{array}$ & $\begin{array}{l}0.87 \\
2.40\end{array}$ & $\begin{array}{l}1031 \\
1076\end{array}$ & $\begin{array}{l}14.3 \\
15.6\end{array}$ & $\begin{array}{l}1.06 \\
0.42\end{array}$ & 850 & 300 & [107] \\
\hline
\end{tabular}


Table 6. Cont

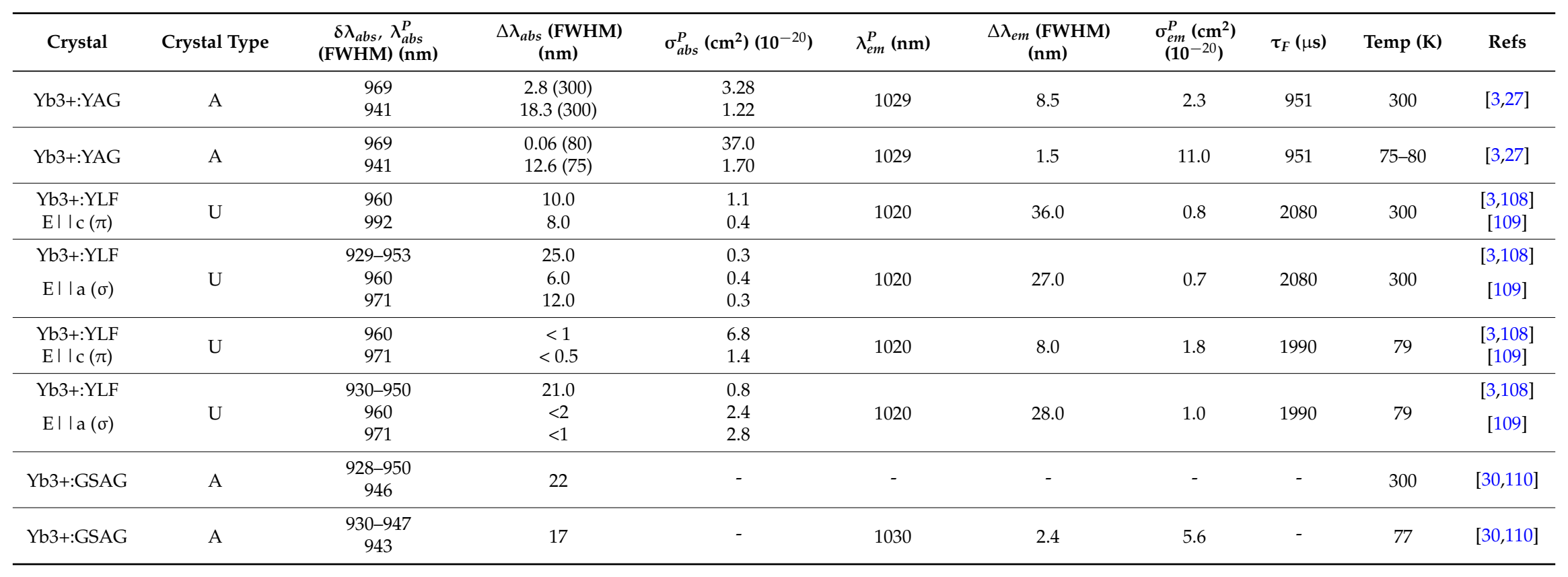


$\mathrm{Yb}: \mathrm{CaF}_{2}$ has come to the fore recently because of its broad emission bandwidth, resulting in $<100 \mathrm{fs}$ pulsewidths from a mode-locked laser recently [111]. From Table 6, we see that it has a wide emission bandwidth, particularly at $77 \mathrm{~K}$. The Gaussian transform-limited pulsewidths expected are $59 \mathrm{fs}$ and $37 \mathrm{fs}$ at 300 and $77 \mathrm{~K}$ respectively. As we have seen previously in Section 2.6, however, this laser material has the lowest thermal and stress figures-of-merit (Table 3), potentially limiting its usefulness as a material for high average power ultrafast systems. As shown in Figure 15, even for modest $\mathrm{Yb}$ doping levels of a few at. \%, the thermal conductivity of $\mathrm{Yb}: \mathrm{CaF}_{2}$ can be less than the undoped value at $300 \mathrm{~K}$ at temperatures of both 300 and $80 \mathrm{~K}$. As a consequence, the figures of merit are even worse for $\mathrm{Yb}$-doped $\mathrm{CaF}_{2}$ crystals. Recall that the figures-of-merit shown in Table 3 are for undoped crystals only. An attractive feature of this material for systems applications is that it has a very low nonlinear index (Table 4), although such a low value makes Kerr-lens mode-locking much more difficult.

For the sesquioxide laser materials at room temperature, the expected Gaussian transform-limited pulsewidths are calculated to be 119, 119, and 109 fs for $\mathrm{Yb}: \mathrm{Lu}_{2} \mathrm{O}_{3}, \mathrm{Yb}: \mathrm{Sc}_{2} \mathrm{O}_{3}$, and $\mathrm{Yb}: \mathrm{Y}_{2} \mathrm{O}_{3}$ respectively. All have relatively good thermal and stress figures-of-merit (Table 3).

\section{Hydrothermal Crystal Growth}

In this section of our paper, we review the substantial progress we have made utilizing the hydrothermal method of crystal growth, and in particular in the growth of sesquioxide laser materials. Interested readers may also consult two recent publications by our group [104,112]. The rare earth sesquioxides, $\mathrm{Sc}_{2} \mathrm{O}_{3}, \mathrm{Y}_{2} \mathrm{O}_{3}$, and $\mathrm{Lu}_{2} \mathrm{O}_{3}$, have emerged as extremely attractive optical materials, particularly for their potential as high power laser materials. They all possess relatively high thermal conductivities, high thermal stability, suitably broad absorption bands, a wide transparency range, and a cubic crystal structure, all of which are very appealing characteristics for solid-state laser hosts. In particular, $\mathrm{Lu}_{2} \mathrm{O}_{3}$ appears especially promising since $\mathrm{Lu}^{3+}$ offers a close size and mass match to many of the lanthanide dopant ions of interest, allowing the crystals to support high doping levels with minimal sacrifice of thermal conductivity (see Figure 6). Several recent studies have confirmed the potential of lanthanide doped $\mathrm{Lu}_{2} \mathrm{O}_{3}$ as a versatile high power optical material $[113,114]$. As such they make very attractive targets for single crystal growth studies.

The high melting point of $\mathrm{Lu}_{2} \mathrm{O}_{3}\left(2450^{\circ} \mathrm{C}\right)$ presents significant technical challenges to the growth of single crystals by classical melt-based methods. Few traditional approaches have gained traction, and recent efforts in $\mathrm{Lu}_{2} \mathrm{O}_{3}$ crystal growth have converged on a few techniques. The micro-pulling-down method has been used to grow single crystals a few millimeters in diameter with lanthanide doping targeted for laser applications [115]. The extreme crystal growth demands and promising materials characteristics of the sesquioxide systems prompted us to develop a unique hydrothermal approach to the growth of $\mathrm{Sc}_{2} \mathrm{O}_{3}$ and $\mathrm{Lu}_{2} \mathrm{O}_{3}$ [112]. Hydrothermal crystal growth of these materials can occur in a closed system at modest temperatures $\left(550-650^{\circ} \mathrm{C}\right)$, well below the melting point of the oxides using a supercritical aqueous hydroxide solution as a transport medium. Many refractory oxides are soluble under these conditions, and growth can occur either by spontaneous nucleation, or be deposited onto a seed crystal. The much lower temperature hydrothermal growth of $\mathrm{Yb}: \mathrm{Lu}_{2} \mathrm{O}_{3}[112,116]$ and other sesquioxide materials results in the minimization of defects, impurities, crystal strain, color-centers, and inhomogeneities, all of which are important issues in melt-based growth methods. Comprehensive reviews of the hydrothermal growth process and its application to the development of new and promising laser crystals have recently been published [116-118]. In earlier work [104] we presented the first room temperature absorption cross-section data for hydrothermally-grown $\mathrm{Yb}: \mathrm{Lu}_{2} \mathrm{O}_{3}$, as well as the emission cross-sections determined by the method of reciprocity. An image of a recently grown, faceted, large $\mathrm{Yb}: \mathrm{Lu}_{2} \mathrm{O}_{3}$ crystal is shown in Figure 34 . 


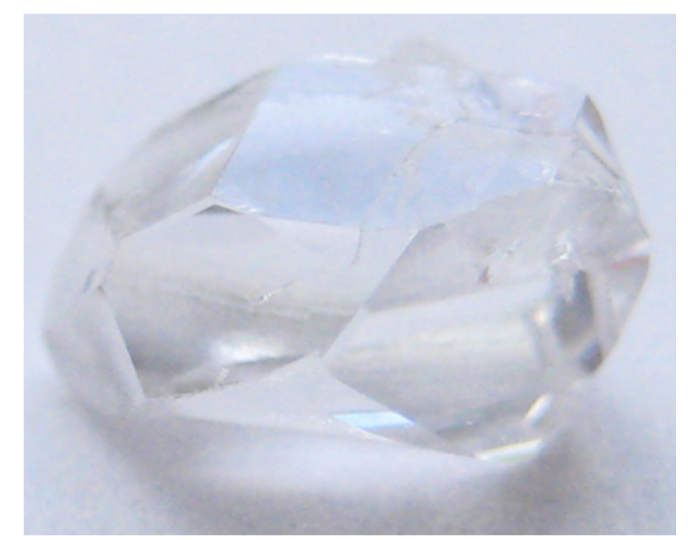

Figure 34. Highly faceted, optically clear, as-grown $\mathrm{Yb}: \mathrm{Lu}_{2} \mathrm{O}_{3}$ single crystal grown at $700{ }^{\circ} \mathrm{C}$ with a 30 degree thermal gradient. Crystal dimensions are $6 \times 6 \times 4 \mathrm{~mm}^{3}$.

\subsection{Recent Results For Sesquioxide Laser Materials}

\subsection{1. $\mathrm{Yb}: \mathrm{Lu}_{2} \mathrm{O}_{3}$ Absorption Spectroscopy}

Our group has recently obtained cryogenic absorption cross-section data for $\mathrm{Yb}: \mathrm{Lu}_{2} \mathrm{O}_{3}$, with the results submitted for publication [119]. Figure 35 shows a portion of that data at $298 \mathrm{~K}$ and $80 \mathrm{~K}$. These data were obtained with high spectral resolution down to $0.1 \mathrm{~nm}$, and clearly show that the zero-phonon line is still not resolved at $80 \mathrm{~K}$. While the absorption band around $900 \mathrm{~nm}$ is resolved at all temperatures, and we see the usual progression of increased cross-section values as temperature is lowered, the bands at $950 \mathrm{~nm}$ and $977 \mathrm{~nm}$ are not completely resolved, even at $0.1 \mathrm{~nm}$ resolution. Our analysis shows that the zero-phonon linewidth is $<0.1 \mathrm{~nm}$ and likely below $0.05 \mathrm{~nm}$. A similar situation has been found in Yb:YAG where the zero-phonon linewidth was initially measured to be $<0.1 \mathrm{~nm}$ [27], and a later measurement yielded a value of $0.05 \mathrm{~nm}$ [3]. Due to the narrow linewidth, it is unlikely that the lower quantum defect associated with the $977 \mathrm{~nm}$ line can be obtained in practice, since the linewidth needed for efficient optical pumping is beyond even the capabilities of recently developed volume-Bragg-grating (VBG) narrowed diode laser sources. For the $950 \mathrm{~nm}$ band, however, there is an increased cross-section at $80 \mathrm{~K}$, however, when higher-resolution measurements are available the increase will likely be larger than shown in Figure 35.

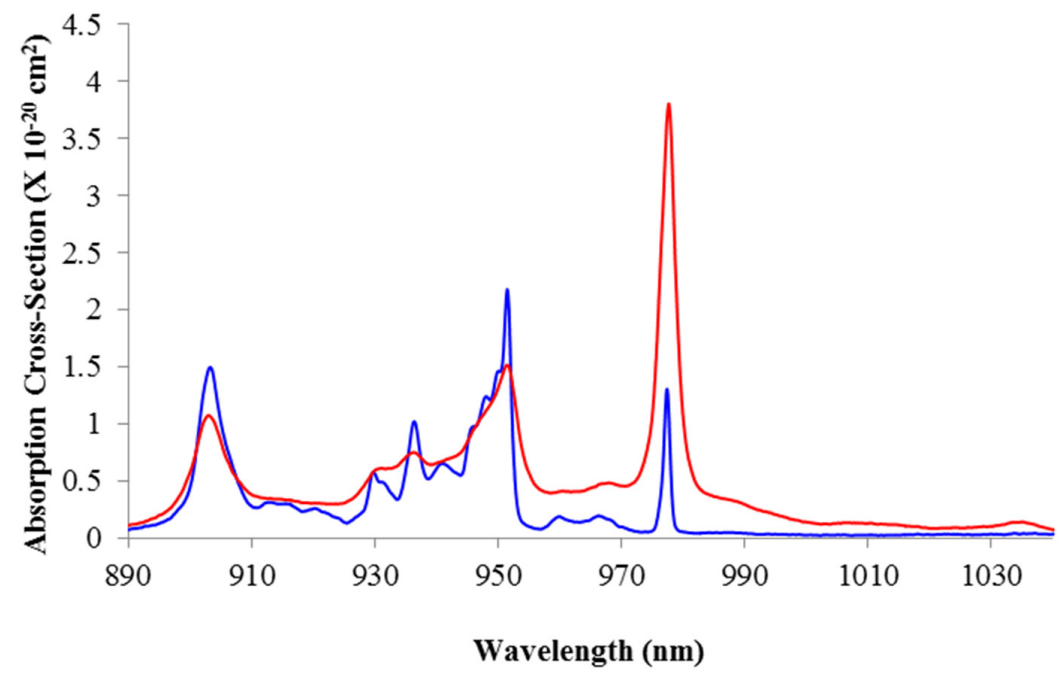

Figure 35. Absorption cross-section as a function of wavelength for a hydrothermally-grown $\mathrm{Yb}^{3+}: \mathrm{Lu}_{2} \mathrm{O}_{3}$ laser crystal at $80 \mathrm{~K}$ (blue) and $298 \mathrm{~K}$ (red). 
3.1.2. Sm:YAG and Sm:LuAG For The Suppression of Parasitic Oscillations and Amplified Spontaneous Emission

We have investigated the hydrothermal growth of both Sm:YAG and Sm:LuAG. Sm is a well-known $1064 \mathrm{~nm}$ absorber, and has been used as Sm:Glass and Sm:YAG for some time as a suppressor of parasitic oscillations and amplified spontaneous emission (ASE). Figure 36 shows a side view of a Nd:YAG laser rod with an external Sm:YAG cladding layer on the barrel. In the absence of the Sm:YAG coating, it is well-known that a polished barrel will result in the oscillation of transverse straight-across surface mode, ring modes, and longitudinal (bulk) parasitic oscillation modes that will clamp the laser gain. In some cases, a surface parasitic located at the pump beam entrance face will have the lowest threshold. To eliminate parasitics and minimize ASE, such rods usually have ground barrels. Grinding of course results in sub-surface fractures that limit the power handling capability of the rod. A highly-doped layer of Sm:YAG can be used to suppress the parasitics and minimize ASE losses.

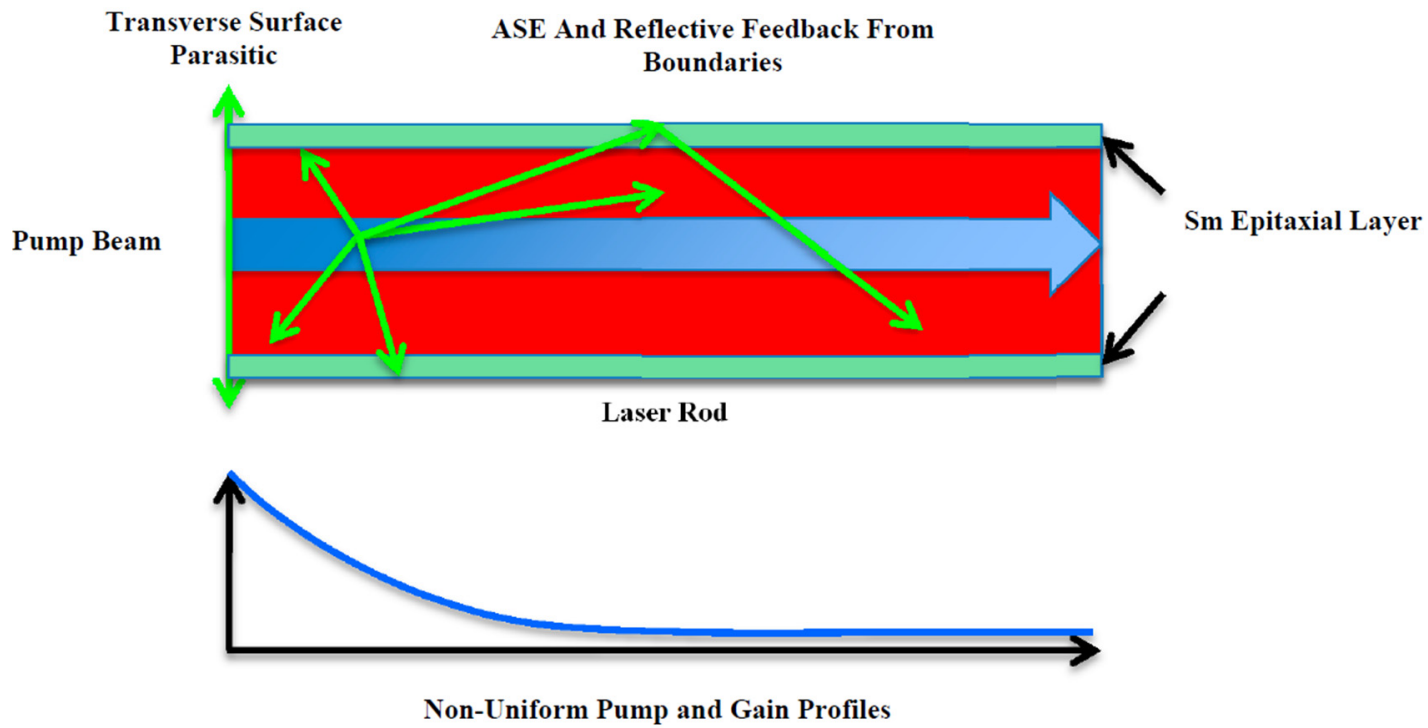

Figure 36. Side view of an end-pumped laser rod or slab, showing the emitted fluorescence and absorption in Sm:YAG cladding layer. The non-uniform absorption gives rise to a possible surface parasitic at the pump beam entrance face where the transverse gain is largest.

Our interest in these materials began in 2012 when we demonstrated, for the first time, that we could grow epitaxial-like layers of Sm:YAG on Nd:YAG substrates [120]. A more recent example of this is shown in Figure 37. A Nd-doped rectangular $\mathrm{cm}$ sized laser crystal was used, and a highly doped layer of Sm:YAG was successfully grown on all six external surfaces. After layer growth, the end faces of the crystal are ground to remove the Sm:YAG, and polished and dielectrically-coated to allow $1064.2 \mathrm{~nm}$ lasing. In practice, this type of layer may be grown on any Nd:YAG laser crystal, and can be used to suppress parasitics and ASE. Previously, other researchers have reported Sm:YAG clad ceramic Nd:YAG rods [121,122] and disks [123]. In our work, we are primarily interested in providing a method that can be use on commonly used Czochralski-grown single crystals, without using expensive methods like diffusion-bonding.

Figures 38 and 39 show the absorption cross-sections as a function of wavelength for Sm:YAG and Sm:LuAG. We are particularly interested in the region near $1064 \mathrm{~nm}$. To look at the potential of these materials as parasitics and ASE suppressors, we have compared the two materials in Figure 40, where we show the normalized cross-sections as a function of wavelength from 1050 to $1100 \mathrm{~nm}$. The cross-section values for Sm:YAG and Sm:LuAG are nearly identical at 1064.2 (Nd:YAG) and 
1064.4 nm (Nd:LuAG). Sm:YAG and Sm:LuAG, which may be grown as epitaxial layers on Nd:YAG, and Nd:LuAG, will then be effective parasitic and ASE absorbers for those materials.

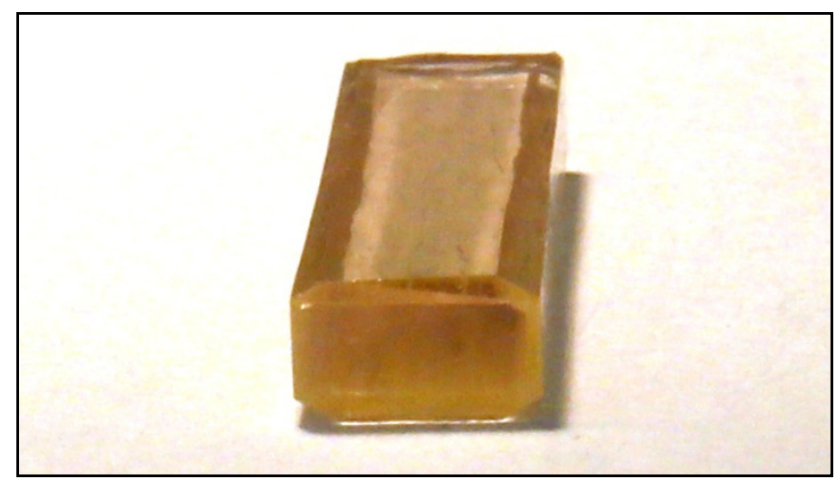

Figure 37. Rectangular Nd:YAG crystal with hydrothermally-grown Sm:YAG layer for parasitics and amplified-spontaneous-emission suppression. After the growth of the layer, the Sm:YAG is removed from the end faces by polishing and the surfaces dielectric coated.

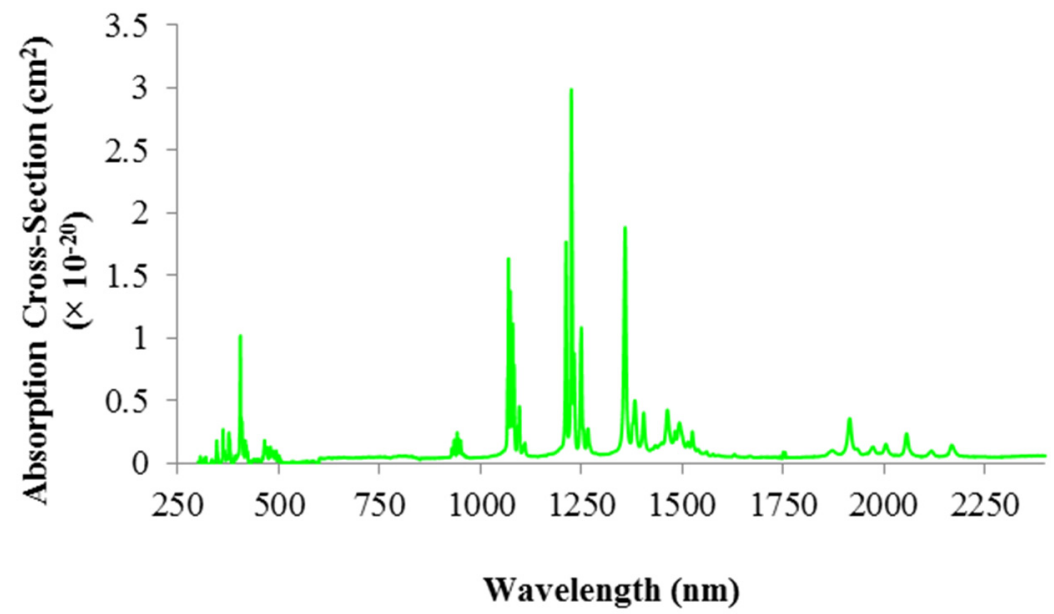

Figure 38. Absorption cross-section as a function of wavelength for a hydrothermally-grown $\mathrm{Sm}^{3+}$ :YAG crystal at $298 \mathrm{~K}$.

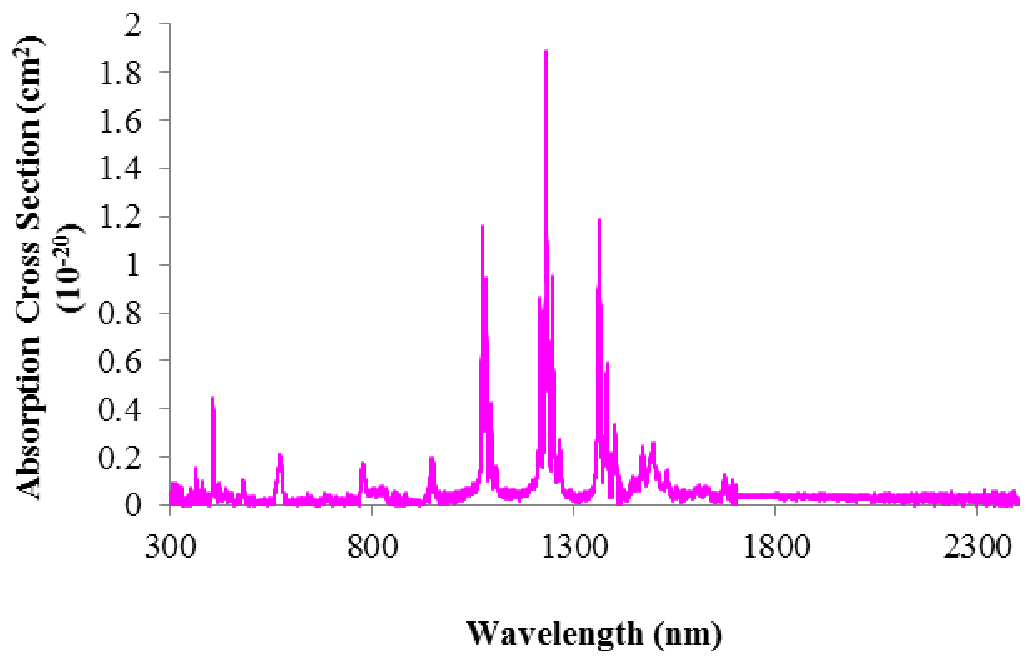

Figure 39. Absorption cross-section as a function of wavelength for a hydrothermally-grown $\mathrm{Sm}^{3+}:$ LuAG crystal at $298 \mathrm{~K}$. 


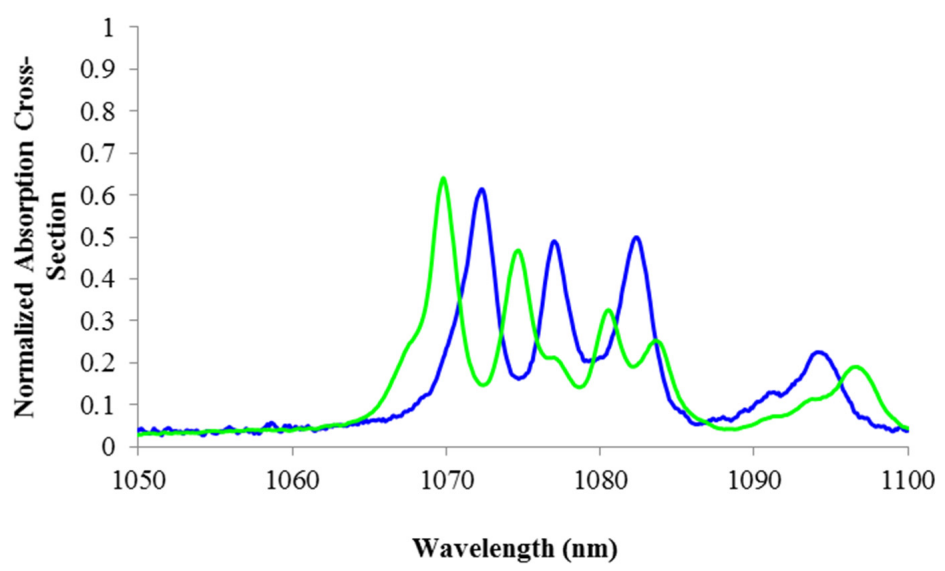

Figure 40. Normalized absorption cross-section as a function of wavelength in the region from 1050 to $1100 \mathrm{~nm}$, for the Sm-doped materials Sm:YAG (green) and Sm:LuAG (blue).

Figure 41 shows the transmission of $1064.2 \mathrm{~nm}$ fluorescence from Nd:YAG through a Sm:YAG doped layer as a function of optical thickness, defined here as the Sm doping density times the penetration depth. With 5 at. \% Sm doping and a thickness of $3 \mathrm{~mm}$, the optical thickness is about $1 \times 10^{19} / \mathrm{cm}^{2}$ ). From Figure 41 we see that the transmission is about 0.85 for a single pass, and the absorption for normal incidence rays is about $15 \%$. For a normal incidence reflection from the rod barrel, the reflection coefficient is $8.5 \%$; a return ray makes two passes of the Sm layer, and the return intensity is only about $6 \%$. By increasing the doping level even further, to 15 at. \%, the one-way transmission if reduced to $\sim 65 \%$, so for a return normal incidence ray the intensity is only about $0.4 \%$. Since many ring parasitics and bulk parasitics, however, have incident angles much larger than $90^{\circ}$, the absorption depth is much longer, and even relatively low doping levels can be effective in suppressing parasitics. The use of low doping levels does allow reflections from the barrel surface to be returned to the gain region of the rod and increase the ASE level, although if the barrel is in contact with water, the returned ASE is much less. We have successfully doped Sm into YAG with $>15$ at. \% concentration.

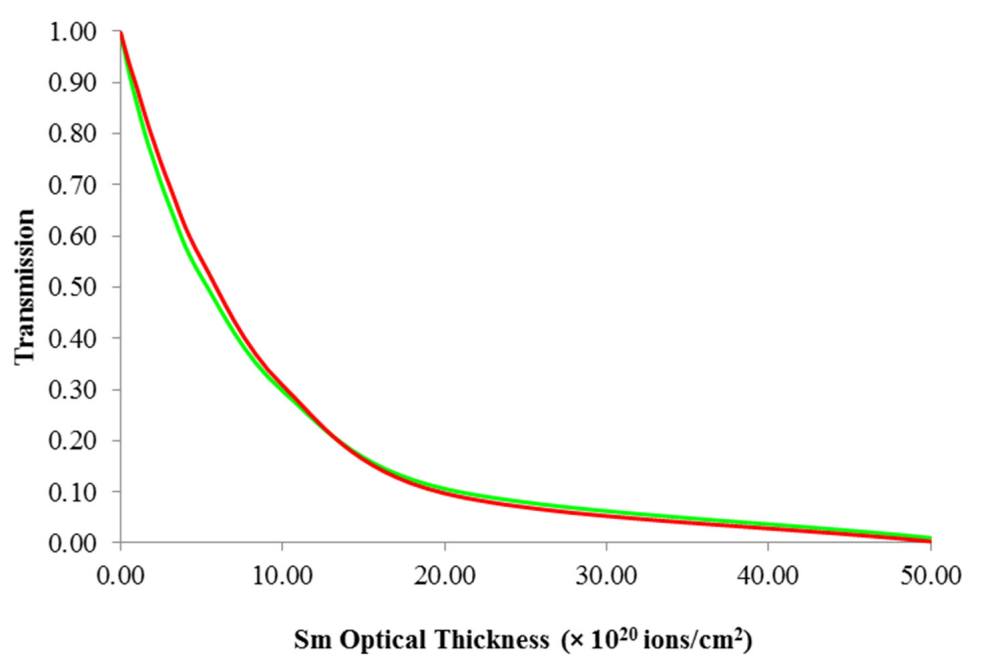

Figure 41. Transmission of Nd:YAG 1064.2 fluorescence through a Sm:YAG layer, as a function of optical thickness (Sm doping density—Sm:YAG thickness product), for a Nd:YAG FWHM gain bandwidth of $1.1 \mathrm{~nm}$, and assuming a Gaussian (red) or Lorentzian (green) gain spectral profile.

An attractive feature of the Sm:YAG and Sm:LuAG spectra is that they have little absorption around $808 \mathrm{~nm}$, the main pumping channel used for Nd:YAG. In that regard, for Nd:YAG and $\mathrm{Nd}: L u A G$ Sm:YAG and Sm:LuAG are superior to $\mathrm{Cr}^{4+}:$ YAG which has substantial absorption at that 
wavelength and can be partially bleached by the pump diodes. That consideration is particularly important for transverse-pumping geometries.

For higher average power lasers, placing a Sm absorbing layer directly on the outside surface of a rod or other lasing crystal geometry may not be the optimum solution, because the absorption of high densities of heat in the Sm cladding later will result in complicated radially varying temperature profiles, leading to unwanted stresses in the doped lasing region. The best solution may be to grow an undoped buffer layer between the lasing and absorbing regions, as well as to have a Sm gradient layer whose doping is lowest next to the undoped region and highest at the edge. Hydrothermal growth is capable of producing such gradient doped layers [120].

3.1.3. Dy:YAG and Dy: $\mathrm{Lu}_{2} \mathrm{O}_{3}$ For The Suppression of Parasitic Oscillations and Amplified Spontaneous Emission

We are also investigating hydrothermally-grown Dy:YAG and Dy: $\mathrm{Lu}_{2} \mathrm{O}_{3}$ crystals with a view towards their use as potential parasitics and amplified-spontaneous-emission (ASE) absorbers. The measured absorption cross-section data are shown in Figures 42 and 43 for Dy:YAG and $\mathrm{Dy}: \mathrm{Lu}_{2} \mathrm{O}_{3}$ respectively. Dy:YAG is an interesting laser material that has received attention recently as a relatively rare example of a direct transition $\left({ }^{4} \mathrm{~F}_{9 / 2}{ }^{-6} \mathrm{H}_{13 / 2}\right)$ in the yellow spectral region at $583 \mathrm{~nm}$ [124]. Previously, $\mathrm{Sm}^{3+}, \mathrm{Co}^{2+}, \mathrm{Co}^{3+}, \mathrm{Cr}^{3+}$, and $\mathrm{Cr}^{4+}$ in YAG have been evaluated as parasitic and ASE absorbers for ceramic laser materials at the Nd:YAG wavelength, $1064 \mathrm{~nm}$ [125]. Our interest is in developing parasitic and ASE absorbers that are effective at various $\mathrm{Yb}$ wavelengths around $1030 \mathrm{~nm}$, and that are effective at room temperature and cryogenic temperatures. In particular, we are interested in absorbers that are effective and can be epitaxially-grown on the outside surfaces of $\mathrm{Yb}: \mathrm{YAG}$ and $\mathrm{Yb}: \mathrm{Lu}_{2} \mathrm{O}_{3}$ laser elements. For Dy:YAG, the absorption cross-sections at $1030 \mathrm{~nm}$ are rather small, $6 \times 10^{-23} \mathrm{~cm}^{2}$ at $298 \mathrm{~K}$, and $9 \times 10^{-23} \mathrm{~cm}^{2}$ at $80 \mathrm{~K}$. Even for large Sm dopings, as a parasitics and ASE absorber this material seems marginal at best. In the case of $\mathrm{Dy}: \mathrm{Lu}_{2} \mathrm{O}_{3}$, however, the results are more promising, with the absorption cross-sections at $1033 \mathrm{~nm}$ are $6 \times 10^{-22} \mathrm{~cm}^{2}$ at $298 \mathrm{~K}$, and $2 \times 10^{-22} \mathrm{~cm}^{2}$ at $80 \mathrm{~K}$. These cross-sections are roughly an order of magnitude lower than for Sm:YAG at $1064 \mathrm{~nm}$, however, larger Sm doping densities can make this crystal useful at room and cryogenic temperatures. It is however likely that for Yb:YAG, either $\mathrm{Cr}^{4+}:$ YAG or $\mathrm{Co}^{3+}$ :YAG may be better choices since the absorption cross-sections in those materials, both of which may be epitaxially grown on Yb:YAG using the hydrothermal method, are much larger at $1029 \mathrm{~nm}$. A similar conclusion may be reached if $\mathrm{Cr}^{4+}$ and $\mathrm{Co}^{3+}$ are doped into $\mathrm{Lu}_{2} \mathrm{O}_{3}$.

A feature of Figures 42 and 43 worth noting is that the absorption cross-sections around $1300 \mathrm{~nm}$ are large, and Dy:YAG can be an effective suppressor of ASE from the 1319 and $1338 \mathrm{~nm}$ transitions in Nd:YAG. Similarly, Dy: $\mathrm{Lu}_{2} \mathrm{O}_{3}$ can suppress the $1359 \mathrm{~nm}$ transition in $\mathrm{Nd}: \mathrm{Lu}_{2} \mathrm{O}_{3}$. At $946 \mathrm{~nm}$, both Dy:YAG and $\mathrm{Dy}: \mathrm{Lu}_{2} \mathrm{O}_{3}$ have marginal absorption.

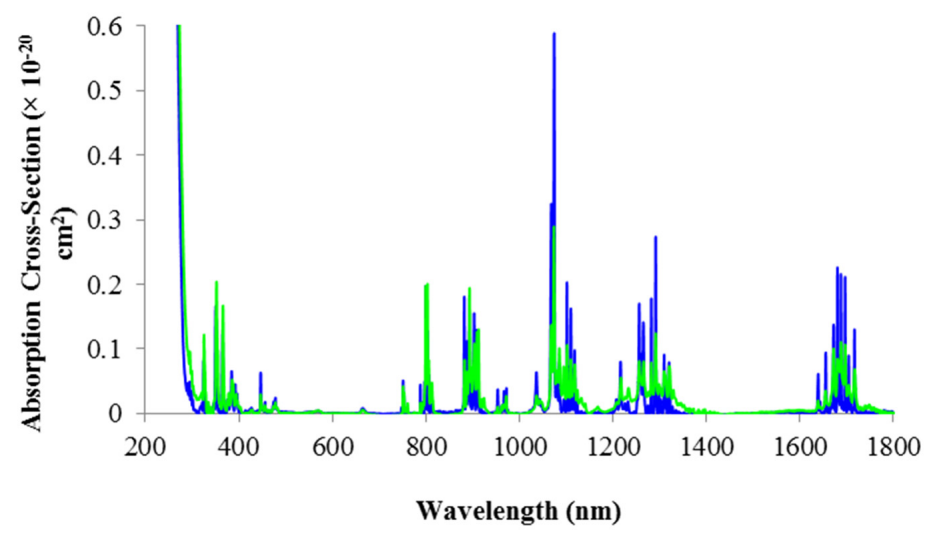

Figure 42. Absorption cross-section as a function of wavelength for a hydrothermally-grown $\mathrm{Dy}^{3+}$ :YAG crystal at $80 \mathrm{~K}$ (blue) and $298 \mathrm{~K}$ (green). 


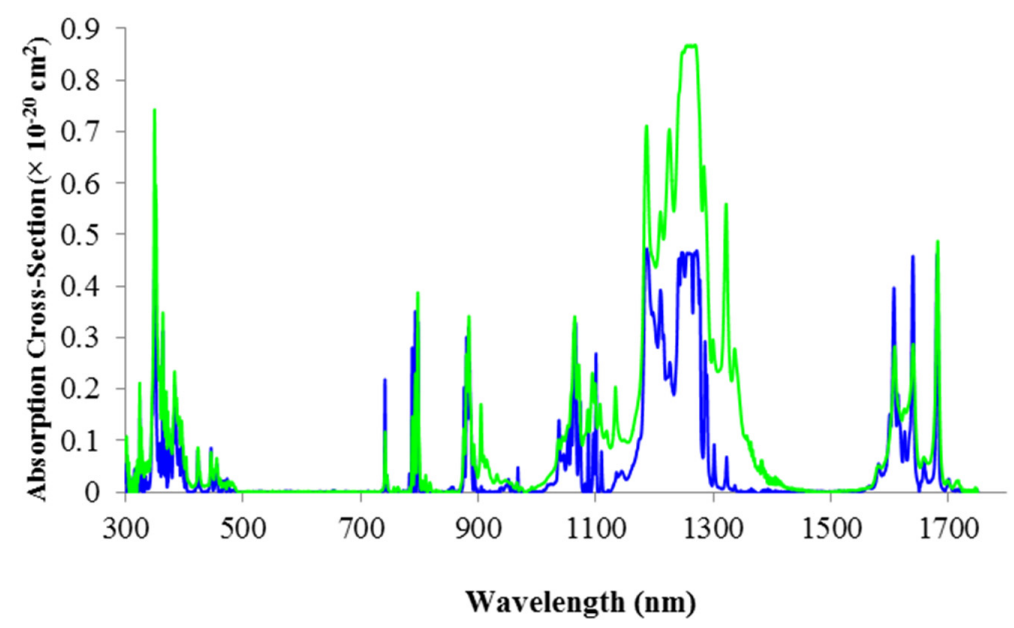

Figure 43. Absorption cross-section as a function of wavelength for a hydrothermally-grown $\mathrm{Dy}^{3+}: \mathrm{Lu}_{2} \mathrm{O}_{3}$ crystal at $80 \mathrm{~K}$ (blue) and $298 \mathrm{~K}$ (green).

\section{Review and Status of Cryogenic Laser Technology}

In the past ten years, enormous progress has been made in our fundamental understanding of cryogenic lasers. Our increased knowledge of the fundamental materials properties of laser crystals at cryogenic temperatures has enabled the demonstration of ever more powerful cryogenic laser sources. The already demonstrated compelling performance advantages of cryogenic lasers has led to the adoption of the technology by many researchers around the world, and have garnered attention in the commercial and military laser communities as well. While the acceptance of cryogenic lasers in commercial and military applications has been delayed, due in large part to the need for the acceptance of cryogens that add complexity and cost, the technology has now been fully embraced by the scientific community because of the many performance advantages that can be obtained, and the need for HAPP lasers with near-diffraction-limited beam quality.

\subsection{Cryogenic CW Lasers}

In this section of the manuscript we will review the progress that has been made in the development of $\mathrm{CW}$, nanosecond, picosecond, and femtosecond cryogenic laser sources in the modern era. We begin by first looking at the evolution of $\mathrm{Yb}: \mathrm{YAG}, \mathrm{Yb}: \mathrm{YLF}$, and $\mathrm{Yb}: \mathrm{CaF}_{2} \mathrm{CW}$ cryogenic lasers; $Y b: Y A G$ and $Y b$ :YLF materials have been used for the highest power $C W$ demonstrations to date. Figure 44 shows the $\mathrm{CW}$ output power obtained from Yb:YAG cryogenically-cooled lasers operating near $80 \mathrm{~K}$. The corresponding data and references are shown in Table $7[1,3,4,30,108,109,126-139]$. The first CW Yb:YAG demonstration was by Lacovara [1], who reported $40 \mathrm{~W}$ output power. The earliest demonstration of CW power in excess of $100 \mathrm{~W}$ is attributed to [109], whom reported a cryogenic Yb:YLF laser with output power of $110 \mathrm{~W}$. This was followed by two early high power CW Yb:YAG cryogenic laser demonstrations at $165 \mathrm{~W}$ [126] and $300 \mathrm{~W}$ [127] by the MIT cryogenic laser group. $\mathrm{CW}$ cryogenic Yb:YAG lasers with $\mathrm{CW}$ output power $\geqslant 400 \mathrm{~W}$ have been reported by a number of groups, including $455 \mathrm{~W}$ [3], $400 \mathrm{~W}$ [129], $550 \mathrm{~W}$ [130], $500 \mathrm{~W}$ [135], and $494 \mathrm{~W}$ [138]. The largest CW power for a Yb:YAG cryogenic laser reported to date, $963 \mathrm{~W}$, was for a Yb:YAG cryogenic oscillator driven double-passed Yb:YAG preamplifier followed by a Yb:YAG power amplifier demonstrated by our group [132]. We include $\mathrm{Yb}: \mathrm{CaF}_{2}$ here due to a recent high power demonstration with a quantum-defect of only $1.11 \%$ [134].

For $\mathrm{Yb}: \mathrm{YLF}$, the highest average power reported to date is $180 \mathrm{~W}$ by the MIT cryogenic laser group [108]. We note that almost all of the CW laser sources included in Figure 44 had near-diffraction-limited beam-quality with $\mathrm{M}^{2}$ values typically $<1.3$. The one exception is [129], which reported multi-transverse-mode operation. 


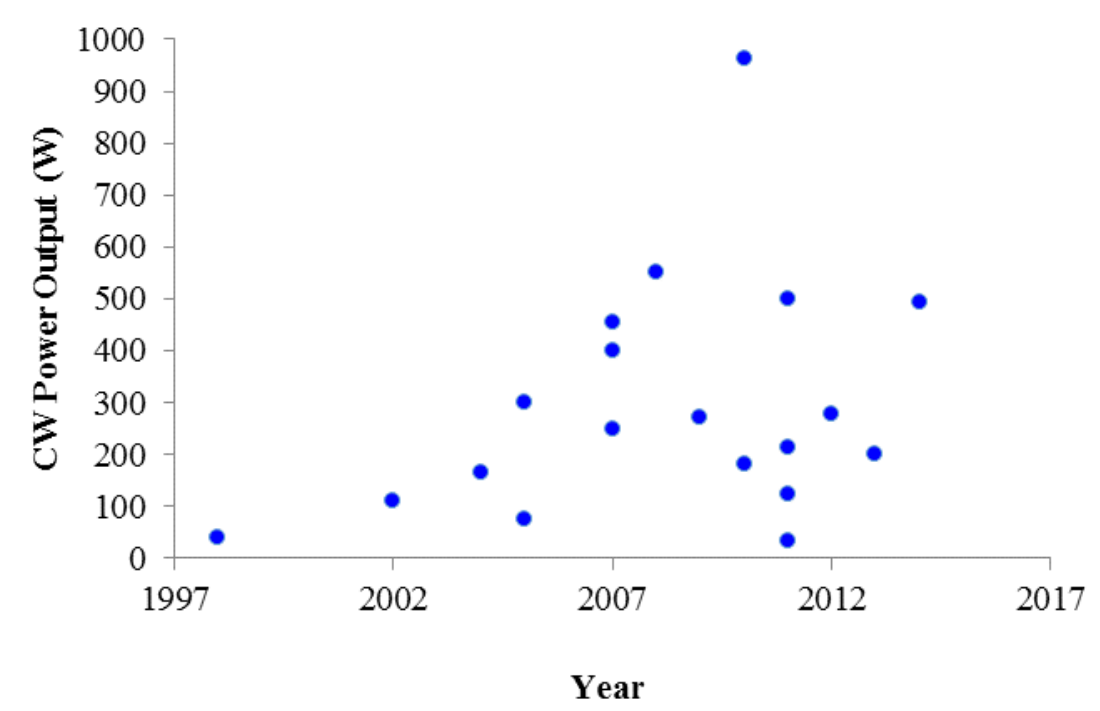

Figure 44. CW output power at $1029 \mathrm{~nm}$ from demonstrated Yb:YAG and Yb:YLF cryogenic lasers in the years 1998-2015.

Table 7. Reported Cryogenic CW Yb:YAG and Yb:YLF Demonstrations From 1998-2015.

\begin{tabular}{cccc}
\hline Year & CW Output Power $(\mathbf{W})$ & Laser Material & Reference \\
\hline 1998 & 40 & Yb:YAG & {$[1]$} \\
2001 & 110 & Yb:YLF & {$[109]$} \\
2004 & 165 & Yb:YAG & {$[126]$} \\
2005 & 300 & Yb:YAG & {$[127]$} \\
2005 & 75 & Yb:YAG & {$[128]$} \\
2007 & 250 & Yb:YAG & {$[129]$} \\
2007 & 455 & Yb:YAG & {$[3]$} \\
2007 & 400 & Yb:YAG & {$[4]$} \\
2008 & 550 & Yb:YAG & {$[130]$} \\
2009 & 273 & Yb:YAG & {$[131]$} \\
2010 & 180 & Yb:YLF & {$[108]$} \\
2010 & 973 & Yb:YAG & {$[132]$} \\
2011 & 214 & Yb:YAG & {$[133]$} \\
2011 & 123 & Yb:YAG & {$[30]$} \\
2011 & 33 & Yb:CaF & {$[134]$} \\
2011 & 500 & Yb:YAG & {$[135]$} \\
2012 & 277 & Yb:YAG & {$[136]$} \\
2013 & 200 & Yb:YAG & {$[137]$} \\
2014 & 494 & Yb:YAG & {$[138,139]$} \\
\hline
\end{tabular}

\subsection{Other Cryogenic CW Lasers}

\subsubsection{Ho:YAG Cryogenic Lasers}

Other cryogenic CW lasers have been developed recently as well. In [140], the authors describe a cryogenically-cooled CW Ho:YAG laser pumped by a Tm:Glass fiber laser with output at $1932 \mathrm{~nm}$, producing $8 \mathrm{~W}$ of CW power output at $\sim 2100 \mathrm{~nm}$. In addition, still pumping at $1932 \mathrm{~nm}$, the authors report $2.5 \mathrm{~W}$ of $\mathrm{CW}$ output power at $1976 \mathrm{~nm}$, resulting in a very low quantum defect of $2.2 \%$.

\subsubsection{Er Cryogenic Lasers}

A number of Er based cryogenic lasers have also been reported in the literature [141-147]. A group at the U.S. Army Research Laboratory has investigated $\mathrm{Er}_{\mathrm{Y}} \mathrm{Y}_{2} \mathrm{O}_{3}$ [141,142], Er: $\mathrm{Sc}_{2} \mathrm{O}_{3}$ [144], and Er:YAG $[144,145]$ crystals operated at cryogenic temperatures as potential candidates as eye-safe 
lasers. In [144], a $1530 \mathrm{~nm}$ pumped Er:YAG crystal at $78 \mathrm{~K}$ was operated quasi-CW, producing $375 \mathrm{~mJ}$ energy/pulse at a $2 \mathrm{~Hz}$ repetition rate. The output wavelength was $1618 \mathrm{~nm}$ for temperatures $<90 \mathrm{~K}$, and $1645 \mathrm{~nm}$ for $>110 \mathrm{~K}$. Further results for Er:YAG were later reported in [145], where a careful and thorough study of the spectroscopic and lasing properties of the material are discussed. Over $25 \mathrm{~W}$ of quasi-CW output power was obtained. A $1525 \mathrm{~nm}$ pumped $\mathrm{Er} \mathrm{Sc}_{2} \mathrm{O}_{3}$ crystal was demonstrated to operate at $1558 \mathrm{~nm}$, resulting in a very low quantum defect of only $1.5 \%$ [143]. The CW output power was $3.3 \mathrm{~W}$. An Er: $\mathrm{Y}_{2} \mathrm{O}_{3}$ crystal diode-pumped with a $974 \mathrm{~nm}$ source was also demonstrated with output at $2710 \mathrm{~nm}$ and CW output of $14 \mathrm{~W}$ [142]. One higher average power demonstration has been discussed in the literature as well, at cryogenic temperatures. A $400 \mathrm{~W}$ Er:YAG cryogenic laser has been described [146] that produced $386 \mathrm{~W}$ of $\mathrm{CW}$ output power at $1645 \mathrm{~nm}, 420 \mathrm{~W}$ of quasi-CW power, and was also operated Q-switched. Most of the results described here were obtained in "proof-of-principle experiments", using relatively recently developed InGaAsP/InP (1000-1650 nm) diode laser sources that are not as technologically or commercially as well-developed as InGaAs/GaAs and InGaAs/GaAs diode sources operating in the 720-850 nm and 900-1100 nm ranges respectively. As a consequence, beam-quality is seldom mentioned, and that the transverse mode distributions are multi-mode. As discussed in the aforementioned, most Yb:YAG cryogenic laser sources routinely produce $M^{2}$ values of $<1.3$, and the transverse mode-structure is a single Gaussian mode. This is largely due to the availability of more advanced high-brightness diode sources.

Figure 45 shows CW and QCW power output against the year demonstrated. The data and references are summarized in Table 8 . While undoubtedly higher CW power outputs will be reported in the future to address a number of commercial and military applications, at the present time most cryogenic laser research is centered on the development of high-average-power nanosecond, picosecond, and femtosecond laser sources.

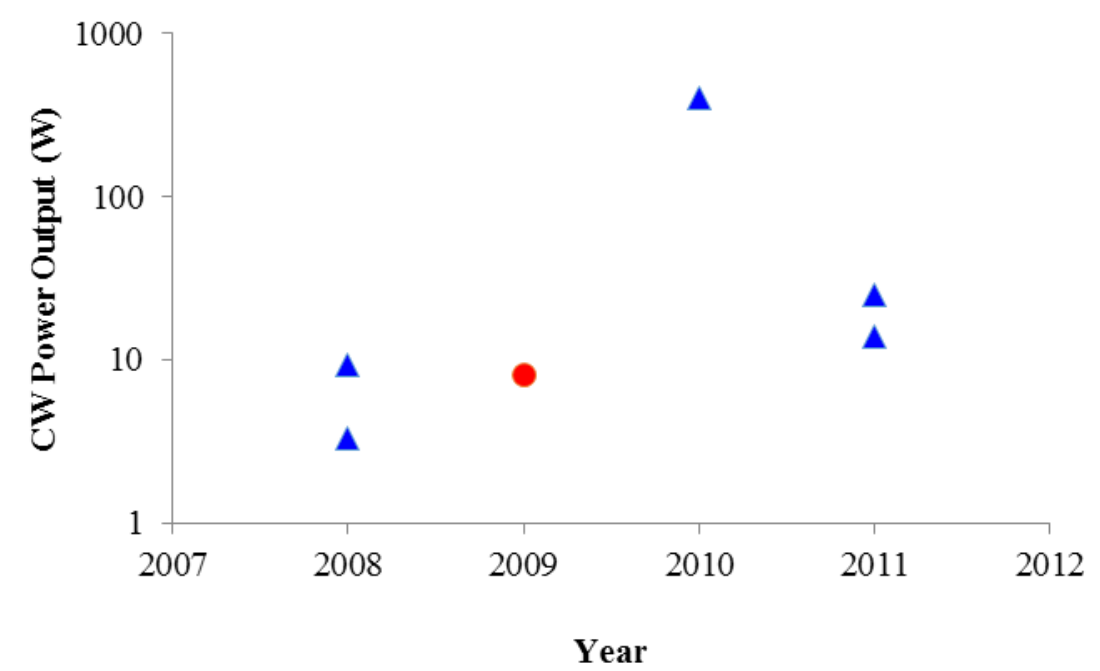

Figure 45. CW and QCW output powers from Er (blue) and Ho:YAG (red) cryogenic lasers from 2008 through 2011.

Table 8. Reported Cryogenic CW Er and Ho Demonstrations From 2005-2011.

\begin{tabular}{cccc}
\hline Year & CW Output Power $(\mathbf{W})$ & Laser Material & Reference \\
\hline 2008 & 9.3 & $\mathrm{Er:}_{2} \mathrm{O}_{3}$ & {$[141]$} \\
2008 & 3.3 & $\mathrm{Er}: \mathrm{Sc}_{2} \mathrm{O}_{3}$ & {$[143]$} \\
2009 & 8 & $\mathrm{Ho}: Y A G$ & {$[140]$} \\
2010 & 400 & $E r: Y A G$ & {$[146]$} \\
2011 & 25 & Er:YAG & {$[144]$} \\
2011 & 14 & Er: $\mathrm{Y}_{2} \mathrm{O}_{3}$ & {$[142]$} \\
\hline
\end{tabular}




\subsection{Cryogenic Nanosecond Lasers}

In this section of the manuscript we review the performance of nanosecond cryogenic laser technology. Figure 46 displays the energy/pulse, repetition rate as a function of average power. Table 9 tabulates the corresponding date, energy/pulse, repetition rate, average power, pulsewidth, and reference [30,135,147-153]. A number of techniques are used to generate nanosecond pulses from cryogenic amplifiers, including acousto-optic or electro-optic Q-Switching, seeded regenerative amplifiers, and in some case regenerative amplifiers followed by one or more power amplifier stages. In [147], $6.5 \mathrm{~mJ} /$ pulse at $200 \mathrm{~Hz}$ and a $10 \mathrm{~ns}$ FWHM pulsewidth, $1.5 \mathrm{~mJ}$ at $1 \mathrm{kHz}$ with a $10 \mathrm{~ns}$ pulsewidth, and $140 \mathrm{~mJ}$ at $100 \mathrm{~Hz}$ with a $10 \mathrm{~ns}$ pulsewidth were reported, respectively. For this system, a seed laser was used to drive a regenerative amplifier that in turn drove a 4-pass power amplifier. The highest average power produced was $14 \mathrm{~W}$. An earlier paper [148] reported the demonstration of $4.6 \mathrm{~mJ}$ pulse energy at a $16 \mathrm{kHz}$ repetition rate in an oscillator configuration that incorporated an acousto-optic Q-switch. The average power produced was $74 \mathrm{~W}$ and the pulsewidth $175 \mathrm{~nm}$. Another lower average power cryogenic laser that produced $120 \mathrm{~mJ} /$ pulse at a $500 \mathrm{~Hz}$ repetition rate, $60 \mathrm{~W}$ of average power, and a $70 \mathrm{~ns}$ pulsewidth is reported in [149] where a cryogenic Yb:YAG oscillator was used to drive a power amplifier. The cryogenic laser group at MIT, the first to report average power $>100 \mathrm{~W}$, demonstrated a $114 \mathrm{~W}$ average power cryogenic oscillator $[30,150]$ that produced $22.8 \mathrm{~mJ} /$ pulse at $5 \mathrm{kHz}$, and with a pulsewidth of $16 \mathrm{~ns}$. The highest average power nanosecond cryogenic laser reported to date [135] produced $8.5 \mathrm{~mJ}$ pulses at a $40 \mathrm{kHz}$ repetition rate for an average power of $340 \mathrm{~W}$. The pulsewidth produced using an acousto-optic Q-switch was $75 \mathrm{~ns}$.

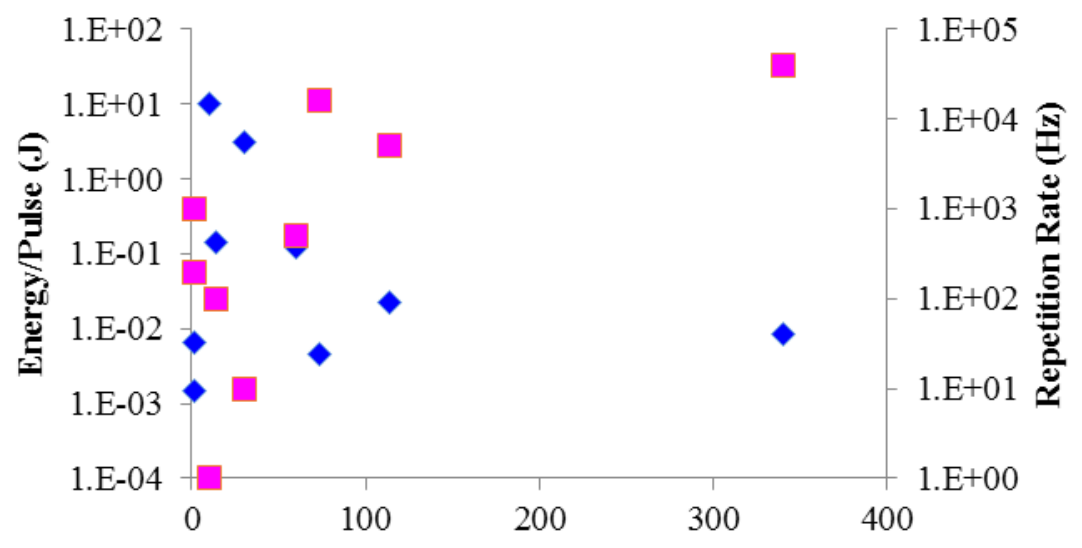

Average Power (W)

Figure 46. Nanosecond output energy/pulse (blue) and repetition rate (pink) as a function of average power at $1029 \mathrm{~nm}$ from demonstrated Yb:YAG cryogenic lasers in the years 2005-2014.

Table 9. Reported Cryogenic Q-Switched Yb:YAG Demonstrations From 2005-2011.

\begin{tabular}{cccccc}
\hline Year & $\begin{array}{c}\text { Energy/Pulse } \\
(\mathbf{m J})\end{array}$ & $\begin{array}{c}\text { Repetition } \\
\text { Rate (Hz) }\end{array}$ & $\begin{array}{c}\text { Average Power } \\
\mathbf{( W )}\end{array}$ & $\begin{array}{c}\text { Pulsewidth } \\
(\mathbf{n s})\end{array}$ & Reference \\
\hline 2005 & 4.6 & 16,000 & 74 & 175 & {$[148]$} \\
2010 & 6.5 & 5000 & 114 & 15 & {$[147]$} \\
2010 & 1.5 & 200 & 1.3 & 10 & {$[147]$} \\
2010 & 140 & 1000 & 1.5 & 10 & {$[153]$} \\
2010 & 22.8 & 5000 & 114 & 16 & {$[30,150]$} \\
2011 & 4.5 & 40,000 & 340 & 75 & {$[135]$} \\
2013 & 120 & 500 & 80 & 70 & {$[149]$} \\
2013 & 10,100 & 1 & 10.1 & 10 & {$[152]$} \\
2014 & 3050 & 10 & 30.5 & 10 & {$[151]$} \\
\hline
\end{tabular}


Two high energy/pulse, low repetition rate cryogenic nanosecond lasers have been reported in the literature. The first [151] achieved $3.05 \mathrm{~J} /$ pulse at $10 \mathrm{~Hz}$ for an average power of $30.5 \mathrm{~W}$ and with a $10 \mathrm{~ns}$ pulsewidth, using a seeded regenerative amplifier followed by active-mirror pre-amplifier and power amplifier stages. The largest energy/pulse was demonstrated in connection with the DiPOLE (Diode-Pumped Optical Laser Experiment) project [152]; 10.1 J/pulse at a $1 \mathrm{~Hz}$ repetition rate was obtained with a $10 \mathrm{~ns}$ pulsewidth and producing $10.1 \mathrm{~W}$ of average power. The laser can also produce $6.4 \mathrm{~J} /$ pulse at $10 \mathrm{~Hz}$ repetition rate. This laser system is innovative in that it uses cryogenically cooled He gas to remove heat from a parallel array of Yb:YAG laser slabs in an arrangement very reminiscent of the Mercury laser project at Lawrence Livermore National Laboratory (LLNL) in the US, where the concept of using stacks of aerodynamically shaped slab holders originated [154]. While we have not specifically discussed the methods of cryogenic-cooling in this article, here we mention that the use of He at cryogenic temperatures to cool laser crystals is in its infancy. Another innovative way to cool laser disks and slabs has recently been published [155] that uses a static He layer that separates an active-mirror amplifier from a copper plate cooled to $77 \mathrm{~K}$ using liquid nitrogen. This technique can eliminate the soldering of laser disks to high thermal conductivity materials such as $\mathrm{Cu}$ or $\mathrm{CuW}$, and substantially increases the lifetime of HR coatings on active-mirror disks that can be degraded from contact with liquid coolants such as water or liquid nitrogen.

\subsection{Cryogenic Ultrafast (Picosecond and Femtosecond) Lasers}

In this final section of our paper, we discuss the application of cryogenic laser technology to enhancing the performance of ultrafast lasers operating in the picosecond and femtosecond regimes. Cryogenic cooling has been implemented to enhance the performance of $\mathrm{Ti}^{-} \mathrm{Al}_{2} \mathrm{O}_{3}$ in recent years [31,33-35], for example, and even commercial companies have begun to incorporate the technology. The enhancement of $\mathrm{Ti}_{\mathrm{Al}} \mathrm{Al}_{2} \mathrm{O}_{3}$ ultrafast systems at present incorporates closed cycle Gifford-McMahon or pulse tube coolers to reduce thermal focusing, usually in the pre-amplifier stage of a chirped-pulse-amplification (CPA) system. While the thermal properties of such systems are improved, dramatic improvements have not been demonstrated, partly because sapphire already has a large thermal conductivity at room temperature. State-of-the-art $\mathrm{Ti}: \mathrm{Al}_{2} \mathrm{O}_{3} \mathrm{CPA}$ systems have been limited in average power for quite some time to $<100 \mathrm{~W}$. Orders of magnitude increases in the average power capability of $\mathrm{Ti}_{\mathrm{Al}} \mathrm{Al}_{2} \mathrm{O}_{3}$ however may be achieved through the development of powerful new cryogenic pump lasers, providing kilowatt average power capability. Several current efforts to produce picosecond high average power cryogenic lasers at the $1 \mathrm{~J} /$ pulse level and greater at $\mathrm{kHz}$ repetition rates, discussed later in this section, provide validation to designs capable of producing high average power nanosecond cryogenic frequency-doubled green lasers with high efficiency, to be used as pump sources for high-average-power $\mathrm{CPA} \mathrm{Ti}_{\mathrm{Al}} \mathrm{Al}_{2} \mathrm{O}_{3}$ lasers. At the present time green pump sources are typically limited to $10-30 \mathrm{~Hz}$ repetition rates, although lower peak power systems can operate at $\mathrm{kHz}$ repetition rates as well. To produce $\mathrm{HAPP}$ Ti: $\mathrm{Al}_{2} \mathrm{O}_{3}$ systems with high peak and average powers, however, it is likely that all $\mathrm{Ti}: \mathrm{Al}_{2} \mathrm{O}_{3}$ amplifiers as well as their pump lasers will need to be cryogenically-cooled.

A second application for cryogenic laser technology for ultrafast lasers is to build strong joule-level pumps operating with $\mathrm{kHz}$ repetition rates, to drive optical parametric chirped-pulse-amplification (OPCPA) ultrafast systems, recently described as third-generation femtosecond technology [156]. Parametric amplification has garnered much attention recently because it generates only small amounts of heat and is capable of providing very broad gain bandwidths allowing the production of few-cycle systems. The development of borate based nonlinear crystals such as BBO and LBO, materials with high damage thresholds, has considerably aided the development of OPCPA femtosecond lasers. OPCPA has already produced femtosecond laser performance, and is presently limited by currently available pump sources. The need for better pump sources has been discussed in the literature $[156,157]$, with near universal agreement that high average power pumps with very good beam-quality and pulse durations near $\sim 1$ ps and operating in the near-infrared regions are required. Near-infrared sources 
such as $\mathrm{Yb}$ operating near $1030 \mathrm{~nm}$ are expected to provide fundamental, second, and third harmonic pumping at high average power, and the recent intensive development of $\mathrm{Yb}$ based cryogenic laser sources seem well-positioned to address this need.

In Table 10, we show reported demonstrations of cryogenic $\mathrm{Yb}: Y A G, Y b: Y L F$, and $\mathrm{Yb}: \mathrm{Y}_{2} \mathrm{O}_{3}$ in the time period 2003 through 2015, and the output parameters that were achieved. We list, in order from left to right, the year, the crystal type, the pulse energy $E p$, repetition rate $v_{r}$, average power Pav, the gain bandwith $\Delta \lambda_{C}$ (from Table 6), laser operating wavelength $\lambda_{L}$, the Gaussian transform-limited pulsewidth $\delta t_{T L}$, the seed bandwidth $\Delta \lambda_{S}$, the seed pulsewidth $\delta t_{T L S}$, the amplifier bandwidth $\Delta \lambda_{A}$, the amplifier expected pulsewidth $\delta t_{T L A}$, the measured output pulsewidth $\delta t_{M E A S}$, the peak power $P_{P}$, and the corresponding reference $[30,110,132,155,158-169]$. Other acronyms found in the Table are BC (before-recompression), AC (after-recompression), R (cryogenic regenerative amplifier), SHG (second-harmonic-generation), UC for (not re-compressed), and $\mathrm{E}$ for estimated. We also use $\sigma$ to denote use of the $\sigma$ transition in Yb:YLF. The majority of demonstrations listed in Table 10 involve the use of multi-pass cryogenic Yb:YAG or Yb:YLF amplifiers to boost the peak and average powers of picosecond laser systems, with most systems targeted for use as pumps in OPCPA femtosecond lasers. Another application of interest is the generation of table-top X-ray lasers. We also show in Table 10 the frequency-doubled results, where applicable. Most of the reported cryogenic systems in Table 10 use the CPA technique to stretch seed pulses so as to obtain a higher damage threshold; in a few cases, direct amplification was used to avoid the losses and added complexity of CPA systems.

The Gaussian transform-limited pulsewidth $\delta t_{T L}$ is calculated for cryogenic temperatures near $80 \mathrm{~K}$, and uses the bandwidths taken from Table 6 as well as the operating wavelength of the specific laser system. The bandwidth of the seed laser, $\Delta \lambda_{S}$, is taken from the referenced paper where provided, and used to calculated the transform-limited output pulsewidth $\delta t_{S}$ value supported by that bandwidth. For the cryogenic multi-pass or regenerative amplifier, the amplifier bandwidth $\Delta \lambda_{A}$ measurement reported in the publication is stated, and again the transform-limited pulse duration $\delta t_{A}$ supported by that value is calculated. We then show the reported pulsewidth measurement $\delta t_{M E A S}$, allowing one to ascertain, in the reported stretcher-compressor CPA systems, how closely the obtained pulsewidth after compression is to the transform-limited value. The transform-limited pulsewidth for Yb:YAG at $80 \mathrm{~K}$ is about $1.04 \mathrm{ps}$ (bandwidth of about $1.5 \mathrm{~nm}$ at $80 \mathrm{~K}$ ), a value that has not yet been closely approached. This is a consequence of gain-narrowing that takes place in multi-pass amplifiers, and particularly in regenerative amplifiers where many passes of the gain medium occur. Regenerative amplifiers can have bandwidths $<0.2 \mathrm{~nm}$, so the obtained output pulsewidths tend to be $>$ about 8 ps. Imperfect compression stretches the pulses even further. A number of techniques have been used to reduce the effects of gain-narrowing and to provide a seed laser for the demonstrations listed in Table 10 with broadband pulses entering the regenerative amplifier. In order to obtain pulsewidths in the femtosecond regime, researchers have sought laser materials with a broader bandwidth at $80 \mathrm{~K}$. One such material is $\mathrm{Yb}: \mathrm{YLF}$, which has a gain bandwidth of about $27 \mathrm{~nm}$ and is capable of producing $\sim 100 \mathrm{fs}$ pulses. Table 10 shows that sub-ps pulses have been produced by two groups $[155,167]$. Other materials such as Yb:GSAG [30] have been developed as well to drive obtained pulsewidths from $\mathrm{Yb}$ based lasers into the femtosecond regime. $\mathrm{Yb}: \mathrm{CaF}_{2}$ has also been recently developed as a promising fs laser materials, due to a broad bandwidth that remains broad even at $80 \mathrm{~K}$. At room temperature, a $\mathrm{Yb}: \mathrm{CaF}_{2}$ laser is reported with a pulsewidth of $99 \mathrm{fs}$ [111]. A cryogenic laser using the closely related material $\mathrm{Yb}: \mathrm{CaNaF}_{2}$ laser has produced 173 fs pulses [170]. While we have not discussed a number of promising $\mathrm{Yb}$ based laser materials in this paper, choosing to concentrate here only on significant high average as well as peak power results at cryogenic temperatures, it is clear that $\mathrm{Yb}$ lasers are an active and vibrant research topic today, and that in the near future other newer laser materials will be used to advance the state-of-the-art. 
Table 10. Reported Yb:YAG, Yb:YLF, and Yb: $\mathrm{Y}_{2} \mathrm{O}_{3}$ Cryogenic Picosecond and Femtosecond Demonstrations From $2003-2015$.

\begin{tabular}{|c|c|c|c|c|c|c|c|c|c|c|c|c|c|c|}
\hline Year & $\begin{array}{l}\text { Crystal } \\
\text { Type }\end{array}$ & $E_{\mathrm{p}}(\mathrm{mJ})$ & $v_{\mathrm{r}}(\mathrm{Hz})$ & $\operatorname{Pav}(\mathrm{W})$ & $\begin{array}{c}\Delta \lambda_{C}(\mathrm{~nm}) \\
\text { Table } 6\end{array}$ & $\lambda_{L}(\mathrm{~nm})$ & $\delta t_{T L}(p s)$ & $\Delta \lambda_{S}(\mathrm{~nm})$ & $\begin{array}{c}\delta t_{T L S} \\
(\mathrm{ps})\end{array}$ & $\begin{array}{l}\Delta \lambda_{A} \\
(\mathrm{~nm})\end{array}$ & $\begin{array}{c}\delta t_{\text {TLA }} \\
(\mathrm{ps})\end{array}$ & $\begin{array}{l}\delta t_{\text {MEAS }} \\
\text { (ps) }\end{array}$ & $P_{P}(\mathrm{~W})$ & Ref \\
\hline 2003 & $\begin{array}{c}\text { Yb:YLF } \\
\sigma\end{array}$ & $\begin{array}{l}30 \times 10^{-3} \mathrm{BC} \\
18 \times 10^{-3} \mathrm{AC}\end{array}$ & 20 & 3.6 & 27.0 & 1017 & 0.097 & 15 & 0.101 & 5 & 0.304 & 0.795 & $11.64 \times 10^{9}$ & [155] \\
\hline 2007 & Yb:YAG & $0.296 \times 10^{-3}$ & $80 \times 10^{2}$ & 24.0 & 1.5 & 1029 & 1.038 & 1.45 & 10.7 & 1.33 & 11.7 & 11.7 & $25.6 \times 10^{6}$ & [158] \\
\hline 2007 & Yb:YAG & $0.99 \times 10^{-3}$ & $20 \times 10^{3}$ & 19.8 & 1.5 & 1029 & 1.038 & 1.45 & 10.7 & 1.33 & 11.7 & 11.7 & $84.6 \times 10^{6}$ & [158] \\
\hline 2007 & $\begin{array}{l}\text { Yb:YAG } \\
\text { SHG }\end{array}$ & $\begin{array}{c}8 \times 10^{-3} \mathrm{BC} \\
7.5 \times 10^{-3} \mathrm{AC} \\
1.9 \times 10^{-3}\end{array}$ & $10 \times 10^{3}$ & $\begin{array}{l}0.075 \\
0.019\end{array}$ & 1.5 & $\begin{array}{c}1029 \\
515\end{array}$ & 1.038 & 17.0 & 0.092 & 0.5 & 3.1 & $\begin{array}{l}34.5 \\
25.0\end{array}$ & $217.5 \times 10^{6}$ & [159] \\
\hline 2008 & Yb:YAG & $3.7 \times 10^{-6}$ & $78 \times 10^{6}$ & 287 & 1.5 & 1029 & 1.038 & 0.4 & 3.89 & 0.3 & 5.2 & 5.5 & $0.67 \times 10^{6}$ & [160] \\
\hline 2009 & $\begin{array}{c}\text { Yb:YAG } \\
\text { SHG }\end{array}$ & $\begin{array}{l}3.1 \times 10^{-6} \\
1.8 \times 10^{-6}\end{array}$ & $78 \times 10^{6}$ & $\begin{array}{l}240 \\
130\end{array}$ & 1.5 & $\begin{array}{c}1029 \\
515\end{array}$ & 1.038 & 0.4 & 3.89 & $\begin{array}{l}0.3 \\
0.2\end{array}$ & $\begin{array}{l}5.2 \\
1.9\end{array}$ & $\begin{array}{l}8.0 \\
6.4\end{array}$ & $\begin{array}{l}0.39 \times 10^{6} \\
0.26 \times 10^{6}\end{array}$ & [161] \\
\hline 2009 & Yb:YAG & $\begin{array}{l}1.45 \mathrm{BC} \\
1.02 \mathrm{AC} \\
\end{array}$ & 10 & 10.2 & 1.5 & 1029 & 1.038 & - & - & - & - & 8.5 & $0.12 \times 10^{12}$ & [162] \\
\hline 2010 & Yb:YAG & $\begin{array}{l}40 \times 10^{-3} \mathrm{BC} \\
32 \times 10^{-6} \mathrm{AC}\end{array}$ & $2 \times 10^{3}$ & 80 & 1.5 & 1029 & 1.038 & 0.24 & 6.49 & 0.10 & 15.0 & - & $2.13 \times 10^{9}$ & [163] \\
\hline 2010 & $\begin{array}{c}\text { Yb:YAG } \\
\text { SHG }\end{array}$ & $\begin{array}{c}15.16 \times 10^{-6} \\
1.20 \times 10^{-6}\end{array}$ & $50 \times 10^{6}$ & $\begin{array}{c}758 \\
60\end{array}$ & 1.5 & $\begin{array}{c}1029 \\
515\end{array}$ & 1.038 & 0.82 & 1.90 & 0.16 & 9.7 & $\begin{array}{l}12.4 \\
11.8\end{array}$ & $\begin{array}{l}1.23 \times 10^{6} \\
0.10 \times 10^{6}\end{array}$ & [132] \\
\hline 2011 & $\begin{array}{c}\mathrm{Yb}: \mathrm{YAG} \\
\text { SHG }\end{array}$ & $\begin{array}{l}8.38 \times 10^{-6} \\
4.02 \times 10^{-6}\end{array}$ & $50 \times 10^{6}$ & $\begin{array}{l}419 \\
201\end{array}$ & 1.5 & $\begin{array}{c}1029 \\
515\end{array}$ & 1.038 & 0.82 & 1.90 & 0.16 & 9.7 & $\begin{array}{l}12.4 \\
12.4\end{array}$ & $\begin{array}{l}0.68 \times 10^{6} \\
0.32 \times 10^{6}\end{array}$ & [164] \\
\hline 2011 & Yb:YAG & $\begin{array}{l}20 \times 10^{-3} \mathrm{BC} \\
13 \times 10^{-3} \mathrm{AC}\end{array}$ & $2 \times 10^{3}$ & 20 & 1.5 & 1029 & 1.038 & 0.70 & 2.22 & 0.24 & 6.5 & 14.2 & $0.915 \times 10^{9}$ & [165] \\
\hline 2011 & Yb:YAG & $\begin{array}{l}140 \times 10^{-3} \mathrm{BC} \\
100 \times 10^{-3} \mathrm{AC}\end{array}$ & 100 & 10 & 1.5 & 1029 & 1.038 & 0.55 & 2.83 & 0.35 & 4.45 & 4.8 & $20.83 \times 10^{9}$ & [166] \\
\hline 2011 & Yb:YAG & $23 \times 10^{-3} \mathrm{UC}$ & $5 \times 10^{3}$ & 115 & 1.5 & 1030 & 1.040 & 1.0 & 1.56 & 0.30 & 5.2 & - & $4.4 \times 10^{9}(\mathrm{E})$ & [30] \\
\hline 2011 & $\begin{array}{l}\text { Yb:YAG+ } \\
\text { Yb:GSAG }\end{array}$ & $\begin{array}{c}14.6 \times 10^{-3} \mathrm{BC} \\
12 \times 10^{-3} \mathrm{AC}\end{array}$ & $5 \times 10^{3}$ & 60 & - & 1030 & - & 1.1 & 1.42 & 0.70 & 2.2 & 1.6 & $7.5 \times 10^{9}$ & [30] \\
\hline 2012 & $\begin{array}{c}\text { Yb:YLF } \\
\sigma\end{array}$ & $10.6 \times 10^{-3}$ & $10 \times 10^{3}$ & 106 & 27.0 & 1020 & 1.038 & 2.40 & 0.65 & 2.20 & 0.71 & 0.865 & $12.3 \times 10^{9}$ & [167] \\
\hline 2014 & $\begin{array}{c}\mathrm{Yb}: \mathrm{Y}_{2} \mathrm{O}_{3} \\
(\mathrm{R})\end{array}$ & $\begin{array}{c}0.70 \times 10^{-3} \\
(\mathrm{UC})\end{array}$ & $1 \times 10^{3}$ & 0.70 & 14.3 & 1031 & 0.109 & - & - & - & - & - & - & [168] \\
\hline $\begin{array}{l}2015 \\
2015\end{array}$ & $\begin{array}{l}\text { Yb:YAG } \\
\text { Yb:YAG }\end{array}$ & $\begin{array}{c}100 \times 10^{-3}(\mathrm{UC}) \\
72\end{array}$ & $\begin{array}{c}250 \\
1\end{array}$ & $\begin{array}{l}25.0 \\
72\end{array}$ & $\begin{array}{l}1.5 \\
1.5\end{array}$ & $\begin{array}{c}1029 \\
1030.5\end{array}$ & $\begin{array}{l}1.038 \\
1.038\end{array}$ & $\begin{array}{c}- \\
1.0\end{array}$ & $\begin{array}{c}- \\
1.57\end{array}$ & $\begin{array}{l}- \\
0.35\end{array}$ & $\begin{array}{c}- \\
4.45\end{array}$ & $\begin{array}{c}- \\
5.9\end{array}$ & $\begin{array}{c}- \\
8.0 \times 10^{9}\end{array}$ & $\begin{array}{l}{[169]} \\
{[110]}\end{array}$ \\
\hline
\end{tabular}


Figure 47 shows a plot of the energy/pulse and repetition rate as a function of laser average power, using the values found in Table 10, for both fundamental and second-harmonic wavelengths. The energy/pulse varies from typically microjoules at the higher repetition rates $(\mathrm{MHz})$ to large energy/pulse for low repetition rates. The largest energy/pulse obtained is about $1.02 \mathrm{~J}$ at a $10 \mathrm{~Hz}$ repetition rate [162]. For intermediate repetition rates in the $\mathrm{kHz}$ regime, some $\mathrm{mJ}$ of energy/pulse are obtained. The average power associated with low repetition rates and large energy/pulse tend to have average powers $<20 \mathrm{~W}$, while $\mathrm{kHz}$ repetition rates and intermediate $\mathrm{mJ}$ energies tend to be less than $110 \mathrm{~W}$. The highest average powers tend to be associated with $\mathrm{MHz}$ repetition rates and lower $(\mu \mathrm{J})$ energies per pulse. The highest average power, $758 \mathrm{~W}$, was obtained by our group, producing $15.2 \mu \mathrm{J} /$ pulse at a $50 \mathrm{MHz}$ repetition rate [132]. The highest green ps average power obtained at cryogenic temperatures was also demonstrated by our group [164].

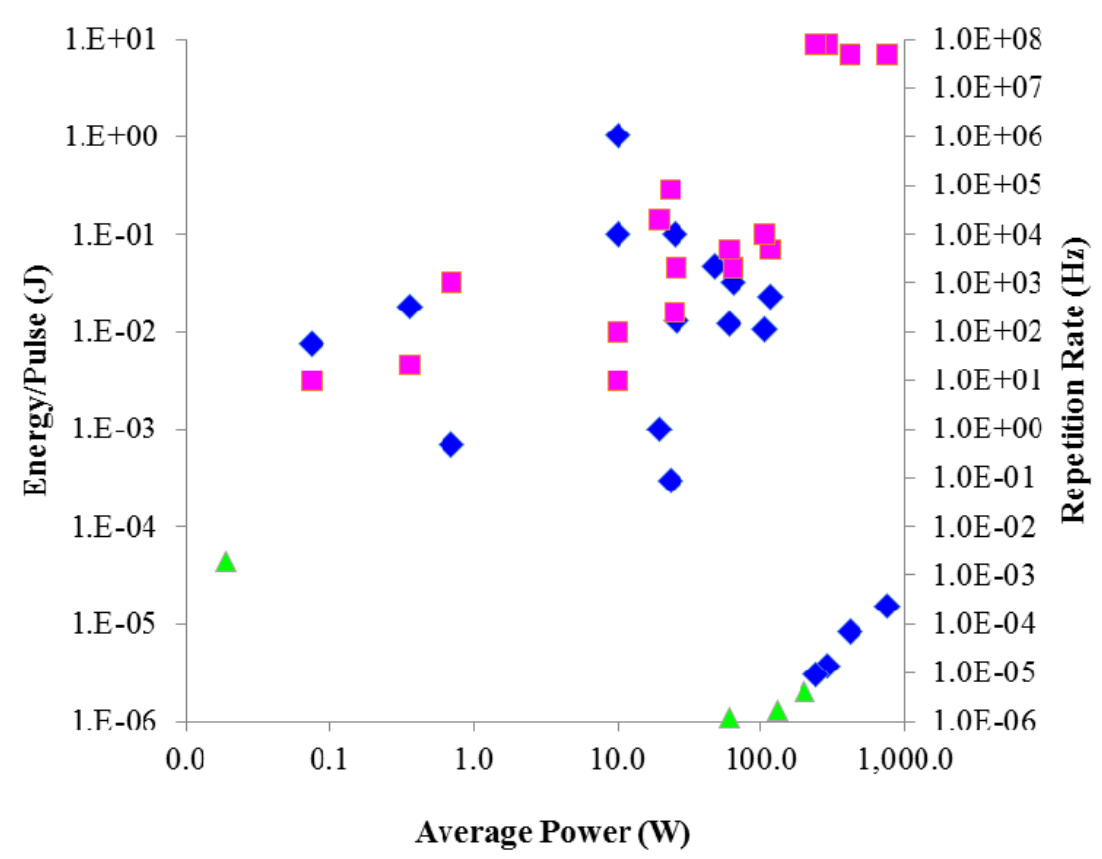

Figure 47. $\mathrm{Yb}: \mathrm{YAG}, \mathrm{Yb}: \mathrm{YLF}, \mathrm{Yb}: \mathrm{Y}_{2} \mathrm{O}_{3}$, and $\mathrm{Yb}: \mathrm{YAG}+\mathrm{Yb}: G S A G$ compressed or direct energy / pulse at fundamental (blue) and second-harmonic (green) wavelengths, and repetition rate (pink), as a function of average power. Numerical values and references are taken from Table 10.

Figure 48 shows the peak power obtained as a function of average power, again using data from Table 10. Fundamental and second-harmonic values are displayed. The highest peak powers are produced by YbYLF based devices and the lowest by high repetition rate, low energy/pulse lasers.

Finally, we mention that there are a number of higher energy /pulse high average power cryogenic laser demonstrations that are currently being developed. One group [139] at DESY has as its goal a $1 \mathrm{~J} /$ pulse, $1 \mathrm{kHz}, 1 \mathrm{~kW}$, few ps Yb:YAG cryogenic laser device that uses innovative an ASE suppressing thin disk, and utilizes 12-pass cryogenic amplifiers. This laser also employs the technique of imaging and vacuum spatial filtering to manage nonlinear effects and produce clean beam profiles, techniques that were pioneered is the earliest high-peak-power laser systems developed in connection with inertial confinement fusion [37]. We also mention the work of HiLASE [171,172], where large aperture high energy/pulse Yb:YAG cryogenically He gas cooled lasers are being developed. In [152] a $10 \mathrm{~J} /$ pulse, $10 \mathrm{~Hz}$ cryogenic amplifier is already developed, and a $100 \mathrm{~J} /$ pulse cryogenic amplifier is expected to be demonstrated in 2015, while in [173] a $1 \mathrm{~J}, 1$ ps at $120 \mathrm{~Hz}$ system in development as part of the HiLASE program. 


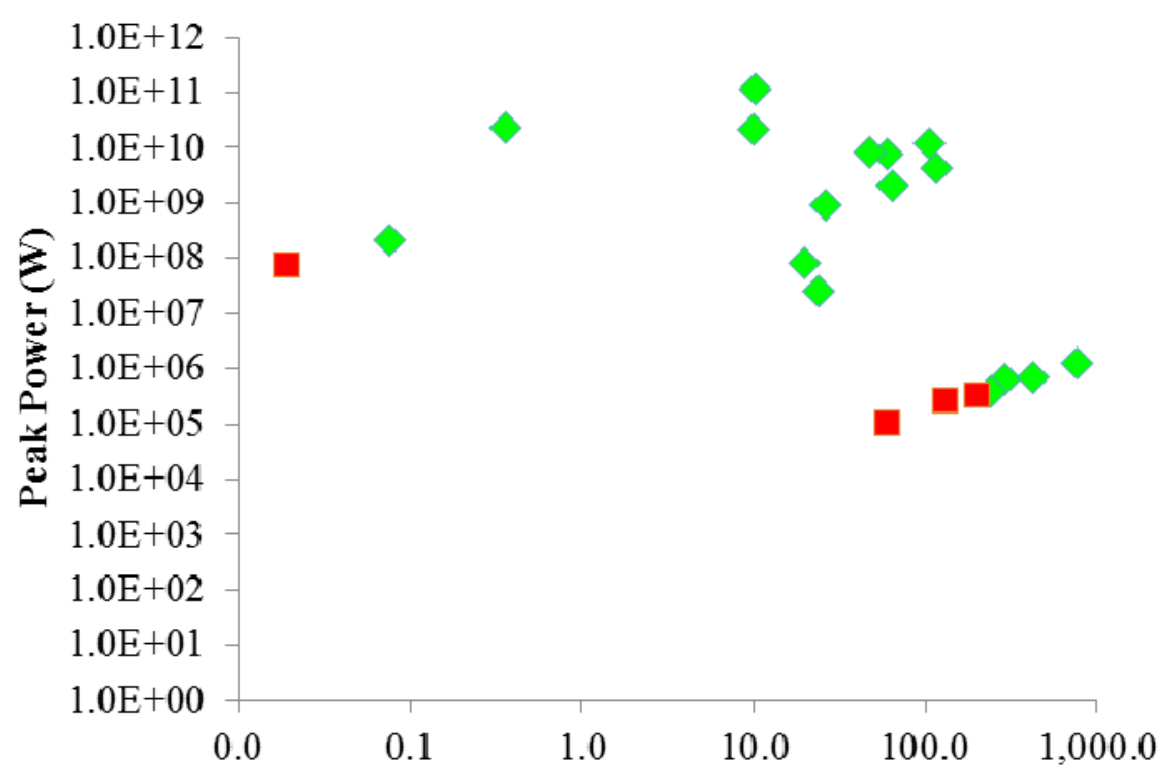

Average Power (W)

Figure 48. $\mathrm{Yb}: Y A G, Y b: Y L F$, and $Y b: Y A G+Y b: G S A G$ compressed or direct peak power for fundamental (green) and second-harmonic (red) wavelengths, as a function of average power. Numerical values and references are taken from Table 10.

\section{Conclusions}

In this paper, we have endeavored to summarize the history of cryogenic lasers, review the most important spectral, thermal, and thermo-optic properties, provide a summary of the measured parameters at cryogenic temperatures, and to provide a relatively up-to-date snapshot of the development of CW, nanosecond, picosecond, and femtosecond cryogenic laser development. Cryogenic laser development has accelerated in recent years, and we believe we have only just begun to understand all of the benefits the technology brings to the development of advanced sophisticated laser devices that address a large swath of scientific, military, and commercial applications. In various sections of this paper we have pointed out the critical need for measurements of key parameters, such as the nonlinear indices and damage thresholds at cryogenic temperatures, that can have a profound impact on the scaling of cryogenic laser devices. It is our hope that the cryogenic laser community will respond to this need in the near future. The development of HAPP lasers that have high peak and average powers while maintaining near-diffraction-limited beam characteristics, independent of average power, is now well-underway, and the promise, progress, and prospects of cryogenic lasers are very bright indeed.

Acknowledgments: The research discussed here was partially funded by the USA. National Science Foundation, under Grant \# DMR-1410727.

Author Contributions: David C. Brown conceived of and wrote the manuscript, performed the numerical calculations, and designed the liquid nitrogen cryostat used for the reported absorption measurements. Sten Tornegård provided helpful guidance and discussions in preparing the manuscript as well as important references. Joseph Kolis, Colin McMillen, Cheryl Moore, Liurukawa Sanjeewa, and Christopher Hancock supervised growing the hydrothermal crystals, polished them, obtained the absorption spectroscopy, and built and tested the liquid nitrogen cryostat. Brown dedicates this paper to the memory of his very good friend, FarresP.Mattar, a brilliant polymath and wonderful human being who always understood the meaning of le joie de vivre.

Conflicts of Interest: The authors declare no conflict of interest. 


\section{References}

1. Lacovara, P.; Choi, H.K.; Wang, C.A.; Aggarwal, R.L.; Fan, T.Y. Room-temperature diode-pumped Yb:YAG laser. Opt. Lett. 1991, 16, 1089-1091. [CrossRef] [PubMed]

2. Brown, D.C. The promise of cryogenic lasers. IEEE J. Sel. Top. Quantum Electron. 2005, 11, 587-599. [CrossRef]

3. Fan, T.Y.; Ripin, D.J.; Aggarwal, R.L.; Ochoa, J.R.; Chann, B.; Tilleman, M.; Spitzberg, J. Cryogenic Yb ${ }^{3+}$-doped solid-state lasers, Invited Paper. IEEE J. Sel. Top. Quantum Electron. 2007, 13, 448-459. [CrossRef]

4. Wall, K.F.; Moulton, P.F. High power operation of cryogenic Yb:YAG. In Proceedings of the SPIE, San Jose, CA, USA, 23 January 2007.

5. Keyes, R.J.; Quist, T.M. Injection luminescent pumping of $\mathrm{CaF}_{2}: \mathrm{U}^{3+}$ with GaAs diode lasers. Appl. Phys. Lett. 1974, 4, 50-52. [CrossRef]

6. Moulton, P.F. Spectroscopic and laser characteristics of $\mathrm{Ti}^{-} \mathrm{Al}_{2} \mathrm{O}_{3}$. J. Opt. Soc. Am. B 1986, 3, $125-133$. [CrossRef]

7. Moulton, P.F. Efficient cw optically pumped Ni:MgF 2 laser. Opt. Lett. 1978, 3, 164-166. [CrossRef] [PubMed]

8. Moulton, P.F. Broadly tunable cw operation of $\mathrm{Ni}_{\mathrm{MgF}}$ and $\mathrm{Co}: \mathrm{MgF}_{2}$ lasers. Appl. Phys. Lett. 1979, 35, 838-840. [CrossRef]

9. Moulton, P.F. Pulse-pumped operation of divalent transition-metal lasers. IEEE J. Quantum Electron. 1982, 18, 1185-1188. [CrossRef]

10. Moulton, P.F. An investigation of the Co: $\mathrm{MgF}_{2}$ system. IEEE J. Quantum Electron. 1985, 21, $1582-1595$. [CrossRef]

11. Schulz, P.A. Liquid-nitrogen-cooled Ti: $\mathrm{Al}_{2} \mathrm{O}_{3}$ laser. IEEE J. Quantum Electron. 1991, 27, 1039-1047. [CrossRef]

12. Johnson, J.F.; Dietz, R.E.; Guggenheim, H.J. Optical maser oscillation from $\mathrm{Ni}^{2+}$ in $\mathrm{MgF}_{2}$ involving simultaneous emission of phonons. Phys. Rev. Lett. 1963, 11, 318-320. [CrossRef]

13. Doehler, J.; Solin, S.A. A compact efficient liquid nitrogen system for cooling solid state lasers: Application to YAG:Nd ${ }^{3+}$ and ABC-YAG. Rev. Sci. Instr. 1972, 43, 1189-1193. [CrossRef]

14. Beck, R.; Gürs, K. Ho laser with 50-W output and 6.5\% slope efficiency. J. Appl. Phys. 1975, 48, 5224-5225. [CrossRef]

15. Kaminskii, A.A. Laser Crystals; Springer-Verlag: Berlin, Germany; Heidelberg, Germany; New York, NY, USA, 1990.

16. Kaminskii, A.A. Crystalline Lasers: Physical Processes and Operating Schemes; CRC Press: Boca Raton, FL, USA, 1996.

17. Bowess, C. Liquid Nitrogen Cooled Ruby Laser. US Patent 3339150, 29 August 1967.

18. McMahon, D.H. Cooling System for Laser Media. US Patent 3676798, 11 July 1972.

19. Brown, D.C.; Nelson, R. Ultra-high-average-power diode-pumped Nd:YAG and Yb:YAG lasers. BMDO Phase I Small Business Innovation Research Contract \# N00P14-97-P-2064 Final Report. 1998.

20. Brown, D.C. Ultrahigh-average-power diode-pumped Nd:YAG and Yb:YAG lasers. IEEE J. Quantum Electron. 1997, 33, 863-873. [CrossRef]

21. Brown, D.C. Nonlinear Thermal Distortion in YAG Rod Amplifiers. IEEE J. Quantum Electron. 1998, 33, 2383-2392. [CrossRef]

22. Brown, D.C. Nonlinear Thermal and Stress Effects and Scaling Behavior of YAG Slab Amplifiers. IEEE J. Quantum Electron. 1998, 33, 2393-2402. [CrossRef]

23. Fan, T.Y.; Daneu, J.J. Thermal coefficients of the optical path length and refractive index in YAG. Appl. Opt. 1998, 37, 1735-1637. [CrossRef]

24. Fan, T.Y.; Crow, T.; Hoden, B. Cooled Yb:YAG for high power solid-state lasers. In Airborne Laser Advanced Technology (Proceedings of SPIE); SPIE Press: Bellingham, MA, USA, 1998; Volume 3381, pp. 200-215.

25. Glur, H.; Lavi, R.; Grat, T. Reductions of thermally induced lenses in Nd; YAG with low temperatures. IEEE J. Quantum Electron. 2004, 40, 499-503. [CrossRef]

26. Wynne, R.; Daneu, J.J.; Fan, T.Y. Thermal coefficients of the expansion and refractive index in YAG. Appl. Opt. 1999, 38, 3282-3284. [CrossRef] [PubMed]

27. Brown, D.C.; Cone, R.L.; Sun, Y.; Equall, R.W. Yb:YAG absorption at ambient and cryogenic temperatures. IEEE J. Sel. Top. Quantum Electron. 2005, 11, 604-612. [CrossRef]

28. Brown, D.C.; Bruno, T.M.; Vitali, V. Saturated Absorption Effects in CW-Pumped Solid-State Lasers. IEEE J. Quantum Electron. 2010, 40, 1717-1725. [CrossRef] 
29. Aggarwal, R.L.; Ripin, D.J.; Ochoa, J.R.; Fan, T.Y. Measurement of thermo-optic properties of $\mathrm{Y}_{3} \mathrm{Al}_{5} \mathrm{O}_{12}$, $\mathrm{Lu}_{3} \mathrm{Al}_{5} \mathrm{O}_{12}, \mathrm{YAlO}_{3}, \mathrm{LiYF}_{4}, \mathrm{BaY}_{2} \mathrm{~F}_{8}, \mathrm{KGd}\left(\mathrm{WO}_{4}\right)_{2}$, and $\mathrm{KY}\left(\mathrm{WO}_{4}\right)_{2}$ laser crystals in the 80-300 $\mathrm{K}$ temperature range. J. Appl. Phys. 2005, 98, 1-14.

30. Rand, D.; Miller, D.; Ripin, D.J.; Fan, T.Y. Cryogenic $\mathrm{Yb}^{3+}$-doped materials for pulsed solid-state laser applications. Opt. Mater. Express 2011, 1, 434-450. [CrossRef]

31. Backus, S.; Durfee, C.G., III; Mourou, G.; Kapteyn, H.; Murnane, M.M. 0.2-TW laser system at 1 Hz. Opt. Lett. 1997, 22, 1256-1258. [CrossRef] [PubMed]

32. Backus, S.; Durfee, C.G., III; Murnane, M.M.; Kapteyn, H. High power ultrafast lasers. Rev. Sci. Instrum. 1998, 69, 1207-1223. [CrossRef]

33. Matsushima, I.; Yashiro, H.; Tomie, T. A 37\% efficiency, kilohertz repetition rate cryogenically cooled Ti:Sapphire regenerative amplifier. Jpn. J. Appl. Phys. 2005, 25, L823-L826. [CrossRef]

34. Dantan, A.; Laurat, J.; Ourjoumtsev, A.; Tualle-Brouri, R.; Grangier, P. Femtosecond Ti:Sapphire cryogenic amplifier with high gain and MHz repetition rate. Opt. Express 2007, 15, 8864-8870. [CrossRef] [PubMed]

35. Planchon, T.A.; Amir, W.; Childress, C.; Squier, J.A.; Durfee, C.G. Measurement of pump-induced transient lensing in a cryogenically-cooled high average power Ti:Sapphire amplifier. Opt. Express 2008, 16, 18557-18564. [CrossRef] [PubMed]

36. Maiman, T.H. Stimulated Optical Radiation in Ruby. Nature 1960, 187, 493-494. [CrossRef]

37. Brown, D.C. High-Peak-Power Nd:Glass Laser Systems. In Springer Series in Optical Sciences; Springer-Verlag: Berlin, Germany; Heidelberg, Germany, 1981.

38. Brown, D.C.; Vitali, V.A. Yb:YAG kinetics model including saturation and power conservation. IEEE J. Quantum Electron. 2011, 47, 3-12. [CrossRef]

39. Kittel, C. Introduction to Solid-State Physics, 8th ed.; John Wiley and Sons, Inc.: Hoboken, NJ, USA, 2005.

40. Zhou, Y.; Xiang, H.; Feng, Z. Theoretical investigation on mechanical and thermal properties of a promising thermal barrier material: $\mathrm{Yb}_{3} \mathrm{Al}_{5} \mathrm{O}_{12}$. J. Mater. Sci. Technol. 2014, 30, 631-638. [CrossRef]

41. Jiji, L.M. Heat Conduction, 3rd ed.; Springer-Verlag: Berlin, Germany; Heidelberg, Germany; New York, NY, USA, 2009.

42. Klemens, P.G. Thermal resistance due to point defects at high temperature. Phys. Rev. 1960, 119, 507-509. [CrossRef]

43. McMillen, C.D.; Thompson, D.; Tritt, T.; Kolis, J.W. Hydrothermal single crystal growth of $\mathrm{Lu}_{2} \mathrm{O}_{3}$ and lanthanide doped $\mathrm{Lu}_{2} \mathrm{O}_{3}$. Cryst. Growth Des. 2011, 11, 4386-4391. [CrossRef]

44. Greibner, U.; Petrov, V.; Petermann, K.; Peters, V. Passively mode-locked $\mathrm{Yb}: \mathrm{Lu}_{2} \mathrm{O}_{3}$ laser. Opt. Express 2004, 12, 3125-3130. [CrossRef]

45. Slack, G.A.; Oliver, D.W. Thermal conductivity of garnets and phonon scattering by rare-earth ions. Phys. Rev. 1971, 4, 592-609. [CrossRef]

46. Holland, M.G. Thermal conductivity of several optical maser materials. J. Appl. Phys. 1962, 33, $2910-2911$. [CrossRef]

47. Padture, N.P.; Klemens, P.G. Low thermal conductivity in garnets. J. Am. Ceram. Soc. 1997, 80, 1018-1020. [CrossRef]

48. Klopp, P. New $\mathrm{Yb}^{3+}$-Doped Laser Materials and Their Application in Continuous-Wave and Mode-Locked Lasers. Ph.D. Thesis, University of Berlin, Berlin, Germany, 2006.

49. Fredrich-Thornton, S.T. Nonlinear Losses in Single Crystalline and Ceramic Yb:YAG Thin Disk Lasers. Ph.D. Thesis, University of Hamburg, Hamburg, Germany, 2010.

50. Beil, K.; Saraceno, C.J.; Schriber, C.; Emaury, F.; Heckl, O.H.; Baer, C.R.E.; Golling, M.; Südmeyer, T.; Keller, U.; Kränkel, C.; et al. Yb-doped mixed sesquioxides for ultrashort pulse generation in the thin disk laser setup. Appl. Phys. B Lasers Opt. 2013, 113, 13-18. [CrossRef]

51. Koopman, P.; Lamrini, S.; Scholle, K.; Fuhrberg, P.; Petermann, K.; Huber, G. High Power Diode Pumped 1 $\mu \mathrm{m}$ laser operation of Tm: $\mathrm{Lu}_{2} \mathrm{O}_{3}$. In Proceedings of the CLEO/QELS Conference 2010, Optical Society of America Paper CMDD1, San Jose, CA, USA, 16-21 May 2010.

52. Nikogosyan, D.N. Properties of Optical and Laser-Related Materials: A Handbook; John Wiley and Sons, Inc.: Hoboken, NJ, USA, 1998. 
53. Garrec, B.L.; Cardinali, V.; Bourdet, G. Thermo-optical measurements of ytterbium doped ceramics $\left(\mathrm{Sc}_{2} \mathrm{O}_{3}\right.$, $\left.\mathrm{Y}_{2} \mathrm{O}_{3}, \mathrm{Lu}_{2} \mathrm{O}_{3}, \mathrm{YAG}\right)$ and crystals (YAG, $\mathrm{CaF}_{2}$ ) at cryogenic temperatures. In High-Power, High-Energy, and High-Intensity Laser Technology; and Research Using Extreme Light; Entering New Frontiers with Petawatt-Class Lasers Proceedings of SPIE; Hein, J., Korn, G., Silva, L.O., Eds.; SPIE Press: Bellingham, WA, USA, 2013. [CrossRef]

54. Popov, P.A.; Federov, P.P.; Kuznetsov, V.A.; Konyushkin, V.A.; Osiko, V.V.; Basiev, T.T. Thermal conductivity of single crystals of $\mathrm{Ca}_{1-x} \mathrm{Yb}_{x} \mathrm{~F}_{2+x}$ solid solutions. Dokl. Phys. 2008, 53, 198-200. [CrossRef]

55. Toulokian, Y.S.; Kirby, R.K.; Taylor, R.E.; Lee, T.Y.R. Thermophysical Properties of Matter; Plenum Press: New York, NY, USA, and London, UK, 1977.

56. Linde, D.R., Ed.; Handbook of Chemistry and Physics, 76th ed.; CRC Press: Boca Raton, FL, USA, 1995.

57. Powell, R.L.; Blanpied, W.A. Thermal conductivity of metals and alloys at low temperature. In National Bureau of Standards Circular 556; U.S. Government Printing Office: Washington, D.C., USA, 1954.

58. Yasuhara, R.; Furuse, H.; Iwamoto, A.; Kawanaka, J.; Yagatitani, T. Evaluation of thermo-optic characteristics of cryogenically cooled Yb:YAG ceramics. Opt. Express 2012, 20, 29532-29539. [CrossRef] [PubMed]

59. Cardinalli, V.; Marmois, E.; Garrec, B.L.; Bourdet, G. Determination of the thermo-optic coefficient $\mathrm{d} n / \mathrm{d} T$ of ytterbium doped ceramics $\left(\mathrm{Sc}_{2} \mathrm{O}_{3}, \mathrm{Y}_{2} \mathrm{O}_{3}, \mathrm{Lu}_{2} \mathrm{O}_{3}, \mathrm{YAG}\right)$ and crystals $\left(\mathrm{YAG}, \mathrm{CaF}_{2}\right)$ and neodymium doped phosphate glass at cryogenic temperature. Opt. Mater. 2012, 34, 990-994. [CrossRef]

60. Reeber, R.R.; Wang, K. Lattice parameters and thermal expansion of important semiconductors and their substrates. Mater. Res. Soc. Symp. 2000, 622, T635.1-T635.6. [CrossRef]

61. Senyshyn, A.; Vasylechko, L. Low temperature crystal structure behavior of complex yttrium aluminum oxides $\mathrm{YAlO}_{3}$ and $\mathrm{Y}_{3} \mathrm{Al}_{5} \mathrm{O}_{12}$. Acta Phys. Pol. A 2013, 124, 329-335. [CrossRef]

62. Munro, R.G. Analytical representations of elastic moduli data with simultaneous dependence on temperature and porosity. J. Res. Natl. Inst. Stand. Technol. 2004, 109, 497-503. [CrossRef]

63. Krupke, W.F.; Shinn, M.D.; Marion, J.E.; Caird, J.A.; Stokowski, S.E. Spectroscopic, optical, and thermomechnical properties of neodymium- and chromium-doped gadolinium scandium gallium garnet. J. Opt. Soc. Am. B 1986, 3, 102-114. [CrossRef]

64. Boyd, R.W.; Lukishova, S.G. Topics in Applied Physics. In Self-Focusing: Past and Present; Springer-Verlag: New York, NY, USA, 2009.

65. Soures, J.M. Selected Papers on High Power Lasers; SPIE Press: Bellingham, MA, USA, 1991.

66. Strickland, D.; Mourou, G. Compression of amplified chirped optical pulses. Opt. Commun. 1985, 56, $219-221$. [CrossRef]

67. Boyd, R.W. Nonlinear Optics, 2nd ed.; Academic Press: London, UK; San Diego, CA, USA, 2003.

68. DeSalvo, R.; Said, A.A.; Hagan, D.J.; van Stryland, E.W.; Sheik-Bahae, M. Infrared to ultraviolet measurements of two-photon absorption and $\mathrm{n}_{2}$ in wide bandgap solids. IEE J. Quantum Electron. 1996, 32, 1324-1333. [CrossRef]

69. Major, A.; Yoshino, F.; Nikolakakos, I.; Aitchison, J.S.; Smith, P.W.E. Dispersion of the nonlinear index in sapphire. Opt. Lett. 2004, 29, 602-604. [CrossRef] [PubMed]

70. Diels, J.C. Ultrashort Laser Pulse Phenomena, 2nd ed.; Elsevier, Inc.: Amsterdam, The Netherlands, 2006.

71. Milam, D. Review and assessment of measured values of the nonlinear refractive-index coefficient of fused silica. Appl. Opt. 1998, 37, 546-550. [CrossRef] [PubMed]

72. Senatsky, Y.; Shirahawa, A.; Sato, Y.; Hagiwara, J.; Lu, J.; Ueda, K.; Yagi, H.; Yanagitani, T. Nonlinear refractive index of ceramic laser media and perspectives on their usage in a high-power laser driver. Laser Phys. Lett. 2004, 1, 500-506. [CrossRef]

73. Sanghera, J.; Kim, W.; Villalobos, G.; Shaw, B.; Baker, C.; Frantz, J.; Sadowski, B.; Aggarwal, I. Ceramic Laser Materials. Materials 2012, 5, 258-277. [CrossRef]

74. Milam, D. Nonlinear refractive index of fluoride crystals. Appl. Phys. Lett. 1977, 32, 822-825. [CrossRef]

75. Lagatsky, A.A.; Sarmani, A.R.; Brown, C.T.A.; Sibbett, W.; Kisel, V.E.; Selivanov, A.G.; Denisov, I.A.; Troshlin, A.E.; Yumashev, K.V.; Kuleshov, N.V.; et al. $\mathrm{Yb}^{3+}$-doped $\mathrm{YVO}_{4}$ crystal for efficient kerr-lens mode locking in solid-state lasers. Opt. Lett. 2005, 30, 3234-3236. [CrossRef] [PubMed]

76. Vodchits, A.I.; Orlovich, V.A.; Apanasevich, P.A. Nonlinear refractive index of vanadate crystals in the near IR region. J. Appl. Spectrosc. 2012, 78, 918-921. [CrossRef] 
77. Major, A.; Nikolakakos, I.; Aitchison, J.S.; Ferguson, A.I.; Langford, N.; Smith, P.W.E. Characterization of the nonlinear refractive index of the laser crystal $\mathrm{Yb}: \mathrm{KGd}\left(\mathrm{WO}_{4}\right)_{2}$. Appl. Phys. B Lasers Opt. 2003, 77, 433-436. [CrossRef]

78. Geints, Y.E.; Kabanov, A.M.; Zemlyanov, A.A.; Bykova, E.E.; Bukin, O.A.; Golik, S.S. Kerr-driven nonlinear refractive index of air at 800 and $400 \mathrm{~nm}$ measured through femtosecond laser pulse filimentation. Appl. Phys. Lett. 2011, 99, 1-3. [CrossRef]

79. DeFranzo, A.C.; Pazol, B.G. Index of refraction measurement of sapphire at low temperatures and visible wavelengths. Appl. Opt. 1993, 32, 2224-2234. [CrossRef] [PubMed]

80. Uemura, S.; Torizuka, K. Development of a diode-pumped kerr-lens mode-locked Cr:LiSAF laser. IEEE J. Quantum Electron. 2003, 39, 68-73. [CrossRef]

81. Peterson, R.D.; Pham, A.T.; Jensson, H.P.; Cassanho, A.; Castillo, V. Thermo-optical comparison of LiSAF, LiCAF, and LiSGaF. In Proceedings of the Advanced Solid-State Laser Conference, Boston, MA, USA, 31 January 1999.

82. Payne, S.A.; Smith, L.K.; Beach, R.L.; Chai, B.H.T.; Tassano, J.K.; DeLoach, L.D.; Kway, W.L.; Solarz, R.W.; Krupke, W.F. Properties of Cr:LiSrAlF 6 crystals for laser operation. Appl. Opt. 1994, 33, 5526-5536. [CrossRef] [PubMed]

83. Woods, B.W.; Payne, S.A.; Marion, J.E.; Hughes, R.S.; Davis, L.E. Thermomechanical and thermo-optical properties of the $\mathrm{LiCaAlF}_{6}: \mathrm{Cr}^{3+}$ laser material. J. Opt. Soc. Am. B 1991, 8, 970-977. [CrossRef]

84. Ileri, B. Lattice Matching of Epitaxial Rare-Earth-Doped Dielectric PLD-Films. Ph.D. Thesis, University of Hamburg, Hamburg, Germany, 2007.

85. Weber, M.J. Handbook of Optical Materials: Supplement 2; CRC Press: Boca Raton, FL, USA, 2003.

86. Morrison, C.A.; Leavitt, R.P. Handbook on the Physics and Chemistry of Rare Earths; North-Holland: Amsterdam, The Netherlands, 1982.

87. Barnes, N.P.; Gettemy, D.J. Temperature variation of the refractive indices of yttrium lithium flouride. J. Opt. Soc. Am. 1980, 70, 1244-1247. [CrossRef]

88. Zelmon, D.E.; Lee, J.J.; Currin, K.M.; Northridge, J.M.; Perlov, D. Revisiting the optical properties of Nd doped yttrium orthovanadates. Appl. Opt. 2010, 49, 644-647. [CrossRef] [PubMed]

89. Zelmon, D.E.; Northridge, J.M.; Haynes, N.D.; Perlov, D.; Petermann, K. Temperature-dependent Sellmeier equations for rare-earth sesquioxides. Appl. Opt. 2013, 52, 3824-3828. [CrossRef] [PubMed]

90. Paunescu, G. Ytterbium-Doped Femtosecond Solid-State Lasers. Ph.D. Thesis, Friedrich-Schiller University, Jena, Germany, 2006.

91. Singh, J. Optical Properties of Condensed Matter and Applications; John Wiley and Sons, Ltd.: Hoboken, NJ, USA, 2006.

92. Malitson, I.H. Interspeciman comparison of the refractive index of fused silica. JOSA 1965, 55, 1205-1209. [CrossRef]

93. Koechner, W. Solid-State Laser Engineering, 5th ed.; Springer Series in Optical Sciences; Springer-Verlag: Berlin, Germany; Heidelberg, Germany, 1999.

94. Wood, R.M. Laser-Induced Damage of Optical Materials; Series in Optics and Optoelectronics; Institute of Physics Publishing: Bristol, UK, 2003.

95. Stuart, B.C.; Feit, M.D.; Herman, S.; Rubenchik, A.M.; Shore, B.W.; Perry, M.D. Optical ablation by high-power short-pulse lasers. J. Opt. Soc. Am. B 1996, 13, 459-468. [CrossRef]

96. Wang, H.; Zhang, W.; He, H. Laser-induced damage behaviors of antireflective coatings at cryogenic condition. Appl. Opt. 2012, 51, 8687-8692. [CrossRef] [PubMed]

97. Yang, L.; Yuan, H.D.; Deng, H.X.; Xiang, X.; Zheng, W.G.; He, S.B.; Jiang, Y.; Lv, H.B.; Ye, L.; Wang, H.J.; et al . Influence of ambient temperature on nanosecond and picosecond laser-induced bulk damage of fused silica. Adv. Cond. Matter Phys. 2014, 2014, 1-7. [CrossRef]

98. Mikami, K.; Motokoshi, S.; Fujita, M.; Jitsuno, T.; Kawanaka, J.; Yasuhara, R. Temperature dependence of laser-induced damage threshold in silica glass. J. Phys. Conf. Ser. 2010, 244, 1-7. [CrossRef]

99. Mikami, K.; Motokoshi, S.; Somekawa, T.; Jitsuno, T.; Fujita, M.; Tanake, K.A. Temperature dependence of laser-induced damage threshold of optical coatings at different pulsewidths. Opt. Express 2013, 21, 28719-28728. [CrossRef] [PubMed] 
100. Brown, D.C.; Tornegård, S.; Kolis, J. Cryogenic nanosecond and picosecond high average and peak power (HAPP) pump lasers for ultrafast applications, Paper Submitted to High Power Laser Sci. and Engin. 2015.

101. Eggleston, J.M.; DeShazer, L.G.; Kangas, K.W. Characteristics and kinetics of Ti:Sapphire oscillators. IEEE J. Quantum Electron. 1988, 24, 1009-1015. [CrossRef]

102. Chase, L.L.; Payne, S.A.; Smith, L.K.; Kway, W.L.; Newkirk, H.W.; Chai, B.H.T.; Long, M. Laser performance and spectroscopy of $\mathrm{Cr}^{3+}$ in $\mathrm{LiCaAlF}_{6}$ and $\mathrm{LiSrAlF}_{6}$. In Proceedings of the Advanced Solid-State Lasers Conference, Cape Cod, MA, USA, 1 May 1989.

103. Ricaud, S.; Papadopoulos, D.N.; Camy, P.; Moncorge, R.; Courjaud, A.; Mottay, E.; Georges, P.; Druon, F. Highly efficient, high-power, broadly tunable cryogenically cooled and diode-pumped $\mathrm{Yb}: \mathrm{CaF}_{2}$. Opt. Lett. 2010, 35, 3757-3759. [CrossRef] [PubMed]

104. Brown, D.C.; McMillen, C.D.; Moore, C.; Kolis, J.W.; Envid, V. Spectral properties of hydrothermally-grown $\mathrm{Nd}: \mathrm{LuAG}, \mathrm{Yb}: \mathrm{LuAG}$, and $\mathrm{Yb}: \mathrm{Lu}_{2} \mathrm{O}_{3}$ laser materials. J. Lumin. 2014, 148, 26-32. [CrossRef]

105. Petermann, K.; Huber, G.; Fornasiero, L.; Kuch, S.; Mix, E.; Peters, V.; Basun, S.A. Rare-earth-doped sesquioxides. J. Lumin. 2010, 87-89, 973-975. [CrossRef]

106. Körner, J.; Jambunathan, V.; Hein, J.; Siefert, R.; Loeser, M.; Siebold, M.; Schramm, U.; Sikocinshi, P.; Lucianetti, A.; Mocek, T.; et al. Spectroscopic characterization of $\mathrm{Yb}^{3+}$-doped laser materials at cryogenic temperatures. Appl. Phys. B Lasers Opt. 2014, 116, 75-81. [CrossRef]

107. Peters, V. Growth and Spectroscopy of Ytterbium-Doped Sesquioxides. Ph.D. Thesis, University of Hamburg, Hamburg, Germany, 2001.

108. Zapata, L.; Ripin, D.J.; Fan, T.Y. Power scaling of cryogenic Yb:LiYF 4 lasers. Opt. Lett. 2010, 35, $1854-1856$. [CrossRef] [PubMed]

109. Kawanaka, J.; Yamakawa, K.; Niskioka, H.; Ueda, K. Inproved high-field laser characteristics of a diode-pumped $\mathrm{Yb}: \mathrm{LiYF}_{4}$ crystal at low temperature. Opt. Express 2002, 10, 455-460. [CrossRef] [PubMed]

110. Rand, D.A.; Shaw, S.E.J.; Ochoa, J.R.; Ripin, D.J.; Taylor, A.; Fan, T.Y.; Martin, H.; Hawes, S.; Zhang, J.; Sarkisyan, S.; et al. Picosecond pulses from a cryogenically cooled, composite amplifier using Yb:YAG and Yb:GSAG. Opt. Lett. 2011, 36, 340-342. [CrossRef] [PubMed]

111. Friebel, F.; Druon, F.; Boudeile, J.; Papadopoulus, D.N.; Hanna, M.; Georges, P.; Camy, P.; Doualon, J.L.; Benayad, A.; Moncorgé, R.; et al. Diode-pumped 99 fs Yb:CaF 2 oscillator. Opt. Lett. 2009, 34, 1474-1476. [CrossRef] [PubMed]

112. McMillen, C.; Kolis, J.; Sanjeewa, D.; Moore, C.; Brown, D.C. Hydrothermal growth and spectroscopy of doped $\mathrm{Lu}_{2} \mathrm{O}_{3}$ single crystals. In Paper presented at 20th American Conference on Crystal Growth and Epitaxy, Nonlinear Optical and Host Materials Session 4, Big Sky, MT, USA, 5 August 2015.

113. Heckl, O.H.; Peters, R.; Krankel, C.; Baer, C.R.E.; Saraceno, C.J.; Sudmeyer, T.; Petermann, K.; Keller, U.; Huber, G. Continuous-wave Yb-doped sesquioxide this disk laser with up to $300 \mathrm{~W}$ output and $74 \%$ efficiency. In Ultrafast Lasers I Session, AMD1, Advanced Solid-State Photonics Conference, San Diego, CA, USA, 31 January-3 February 2010.

114. Peters, R.; Krankel, C.; Petermann, K.; Huber, G. Broadly tunable high-power $\mathrm{Yb}: \mathrm{Lu}_{2} \mathrm{O}_{3}$ thin disk laser with 80\% slope efficiency. Opt. Express 2007, 15, 7075-7082. [CrossRef] [PubMed]

115. Mun, J.H.; Jouini, A.; Novoselov, A.; Yoshikawa, A.; Kasamoto, T.; Ohta, H.; Shibata, H.; Isshiki, M.; Waseda, Y.; Boulon, G.; et al. Thermal and optical properties of $\mathrm{Yb}^{3+}$-doped $\mathrm{Y}_{2} \mathrm{O}_{3}$ single crystal grown by the micro-pulling-down method. Jpn. J. Appl. Phys. 2006, 45, 5885-5888. [CrossRef]

116. McMillen, C.D.; Kolis, J.W. Hydrothermal single crystal growth of $\mathrm{Sc}_{2} \mathrm{O}_{3}$ and lanthanide-doped $\mathrm{Sc}_{2} \mathrm{O}_{3}$. J. Cryst. Growth 2008, 310, 1939-1942. [CrossRef]

117. McMillen, C.D.; Kolis, J.W. Bulk single crystal growth from hydrothermal solutions. Philos. Mag. 2012, 92, 2686-2711. [CrossRef]

118. McMillen, C.; Mann, M.; Fan, J.; Zhu, L.; Kolis, J.W. Revisiting the hydrothermal growth of YAG. J. Cryst. Growth 2012, 356, 58-64. [CrossRef]

119. Moore, C.A.; Brown, D.C.; Sanjeewa, L.D.; McMillen, C.D.; Kolis, J.W. Yb:Lu $\mathrm{O}_{3}$ hydrothermally-grown single-crystal and ceramic absorption spectra obtained between 298 and 80 K. J. Lumin. accepted for publication. 2015. 
120. Brown, D.C.; Kowalewski, K.; Envid, V.; Zembeck, J.; Kolis, J.W.; McMillen, C.D.; Geisber, H. Advanced smart multi-functional laser crystals for next generation solid-state lasers. In Technology For Defense and Security VIII (Proceedings of SPIE); Dubinskii, M., Post, S., Eds.; SPIE Press: Bellingham, MA, USA, 2014. [CrossRef]

121. Sträßer, A.; Ostermeyer, M. Improving the brightness of side pumped power amplifiers by using core doped ceramic rods. Opt. Express 2006, 14, 6687-6693. [CrossRef]

122. Lucianetti, A.; Weber, R.; Hodel, W.; Weber, H.P.; Papashvili, A.; Konyushkin, V.A.; Basiev, T.T. Beam-quality improvement pf a passively Q-switched Nd:YAG laser with a core-doped rod. Appl. Opt. 1999, 38, 1777-1783. [CrossRef] [PubMed]

123. Tsunekane, M.; Taira, T. Diode edge-pumped, composite ceramic Nd:YAG/Sm:YAG microchip lasers. In Proceedings of the Frontiers in Optics 2010, Rochester, NY, USA, 2010.

124. Bowman, S.R.; O'Connor, S.; Condon, N.J. Diode pumped yellow dysprosium laser. Opt. Express 2012, 20, 12906-12911. [CrossRef] [PubMed]

125. Yagi, H.; Bisson, J.F.; Ueda, K.; Yanagitani, T. $\mathrm{Y}_{3} \mathrm{Al}_{5} \mathrm{O}_{12}$ ceramic absorbers for the suppression of parasitic oscillations in high-power Nd:YAG lasers. J. Lumin. 2006, 121, 88-94. [CrossRef]

126. Ripin, D.J.; Ochoa, J.R.; Aggarwal, R.L.; Fan, T.Y. 165-W cryogenically cooled Yb:YAG laser. Opt. Lett. 2004, 29, 2154-2156. [CrossRef] [PubMed]

127. Ripin, D.J.; Ochoa, J.R.; Aggarwal, R.L.; Fan, T.Y. 300-W cryogenically cooled Yb:YAG laser. IEEE J. Quantum Electron. 2005, 41, 1274-1277. [CrossRef]

128. Tokita, S.; Kawanaka, J.; Fujita, M.; Kawashima, T.; Izawa, Y. Sapphire-conductive end-cooling of high power cryogenic Yb:YAG lasers. Appl. Phys. B Lasers Opt. 2005, 80, 635-638. [CrossRef]

129. Brown, D.C.; Singley, J.M.; Yager, E.; Kuper, J.W.; Lotito, B.J.; Bennett, L.L. Innovative high-power CW Yb:YAG laser. In Proceedings of SPIE 6552, Laser Source Technology for Defense and Security III, Orlando, FL, USA, 9 April 2007. [CrossRef]

130. Brown, D.C.; Singley, J.M.; Yager, E.; Kowalewski, K.; Guelzow, J.; Kuper, J.W. Kilowatt class high-power CW Yb:YAG laser. In Proceedings of SPIE 6952, Laser Source Technology for Defense and Security IV, Orlando, FL, USA, 16 March 2008. [CrossRef]

131. Furuse, H.; Kawanake, J.; Takeshita, K.; Miyanaga, N.; Saika, T.; Imasaki, K. Total-reflection active-mirror laser with cryogenic Yb:YAG ceramics. Opt. Lett. 2009, 34, 3439-3441. [CrossRef] [PubMed]

132. Brown, D.C.; Singley, J.M.; Kowalewski, K.; Guelzow, J.; Vitali, V. High sustained average power cw and ultrafast Yb:YAG near-diffraction-limited cryogenic solid-state laser. Opt. Express 2010, 18, 24770-24792. [CrossRef] [PubMed]

133. Furuse, H.; Kawanaka, J.; Miyanga, N.; Saiki, T.; Imasaki, K.; Fujita, M.; Takeshita, K.; Ishii, S.; Izawa, Y. Zig-zag active-mirror laser with cryogenic $\mathrm{Yb}^{3+}$ :YAG/YAG composite ceramics. Opt. Express 2011, 19, 2448-2455. [CrossRef] [PubMed]

134. Ricaud, S.; Papapdopoulos, D.N.; Pelligrina, A.; Balembois, F.; Georges, P.; Courjaud, A.; Camy, P.; Doualan, J.L.; Moncorgé, R.; Druon, F. High-power diode-pumped cryogenically cooled $\mathrm{Yb}: \mathrm{CaF}_{2}$ laser with extremely low quantum defect. Opt. Lett. 2011, 36, 1602-1604. [CrossRef] [PubMed]

135. Feve, J.P.M.; Shortoff, K.E.; Bohn, M.J.; Brasseur, J.K. High average power diamond Raman laser. Opt. Express 2011, 19, 913-922. [CrossRef] [PubMed]

136. Vretenar, N.; Newell, T.C.; Carson, T.; Lucas, T.; Latham, W.P.; Bostanci, H.; Lindauer, J.J.; Saarloos, B.A.; Rini, D.P. Cryogenic ceramic 277 watt Yb:YAG thin-disk laser. Opt. Eng. 2012. [CrossRef]

137. Ganija, M.; Ottaway, D.; Veitch, P.; Munch, J. Cryogenic, high power, near diffraction limited, Yb:YAG slab laser. Opt. Express 2013, 21, 6973-6978. [CrossRef] [PubMed]

138. Fan, T.Y.; Ripin, D.J.; Hybl, J.D.; Gopinath, J.T.; Goyal, A.K.; Rand, D.A.; Augst, S.J.; Ochoa, J.R. Cryogenically Cooled Solid-State Lasers. Recent Developments and Future Prospects; MIT Lincoln Laboratory Internal Presentation: Lexington, MA, USA, 2010.

139. Kärtner, F.X. Laser Technology for Compact X-ray Sources. Doha CLS-Workshop Presentation. 2014.

140. Mackenzie, J.I.; Bailey, W.O.S.; Kim, J.W.; Pearson, L.; Shen, D.Y.; Yang, Y.; Clarkson, W.A. Tm:fiber laser in-band pumping a cryogenically-cooled Ho:YAG laser. In Solid State Lasers XVIII: Technology and Devices (Proceedings of SPIE); Clarkson, W.A., Hodges, N., Shori, R.K., Eds.; Bellingham, MA, USA, 2009. [CrossRef]

141. Ter-Gabrielyan, N.; Merkle, L.D.; Newburgh, G.A.; Dubinskii, M. Resonantly-pumped $\mathrm{Er}^{3+}: \mathrm{Y}_{2} \mathrm{O}_{3}$ ceramic laser for remote $\mathrm{CO}_{2}$ monitoring. Laser Phys. 2009, 19, 867-869. [CrossRef] 
142. Sunamyan, T.; Kanskar, M.; Xiao, Y.; Kedlaya, D.; Dubinskii, M. High power diode-pumped 2.7- $\mu \mathrm{m} \operatorname{Er}^{3+}: \mathrm{Y}_{2} \mathrm{O}_{3}$ laser with nearly quantum defect-limited efficiency. Opt. Express 2011, 19, A1082-A1087. [CrossRef] [PubMed]

143. Ter-Gabrielyan, N.; Merkle, L.D.; Ikesue, A.; Dubinsii, M. Ultralow quantum-defect eye-safe Er:Sc${ }_{2} \mathrm{O}_{3}$ laser. Opt. Lett. 2008, 33, 1524-1526. [CrossRef] [PubMed]

144. Ter-Gabrielyan, N.; Dubinskii, M.; Newburgh, G.A.; Michael, A.; Merkle, L.D. Temperature dependence of a diode-pumped cryogenic Er:YAG laser. Opt. Express 2009, 17, 7159-7169. [CrossRef] [PubMed]

145. Ter-Gabrielyan, N.; Fromzel, V.; Merkle, L.D.; Dubinskii, M. Resonant in-band pumping of cryo-cooled $\mathrm{Er}^{3+}$ :YAG laser at 1532, 1534, and $1546 \mathrm{~nm}$ : a comparative study. Opt. Mater. Express 2011, 1, 223-233. [CrossRef]

146. Seltzer, S.D.; Shaw, M.J.; Kukla, M.J.; Unternahrer, J.R.; Dinndorf, K.M.; Beattie, J.A.; Chickless, E.P. A 400 W cryogenic Er:YAG slab laser at 1645 nm. In Proceedings of SPIE 7686, Laser Technology for Defense and Security VI, Orlando, FL, USA, 5 April 2010. [CrossRef]

147. Kawanaka, J.; Takeuchi, Y.; Yoshida, A.; Pearce, S.J.; Yasuhara, R.; Kawashima, T.; Kan, H. Highly efficient cryogenically-cooled Yb:YAG laser. Laser Phys. 2010, 20, 1079-1084. [CrossRef]

148. Tokita, S.; Kawanaka, J.; Fujita, M.; Kawashima, T.; Izawa, Y. Efficient high-average-power operation of a Q-switched cryogenic Yb:YAG laser oscillator. Jpn. J. Appl. Phys. 2005, 44, L1520-L1531. [CrossRef]

149. Perevezentsev, E.A.; Mukhin, I.B.; Kuznetsov, I.I.; Palashov, O.V.; Khazanov, E.A. Cryogenic disk Yb:YAG laser with $120 \mathrm{~mJ}$ energy at $500 \mathrm{~Hz}$ repetition rate. Quantum Electron. 2013, 43, 207-210. [CrossRef]

150. Manni, J.G.; Hybl, J.D.; Rand, D.; Ripin, D.J.; Ochoa, J.R.; Fan, T.Y. 100-W Q-switched cryogenically cooled Yb:YAG laser. IEEE J. Quantum Electron. 2010, 46, 95-98. [CrossRef]

151. Cheng, X.; Wang, J.; Yang, Z.; Liu, J.; Li, L.; Shi, X.; Huang, W.; Wang, J.; Chen, W. A high energy nanosecond cryogenic cooled Yb:YAG active-mirror amplifier system. High Power Laser Sci. Eng. 2014, 2, 1-4. [CrossRef]

152. Mason, P.D.; Banerjee, S.; Ertel, K.; Phillips, P.J.; Greenhalgh, J.; Collier, J.L. DiPOLE-An efficirnt and scalable high pulse energy and high average power cryogenic gas cooled multi-slab amplifier concept. Plasma Fusion Res. 2013, 8, 1-4. [CrossRef]

153. Kawanaka, J.; Yamakawa, K.; Nishioka, H.; Ueda, K. 30-mJ, diode-pumped chirped-pulse Yb:YLF regenerative amplifier. Opt. Lett. 2003, 28, 2121-2123. [CrossRef] [PubMed]

154. Bayramian, A.; Armstrong, P.; Ault, E.; Beach, R.; Bibeau, C.; Caird, J.; Campbell, R.; Chai, B.; Dawson, J.; Ebbers, C.; et al. The Mercury project: A high average power gas-cooled laser for inertial fusion energy development. Fusion Sci. Technol. 2007, 52, 383-387.

155. Lucianetti, A.; Albach, D.; Chanteloup, J.C. Active-mirror-laser-amplifier thermal management with tunable helium pressure at cryogenic temperatures. Opt. Express 2011, 19, 12766-12780. [CrossRef] [PubMed]

156. Vaupel, A.; Bodnar, N.; Webb, B.; Shah, L.; Richardson, M. Concepts, performance review, and prospects of table-top, few-cycle optical parametric chirped-pulse amplification. Opt. Engin. 2014, 53, 1-12. [CrossRef]

157. Fattahi, H.; Barros, H.G.; Gorjan, M.; Nubbemeyer, T.; Alsaif, B.; Teisset, C.Y.; Schultze, M.; Prinz, S.; Haefner, M.; Ueffing, M.; et al. Third generation femtosecond technology. Optica 2014, 1, 45-63. [CrossRef]

158. Tokita, S.; Kawanaka, J.; Izawa, Y.; Fujita, M.; Kawashima, T. 23.7-W picosecond cryogenic-Yb:YAG multipass amplifier. Opt. Express 2007, 15, 3955-3961. [CrossRef] [PubMed]

159. Akahane, Y.; Aoyama, M.; Ogawa, K.; Tsuji, K.; Tokita, S.; Kawanaka, J.; Nishioka, H.; Yamakawa, K. High-energy, diode-pumped, picosecond Yb:YAG chirped-pulse regenerative amplifier for pumping chirped-pulse amplification. Opt. Lett. 2007, 32, 1899-1901. [CrossRef] [PubMed]

160. Hong, K.H.; Siddiqui, A.; Moses, J.; Gopinath, J.; Hybl, J.; Ilday, F.Ö.; Fan, T.Y.; Kärtner, F.X. Generation of $287 \mathrm{~W}, 5.5 \mathrm{ps}$ pulses at $78 \mathrm{MHz}$ repetition rate from a cryogenically cooled Yb:YAG amplifier seeded by a fiber chirped-pulse amplification system. Opt. Lett. 2008, 33, 2473-2475. [CrossRef] [PubMed]

161. Hong, K.H.; Lai, C.J.; Siddiqui, A.; Kärtner, F.X. 130-W picosecond green laser based on a frequency-doubled hybrid cryogenic Yb:YAG amplifier. Opt. Express 2009, 19, 16911-16919. [CrossRef] [PubMed]

162. Reagan, B.A.; Curtis, A.H.; Wernsing, K.A.; Furch, F.J.; Luther, B.M.; Rocca, J.J. Development of high energy diode-pumped thick-disk Yb:YAG chirped-pulse-amplification lasers. IEEE J. Quantum Electron. 2012, 48, 827-835. [CrossRef]

163. Hong, K.H.; Gopinath, J.T.; Rand, D.; Siddiqui, A.M.; Huang, S.W.; Li, E.; Eggleton, B.J.; Hybl, J.D.; Fan, T.Y.; Kärtner, F.X. High-energy, kHz-repetition-rate, ps cryogenic Yb:YAQ chirped-pulse amplifier. Opt. Lett. 2010, 35, 1752-1754. [CrossRef] [PubMed] 
164. Kowalewski, K.; Zembec, J.; Envid, V.; Brown, D.C. $201 \mathrm{~W}$ picosecond green laser using a mode-locked fiber driven cryogenic Yb:YAG amplifier system. Opt. Lett. 2012, 37, 4633-4735. [CrossRef] [PubMed]

165. Hong, K.H.; Huang, S.W.; Moses, J.; Fu, X.; Lai, C.J.; Cirmi, G.; Sell, A.; Granados, E.; Keathley, P.; Kärtner, F.X. High-energy, phase-stable, ultrabroadband $\mathrm{kHz}$ OPCPA at $2.1 \mu \mathrm{m}$ pumped by a picosecond cryogenic Yb:YAG laser. Opt. Express 2011, 19, 15538-15548. [CrossRef] [PubMed]

166. Curtis, A.H.; Reagan, B.A.; Wernsing, K.A.; Furch, F.J.; Luther, B.M.; Rocca, J.J. Demonstration of a compact $100 \mathrm{~Hz}, 0.1 \mathrm{~J}$, diode-pumped picosecond laser. Opt. Lett. 2011, 36, 2164-2166. [CrossRef] [PubMed]

167. Miller, D.E.; Zapata, L.E.; Ripin, D.J.; Fan, T.Y. Sub-picosecond pulses at $100 \mathrm{~W}$ average power from a Yb:YLF chirped-pulse amplification system. Opt. Lett. 2012, 37, 2700-2702. [CrossRef] [PubMed]

168. Petrov, V.V.; Pestryakov, E.V.; Laptev, A.V.; Petrov, V.A.; Kuptsov, G.V. Pump channel of parametric amplifoer of terawatt femtosecond $\mathrm{Yb}$ laser system. Atmos. Ocean. Opt. 2014, 27, 344-347. [CrossRef]

169. Zapata, L.E.; Lin, H.; Calendron, A.L.; Cankaya, H.; Hemmer, M.; Reichert, F.; Huang, W.R.; Granados, E.; Hong, K.H.; Kärtner, F.X. Cryogenic Yb:YAG composite-thin-disk for high energy and average power amplifiers. Opt. Lett. 2015, 40, 2610-2613. [CrossRef] [PubMed]

170. Pugžlys, A.; Andriukaitas, G.; Sidorov, D.; Irshad, A.; Baltuška, A.; Lai, W.J.; Phua, P.B.; Su, L.; Xu, J.; Li, R.; et al. Spectroscopy and lasing of cryogenically cooled $\mathrm{Yb}, \mathrm{Na}: \mathrm{CaF}_{2}$. Appl. Phys. B Lasers Opt. 2009, 97, 339-350. [CrossRef]

171. Divoky, M.; Smrz, M.; Chyla, M.; Sikocinski, P.; Novak, O.; Huynh, J.; Nagisetty, S.S.; Miura, T.; Pilař, J.; Slezak, O.; et al. Overview of the HiLASE project: High average power pulsed DPSSL systems for research and industry. High Power Laser Sci. Eng. 2014, 2, 1-10. [CrossRef]

172. Novák, O.; Miura, T.; Smrž, M.; Chyla, M.; Nagisetty, S.S.; Mužik, J.; Linnemann, J.; Turčičova, H.; Jambunathan, V.; Slezák, O.; et al. Review, Status of the high average power diode-pumped solid state laser development at HiLASE. Appl. Sci. 2015, 5, 637-665. [CrossRef]

173. Endo, A.; Sikocinski, P.; Chyla, M.; Miura, T.; Mocek, T.; Sakaue, K.; Washio, M. Cryogenically cooled 1 J, ps Yb:YAG slab laser for high-brightness laser-Compton X-ray source. In Proceedings of the IPAC, Dresden, Germany, 15-20 June 2014.

(C) 2016 by the authors; licensee MDPI, Basel, Switzerland. This article is an open access article distributed under the terms and conditions of the Creative Commons by Attribution (CC-BY) license (http://creativecommons.org/licenses/by/4.0/). 